\title{
Métodos não Tradicionais de Seleção de Variáveis em Modelos de Regressão Linear
}

\author{
Vaudeluci Maria da Silva
}

\author{
DISSERTAÇÃO APRESENTADA \\ $\mathrm{AO}$ \\ INSTITUTO DE MATEMÁTICA E ESTATÍSTICA \\ DA \\ UNIVERSIDADE DE SÃO PAULO \\ PARA \\ OBTENÇÃO DO GRAU DE MESTRE \\ EM \\ ESTATÍSTICA
}

Área de concentração: Estatística

Orientadora: Prof ${ }^{a}$ Dra. Silvia Nagib Elian

- Durante o desenvolvimento deste trabalho, a autora recebeu apoio financeiro da Capes -

- São Paulo, dezembro de 2004 - 


\section{Métodos não Tradicionais de Seleção de Variáveis em Modelos de Regressão Linear}

Este exemplar corresponde à redação final da dissertação devidamente corrigida e defendida por Vaudeluci Maria da Silva e aprovada pela comissão julgadora.

São Paulo, fevereiro de 2004.

Banca examinadora:

- Prof ${ }^{a}$ Dra. Silvia Nagib Elian (Orientadora) (IME - USP)

- Prof ${ }^{a}$ Dra. Carmen Diva Saldiva de André (IME - USP)

- Prof ${ }^{a}$ Dra. Cecília Candolo (UFSCar) 
A Deus por fornecer condições necessárias para continuar batalhando pelos meus objetivos. 


\section{AGRADECIMENTOS}

A prof ${ }^{a}$ Dra. Silvia Nagib Elian, pela orientação, profissionalismo, amizade, paciência e auxílio dado em todos os momentos de dificuldade.

À minha família pela paciência, apoio e compreensão pelas minhas ausências.

Ao Rogério, meu esposo e companheiro, pelos conselhos e incentivo nos momentos mais difíceis.

À família do meu esposo, por acreditar em minha capacidade e me fornecer apoio em todos os momentos.

Ao Instituto de Matemática e Estatística, em especial ao pessoal da biblioteca e da secretaria da pós-graduação.

Ao Ailton Augusto (Feijão), da secretaria da pós-graduação, por seus esclarecimentos e ajuda dada no início do mestrado.

À Prof ${ }^{a}$ Dra. Sônia Regina L. Garcia, pelo auxílio na interpretação dos resultados geométricos.

Ao meu colega João, pela importante ajuda no trabalho de programação.

Aos Prof ${ }^{\circ}$ s. Galvão, Rinaldo, Carlos Dantas, Gilberto, Antônio Carlos, Luís Gustavo, Vladimir, Wagner, Sérgio, Heleno Bolfarine, e as Prof ${ }^{\mathrm{a}} \mathrm{s}$ Silvia Ferrari, Elisabeti, Lúcia, Elisete, Márcia e Cláudia pelo apoio e pela contribuição para o crescimento do meu conhecimento. 
Aos Prof ${ }^{\circ}$ s do departamento de Licenciatura em Matemática que contribuíram com parte da minha formação.

Aos colegas da pós: Sueli, Willian, Domingo, Cléber, Maíra, Elias, Paulo Tadeu, Juvêncio e Caio pela amizade, contribuição e incentivo.

Aos meus amigos incondicionais: Elaine, Cristiane (Tiani), Christina Mirabal, Antônio Salomão, Thiago Rodrigo, Patrícia Alexandrino, Flanvilma, Vanessa, Rosângela e Ângela que mesmo quando estiveram longe, sempre foram especiais.

Ao meu pai Francisco e meu irmão Josenildo, ambos já falecidos, pelo grande incentivo dado para eu nunca desistir dos meus objetivos, e pelo grande apoio no início da minha jornada.

Finalmente, a todos que de alguma forma contribuíram para a realização deste trabalho. 


\section{RESUMO}

Neste trabalho, apresentamos uma descrição dos métodos não tradicionais de seleção de variáveis preditoras no modelo de regressão linear. Inicialmente, fizemos um breve levantamento dos métodos tradicionais com o objetivo de comparação futura. Posteriormente, foram apresentados métodos do tipo redução, que restringem as estimativas de mínimos quadrados usuais. Numa etapa seguinte, estudamos métodos com enfoque Bayesiano na seleção de variáveis preditoras. Finalizando, aplicamos o procedimento de seleção Lasso e um dos procedimentos Bayesianos a um conjunto de dados presente na literatura. 


\section{ABSTRACT}

In this dissertation, we describe unusual procedures of variable selection in the linear regression models. First, we present a review of the popular methods of variable selection. Further, we describe shrinkage methods applied in the least squares estimators that can be used to select predictor variables. The last part of the work is devoted to Bayesian methods of variables selection. Finally, we apply the Lasso and one of the Bayesian procedures to a data set well known in the variables selection literature. 


\section{SUMÁRIO}

1 INTRODUÇÃO

2 MÉTODOS TRADICIONAIS DE SELEÇÃO DE VARIÁVEIS PREDITORAS 3

2.1 Introdução . . . . . . . . . . . . . . . . . . . . 3

2.2 Todas as possíveis regressões $\ldots \ldots \ldots \ldots \ldots \ldots$

2.3 Melhor subconjunto de regressões . . . . . . . . . . . . . . . 9

2.4 Seleção Forward . . . . . . . . . . . . . . . . . . . . . . . 9

2.5 Eliminação Backward . . . . . . . . . . . . . . . . . . . . 11

2.6 Stepwise . . . . . . . . . . . . . . . . . . . . 13

2.7 Press. . . . . . . . . . . . . . . . . . . . . . 14

2.8 Ridge Regression . . . . . . . . . . . . . . . . . . . . . 15

2.9 Considerações finais . . . . . . . . . . . . . . . . . . . . . 19

3 MÉTODOS DE SELEÇÃo DE VARIÁVEIS DO TIPO REDUÇÃo 21

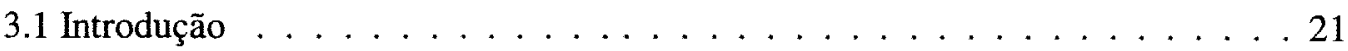

3.2 Garrote não Negativo. . . . . . . . . . . . . . . . . . . 22

3.2.1 Introdução e conceitos básicos. . . . . . . . . . . . . . 22

3.2.2 Seleção de modelos através do método Garrote não Negativo . . . . . 30

3.3 Lasso . . . . . . . . . . . . . . . . . . . . . . . . . . . . . . . . 41

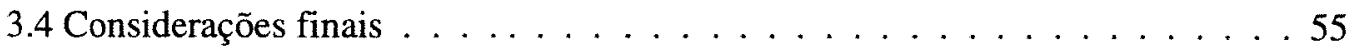

4 MÉTODOS DE SELEÇÃO DE VARIÁVEIS COM ENFOQUE BAYESIANO 57

4.1 Introdução . . . . . . . . . . . . . . . . . . . . . . 57

4.2 Método Bayesiano. . . . . . . . . . . . . . . . . . 58

4.3 Densidade Preditiva Bayesiana . . . . . . . . . . . . . . . . 75

4.4 Amostrador de Gibbs . . . . . . . . . . . . . . . . . . . . . . . 82

4.5 Considerações finais . . . . . . . . . . . . . . . . . . 99 
5 ANÁLISE DO CONJUNTO DE DADOS DE HALD 100

5.1 Introdução . . . . . . . . . . . . . . . . . . . . . . 100

5.2 Método Bayesiano . . . . . . . . . . . . . . . . . . . . . . 102

5.3 Lasso . . . . . . . . . . . . . . . . . . . . . . . . . . . . 105

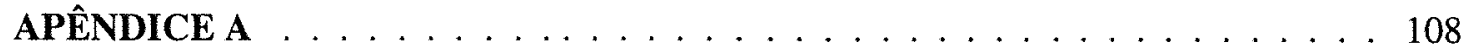

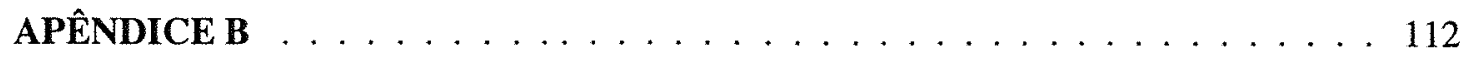

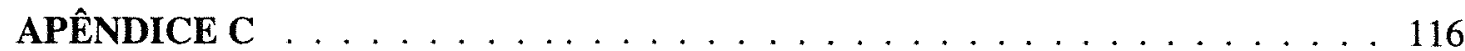

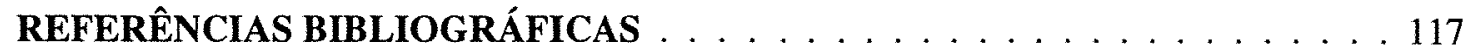




\section{Capítulo 1}

\section{INTRODUÇÃO}

Um problema importante na construção e análise de modelos de regressão linear múltipla é a seleção de variáveis preditoras ou explicativas a serem incluídas.

Procedimentos de seleção são úteis por várias razões. É conhecido o fato de que a adição de uma nova variável explicativa ao modelo aumenta a variância do previsor obtido através da equação de regressão. Além disso, modelos com menos variáveis explicativas são mais simples. Uma outra vantagem é determinar quais variáveis explicativas são importantes na previsão da variável resposta. Quando o número de variáveis preditoras utilizadas é grande, pode surgir instabilidade no ajuste, devido a uma possível multicolinearidade.

Inúmeros métodos de seleção de variáveis podem ser encontrados na literatura. Métodos tradicionais como Todas as Possíveis Regressões, Melhor Subconjunto de Regressões, Forward, Backward, Stepwise, Press e Ridge Regression são apresentados e amplamente analisados em livros de Regressão (Montgomery e Peck (1992), Neter, Kutner, Nachtsheim e Wasserman (1996), 
Draper e Smith (1998)). A maioria destes métodos está implementada nos pacotes computacionais como SPSS, BMDP, Minitab, SAS e S-Plus.

O objetivo deste trabalho é fazer um levantamento e estudar detalhadamente os métodos de seleção de variáveis não tradicionais e que de um modo geral não são apresentados nos livros clássicos de Análise de Regressão.

No Capítulo 2 da dissertação, é apresentada uma breve descrição dos métodos tradicionais de seleção de variáveis preditoras nos modelos de regressão. Abordaremos os métodos de seleção do tipo redução no Capítulo 3. No Capítulo 4, trataremos de métodos de seleção de variáveis com enfoque Bayesiano. Alguns dos métodos não tradicionais serão aplicados no conjunto de dados de Hald (Draper e Smith, 1998) com o propósito de compará-los com os métodos tradicionais e verificar se selecionavam as mesmas variáveis. Os resultados das aplicações dos métodos e os procedimentos para os programas serão exibidos no Capítulo 5. 


\section{Capítulo 2}

\section{MÉTODOS TRADICIONAIS DE SELEÇÃO DE VARIÁVEIS PREDITORAS}

\subsection{Introdução}

Neste capítulo, serão discutidos vários métodos tradicionais de seleção de variáveis preditoras em regressão linear. Todos os métodos que serão apresentados seguem o princípio: dada a variável dependente y e um conjunto de possíveis variáveis preditoras $\mathrm{x}_{1}, \mathrm{x}_{2}, \ldots, \mathrm{x}_{\mathrm{r}}$, o problema consiste em determinar o "melhor" modelo da forma $\mathrm{y}=\beta_{0}+z_{1} \beta_{1}+\cdots+z_{q} \beta_{q}+\varepsilon$, sendo que $\beta_{0}, \beta_{1}, \ldots, \beta_{q}$ são parâmetros desconhecidos; $\varepsilon$ é variável aleatória, e $z_{1}, \ldots, z_{q}$ são variáveis preditoras, funções de uma ou mais das variáveis $x_{k}, \mathrm{k}=1,2, \ldots, \mathrm{r}$. Observe que quando não há transformação, $z_{k}=x_{k}, \mathrm{k}=1,2, \ldots, \mathrm{q}, \mathrm{e} q=r$.

Seja $y_{i}$ uma observação extraída aleatoriamente da população cujos valores das variáveis preditoras são $z_{1}, z_{2}, \ldots, z_{q}, i=1,2, \ldots, n$, com $n$ correspondendo ao 
tamanho da amostra. Em todo o estudo, admitiremos que o valor previsto da observação $y$ (dado as variáveis preditoras $z_{1}, z_{2}, \ldots, z_{q}$ ) é

$$
\hat{y}=\hat{\beta}_{0}+z_{1} \hat{\beta}_{1}+\ldots+z_{q} \hat{\beta}_{q}
$$

sendo que $\hat{\beta}_{0}, \hat{\beta}_{1}, \ldots, \hat{\beta}_{q}$ são estimadores de $\beta_{0}, \beta_{1}, \ldots, \beta_{q}$.

As somas de quadrados total, do resíduo e da regressão, respectivamente, são definidas da forma usual como

$$
S Q T=\sum_{i}\left(y_{i}-\bar{y}\right)^{2}, S Q \operatorname{Re} s=\sum_{i}\left(y_{i}-\hat{y}_{i}\right)^{2} \text { e } S Q \operatorname{Re} g=\sum_{i}\left(\hat{y}_{i}-\bar{y}\right)^{2},
$$

sendo que $\bar{y}$ é a média amostral da variável $y$ e $\hat{y}_{i}$ é o valor previsto de $y_{i}$, $\mathrm{i}=1,2, \ldots, \mathrm{n}$.

Definidos esses elementos, descreveremos a seguir os principais métodos de seleção.

\subsection{Todas as possíveis regressões}

Este método consiste em ajustar todas as possíveis equações de regressão linear de $y$ em subconjuntos de $z_{1}, \ldots, z_{q}$. Assumindo que o parâmetro intercepto $\beta_{0}$ e uma variável dummy $z_{0}=1$ a ele associada estejam incluídos em todas equações, então se há $q$ variáveis preditoras, há ao todo $2^{q}$ possíveis equações de regressão a serem estimadas e examinadas. Se $q=10$, um número não extraordinariamente excessivo, $2^{10}=1024$ equações de regressão seriam examinadas, o que tornaria inviável o uso da estratégia de investigar todas as equações possíveis. Feito o ajuste, 
cada equação é avaliada segundo algum critério. Os três critérios mais usados segundo Draper e Smith (1998) são:

1. Coeficiente de determinação múltipla $\left(R_{p}^{2}\right)$

2. Quadrado médio do resíduo ( $\mathrm{QMRes}_{\mathrm{p}}$ )

3. Estatística $C_{p}$ de Mallows $(1973,1995)$.

O número de parâmetros na equação de regressão está subscrito. Nos três critérios há $p$ parâmetros envolvidos, se $\mathrm{p}=\mathrm{q}+1$, temos $p-1=q$ parâmetros associados às variáveis explicativas e um parâmetro correspondendo ao intercepto $\beta_{0}$.

Uso do coeficiente de determinação múltipla $R_{p}^{2}$ ou $\mathrm{SQRes}_{\mathrm{p}}$

A estatística $R_{p}^{2}$ representa o coeficiente de determinação múltipla ou coeficiente de explicação para equações de regressão com $p$ parâmetros. O coeficiente é usado para medir a qualidade do ajuste de um modelo e é definido por

$$
R_{p}^{2}=\frac{S Q \operatorname{Re} g_{p}}{S Q T}=1-\frac{S Q \operatorname{Re} s_{p}}{S Q T}
$$

sendo que $S Q \operatorname{Re} s_{p}$ e $S Q T$ indicam a soma de quadrados do resíduo e a soma de quadrados total, respectivamente. Como o denominador é constante para todas as possíveis equações de regressão, $R_{p}^{2}$ varia inversamente com SQRes ${ }_{\mathrm{p}}$.

O intervalo de possíveis valores de $R_{p}^{2}$ é [0,1], sendo que $R_{p}^{2}=1$ corresponde ao ajuste perfeito. As equações com valores altos de $R_{p}^{2}$ são as preferidas. A 
estatística $R_{p}^{2}$ e a estatística soma de quadrados do resíduo SQRes ${ }_{\mathrm{p}}$ fornecem informações equivalentes como vimos em (2.1). Os subconjuntos com menor SQRes ${ }_{\mathrm{p}}$ são considerados "bons", pois $R_{p}^{2}$ se aproxima de 1 .

Observe no entanto que não é desejado identificar os subconjuntos que maximizem a estatística $R_{p}^{2}$. Isto porque $R_{p}^{2}$ em geral aumenta quando $p$ aumenta e o máximo de $R_{p}^{2}$ será quando todas as variáveis preditoras, $q-1$, são incluídas na equação de regressão. A intenção é usar a estatística $R_{p}^{2}$ para encontrar o ponto a partir do qual uma variável preditora a mais não seria útil na equação, visto que conduz a um aumento em $R_{p}^{2}$ muito pequeno. De acordo com Draper e Smith (1998), é adequado também examinar os pares de correlações entre $z_{k}$ e $z_{k^{*}}$ e entre $z_{k}$ e $y$, sendo que $k, k^{*}=1, \ldots, q$ e $k \neq k^{*}$, antes de tomar a decisão. Quando existem pares de variáveis $\left(z_{k}, z_{k}\right.$ ) que são altamente correlacionados, então basta uma dessas variáveis para explicar a variável resposta. A variável escolhida será aquela que for mais correlacionada com a variável resposta. Entretanto, a escolha da "melhor" equação de regressão ficará a critério do analista da área.

\section{Uso do quadrado médio do resíduo QMRes $_{\mathrm{p}}$ ou $\bar{R}_{p}^{2}$}

A estatística QMRes $_{\mathrm{p}}$ (quadrado médio do resíduo) e a estatística $\bar{R}_{p}^{2}$ (coeficiente de determinação múltipla ajustado pelos graus de liberdade ou coeficiente de explicação ajustado) são definidos respectivamente por

$$
Q M \operatorname{Re} s_{p}=\frac{S Q \operatorname{Re} s_{p}}{n-p} \quad \text { e } \quad \bar{R}_{p}^{2}=1-\left(\frac{n-1}{n-p}\right)\left(1-R_{p}^{2}\right) .
$$


A equivalência dos critérios QMRes $_{\mathrm{p}}$ e $\bar{R}_{p}^{2}$ segue de

$$
\bar{R}_{p}^{2}=1-\left(\frac{n-1}{n-p}\right)\left(1-R_{p}^{2}\right)=1-\left(\frac{n-1}{n-p}\right) \frac{S Q \operatorname{Re} s_{p}}{S Q T}=1-\frac{Q M \operatorname{Re} s_{p}}{\frac{S Q T}{n-1}} .
$$

O valor de $\bar{R}_{p}^{2}$ cresce se e somente se QMRes $_{\mathrm{p}}$ decresce, já que $S Q T /(n-1)$ é constante. Logo, a equação de regressão que minimiza $\mathrm{QMRes}_{\mathrm{p}}$ também maximiza $\bar{R}_{p}^{2}$.

Em geral, observa-se que inicialmente QMRes $_{\mathrm{p}}$ decresce à medida que variáveis preditoras são incluídas na equação. Quando são acrescentadas variáveis desnecessárias, o valor de QMRes ${ }_{p}$ tende a estabilizar e aproximar-se do verdadeiro valor de $\sigma^{2}$, visto que QMRes ${ }_{\mathrm{p}}$ é um estimador não viciado de $\sigma^{2}$. O objetivo é encontrar o número de parâmetros para o qual isso ocorre e que a equação apresente o menor valor do quadrado médio do resíduo. Uma maneira de obter a "melhor" equação é primeiramente obter as médias dos valores dos $\operatorname{QMRes}_{\mathrm{p}},\left(\overline{Q M \operatorname{Re} s_{p}}\right)$, para as equações com o mesmo número de parâmetros $(p)$. Em seguida, devemos observar em torno de qual valor $\overline{Q M \operatorname{Re} s_{p}}$ se estabiliza, tal valor seria considerado o melhor $\mathrm{QMRes}_{\mathrm{p}}$. Finalmente, determina-se dentre todas as equações que apresentem QMRes $_{\mathrm{p}} \cong\left(\overline{Q M \operatorname{Re} s_{p}}\right.$ estabilizado $)$ a que tenha o menor $p$, isto é, o menor número de variáveis preditoras $(p-1)$.

Uso da estatística $C_{p}$ de Mallows 
A estatística $C_{p}$ associada a uma particular equação com parâmetros é definida como

$$
C_{p}=\frac{S Q \operatorname{Re} s_{p}}{Q M \operatorname{Re} s_{p}^{*}}-(n-2 p),
$$

sendo que $S Q R e s_{p}$ é a soma de quadrados de resíduo desta equação, $n$ é o tamanho da amostra e QMRes* é o quadrado médio do resíduo da equação contendo todas as variáveis preditoras. É presumido que $\mathrm{QMRes}_{\mathrm{p}}^{*}$ é o estimador não viciado de $\sigma^{2}$, isto é, $E\left(Q M \operatorname{Re} s_{p}^{*}\right)=\sigma^{2}$.

Para cada $p$, há vários valores da estatística $C_{p}$ relativos às equações com $p$ parâmetros, exceto quando todas as variáveis preditoras estão incluídas. Se uma equação com parâmetros é adequada, isto é, não existe falta de ajuste, então $E\left(S Q \operatorname{Re} s_{P}\right)=(n-p) \sigma^{2}$ e assim, o valor esperado de $C_{p}$ deve ser aproximadamente $p$.

A "melhor" equação é escolhida depois de se inspecionar o gráfico de $C_{p}$ em função de $p$ e examinar os pontos, representantes das equações, que estão bastante perto da linha $C_{p}=p$. Observe que para a equação completa com todas as variáveis preditoras $C_{p}=p$.

Equações com viés ou com falta de ajuste dão origem a pontos acima da linha $C_{p}=p$, ou seja, apresentam $C_{p}>p$. As equações bem ajustadas podem ter $C_{p}<p$. Assim sendo, devemos procurar uma equação de regressão com um valor $C_{p}$ baixo mais ou menos igual a $p$ e com o mínimo possível de variáveis preditoras. 


\subsection{Melhor subconjunto de regressões}

Este método determina as $M$ melhores equações de regressão, sendo que $M$ é fixado a priori, segundo qualquer um dos critérios discutidos na Seção 2.2. Outra maneira é escolher as $M$ melhores equações dentre as equações com apenas uma variável preditora, com duas, com três e assim por diante. Segundo Draper e Smith (1998), há algumas desvantagens nesse método:

1. Tende a fornecer equações com muitas variáveis preditoras incluídas;

2. Se o valor de $M$ escolhido for pequeno, então é possível que a "melhor" equação não apareça entre as $M$ melhores estabelecidas;

3. Apresenta uma saída reduzida, contendo apenas os valores de $R^{2}, \bar{R}^{2}, C_{p}$ e $\sqrt{Q M \operatorname{Re} s}$. Este fato, no entanto, pode ser considerado uma limitação do programa e não do método.

Do ponto de vista computacional, este procedimento pode ser útil quando queremos uma rápida alternativa ao método "Todas as Possíveis Regressões", apenas definindo o valor de $M$ desejado.

\subsection{Seleção Forward}

Este método parte de um modelo em que não há variáveis preditoras e há apenas o parâmetro intercepto. Calcula-se primeiramente os coeficientes de correlação de cada variável preditora com a variável resposta $y$. A primeira variável a ser incluída na equação será a que apresentar o maior valor desta correlação em módulo. Vamos supor que seja $z_{1}$ a variável preditora mais correlacionada com $y, \mathrm{o}$ 
que implica que esta variável também produz o maior valor da estatística $F$ para testar a significância de cada variável preditora. Esta estatística permite testarmos a hipótese $H_{0}: \beta_{1}=0$ contra $H_{a}: \beta_{1} \neq 0$, sendo que $\beta_{1}$ é o coeficiente de regressão associado à variável $Z_{1}$, cuja significância desejamos avaliar. Rejeitaremos $H_{0}$ ao nível $\alpha$ se a estatística $F$ exceder o valor $F_{I N}=F_{(1-\alpha ; 1, n-2)}$ ( $F$ de entrada), sendo que $\alpha$ será o nível de significância, 1 e $n-2$ serão os números de graus de liberdade do numerador e denominador, respectivamente. Admitindo que a estatística $F$ associada à variável $z_{1}$ exceda o valor $F_{I N}$, então a variável é inserida na equação. Desta maneira, obtemos a equação de regressão $\hat{y}=\hat{\beta}_{0}+\hat{\beta}_{1} z_{1}$. Se concluirmos pela não significância de $z_{1}$, tal variável não será introduzida, o procedimento de seleção pára, tendo como equação selecionada $\hat{y}=\bar{y}$.

A segunda variável a entrar na equação é selecionada de forma similar à primeira, mas agora devemos verificar dentre as variáveis preditoras restantes, qual delas apresenta maior módulo do coeficiente de correlação parcial com y dado que a primeira variável entrou no modelo. Vamos supor que a variável com a maior correlação parcial com $y$ seja $z_{2}$, o que implica que a maior estatística $F$ parcial é

$$
F=\frac{S Q \operatorname{Re} g\left(z_{2} / z_{1}\right)}{Q M \operatorname{Re} s\left(z_{1}, z_{2}\right)}
$$

sendo que $S Q \operatorname{Re} g\left(z_{2} / z_{1}\right)=S Q \operatorname{Re} g\left(z_{1}, z_{2}\right)-S Q \operatorname{Re} g\left(z_{1}\right)$ mede o ganho com a introdução da variável $z_{2}$ no modelo com a variável $z_{1}$ já inserida e $\operatorname{QMRes}\left(\mathrm{z}_{1}, \mathrm{z}_{2}\right)$ é o quadrado médio do resíduo do modelo com as variáveis preditoras $z_{1}$ e $z_{2}$.

Se este valor $F$ parcial exceder $F_{I N}$, então $z_{2}$ é adicionada na equação. Consequentemente, obtemos uma equação de $y$ em função de $z_{1}$ e $z_{2}$, $\hat{y}=\hat{\beta}_{0}+\hat{\beta}_{1} z_{1}+\hat{\beta}_{2} z_{2}$. O processo continua de forma similar para as variáveis 
restantes. Em geral, a cada passo, a variável que tem a mais alta correlação parcial com $y$, ou equivalentemente a maior estatística $F$ parcial dado que as outras variáveis já se encontram na equação, é adicionada se a estatística $F$ parcial exceder $F_{I N}$. O processo termina quando a estatística $F$ parcial associada a cada variável indicada a ser introduzida em um particular passo não exceder $F_{I N}$, caso em que a última variável preditora é acrescentada à equação.

Este método tem a desvantagem de, após a entrada de uma nova variável, não verificar a significância das variáveis que já estavam na equação, análise esta que é feita no método Stepwise. Segundo Draper e Smith (1998), o método Seleção Forward só é recomendado se não desejamos remover nenhuma variável, a cada inclusão de variáveis na equação.

\subsection{Eliminação Backward}

Este procedimento parte de uma equação com todas as variáveis preditoras e o parâmetro intercepto e age no sentido oposto ao método de Seleção Forward. Primeiramente, analisa-se todos os valores da estatística $F$ parcial associada a cada uma das variáveis $\mathrm{z}_{1}, \mathrm{z}_{2}, \ldots, \mathrm{z}_{\mathrm{q}}$. A variável com o menor valor da estatística $F$ parcial para testar sua significância é a candidata a ser eliminada da equação. Vamos supor que a variável $z_{1}$ esteja nestas condições. Isto implica que o menor valor da estatística $F$ parcial é

$$
F=\frac{S Q \operatorname{Re} g\left(z_{1} / z_{2}, \ldots, z_{q}\right)}{Q M \operatorname{Re} s\left(z_{1}, \ldots, z_{q}\right)}
$$


sendo

que

$S Q \operatorname{Re} g\left(z_{1} / z_{2}, \ldots, z_{q}\right)=S Q \operatorname{Re} g\left(z_{1}, \ldots, z_{q}\right)-S Q \operatorname{Re} g\left(z_{2}, \ldots, z_{q}\right)$

e

$\mathrm{QMRes}\left(\mathrm{z}_{1}, \ldots, \mathrm{z}_{\mathrm{q}}\right)$ é o quadrado médio do resíduo para o modelo com as variáveis preditoras $\mathrm{z}_{1}, \ldots, \mathrm{z}_{\mathrm{q}}$.

Esta estatística permite testarmos a hipótese $H_{0}: \beta_{1}=0$ contra $H_{a}: \beta_{1} \neq 0$. Rejeitaremos $H_{0}$ ao nível $\alpha$ se a estatística $F$ parcial for menor que o valor $F_{\text {oUT }}=F_{(1-\alpha ; 1, n-2)}(F$ de saída), sendo que $\alpha$ é o nível de significância, 1 e $n-2$ são os números de graus de liberdade do numerador e denominador, respectivamente. Se a estatística $F$ parcial associada à variável $z_{1}$ for menor que o valor $F_{\text {ovt }}$, então a variável será eliminada da equação. Consequentemente, obtemos uma equação de $y$ em função das variáveis $\mathrm{z}_{2}, \ldots, \mathrm{z}_{\mathrm{q}}, \hat{y}=\hat{\beta}_{0}+\hat{\beta}_{2} z_{2}+\cdots+\hat{\beta}_{q} z_{q}$. Caso contrário, o procedimento pára e nenhuma variável sai.

Na etapa seguinte, são calculados os $q-1$ valores das estatísticas $F$ parcial das variáveis restantes, $z_{2}, z_{3}, \ldots, z_{q}$, dado que as demais, com exceção de $z_{1}$, estão no modelo. Supondo que $z_{2}$ é a variável associada à menor estatística $F$ parcial após a eliminação da variável $z_{1}$, se este valor for menor que $F_{\text {out }}$, então $z_{2}$ é eliminada da equação. Consequentemente, obtemos uma equação de $y$ em função de $z_{3}, \ldots, z_{q}$, $\hat{y}=\hat{\beta}_{0}+\hat{\beta}_{3} z_{3}+\cdots+\hat{\beta}_{q} z_{q}$ e o processo continua de forma similar para as demais variáveis. Entretanto, se o menor valor $F$ parcial não for menor que $F_{\text {out }}$, o processo é interrompido e a equação em análise neste passo é a selecionada.

O método Eliminação Backward é mais econômico computacionalmente que o método Todas Possíveis Regressões. Além disso, proporciona aos analistas a possibilidade de observar o efeito de todas as variáveis preditoras na equação de regressão e o impacto da exclusão de cada variável. No entanto, uma desvantagem é que a utilização de todas as variáveis preditoras pode acarretar o aparecimento de multicolinearidade. 


\subsection{Stepwise}

O método Stepwise é uma melhora do método de Seleção Forward, pois após cada entrada de uma nova variável preditora, é verificada a significância de todas as variáveis que já estavam presentes na equação. A entrada de uma nova variável pode tornar outra variável, já presente na equação, não significativa. Assim, uma vez introduzida um nova variável, calcula-se os valores da estatística $F$ parcial para todas as variáveis preditoras da equação. O menor desses valores é comparado com $F_{\text {ovt }}$ e se for não significante, a correspondente variável é eliminada. Caso contrário, prossegue-se com o método Seleção Forward.

\section{Regras para entrada e saída de variáveis}

A escolha dos valores de corte $F_{I N}$ e $F_{\text {OUT }}$ nos métodos Seleção Forward, Eliminação Backward e Stepwise é um problema investigado por vários autores.

$\mathrm{O}$ valor de $\alpha$ de entrada dever ser selecionado de acordo com a necessidade do pesquisador da área e com muita cautela. Se selecionamos um $\alpha$ pequeno, $\alpha<0,05$, reduzimos a chance de variáveis preditoras pouco importantes entrarem na equação. Já se selecionamos um $\alpha$ de entrada grande, mais variáveis preditoras entram na equação.

Não é razoável fixar $\alpha_{O U T}<\alpha_{I N}$, pois algumas vezes isso poderia levar à saída de uma variável que acabou de entrar no modelo. Alguns analistas fixam $\alpha_{O U T}>\alpha_{I N}$ para garantir a proteção de variáveis já inseridas na equação, isto é, para que elas não saiam do modelo facilmente. Em particular Draper e Smith (1998) sugerem $\alpha=0,05$ ou $\alpha=0,10$ para ambos os testes de entrada e saída, se o pacote de regressão admitir essa opção, como por exemplo, o pacote SAS. Se preferir escolher 
valores diferentes, seria viável observar o efeito da escolha no subconjunto obtido e fazer várias execuções usando valores diferentes para $F_{I N}$ e $F_{O U T}$. Alguns pacotes já fornecem um procedimento muito popular de adotar os valores de $F_{I N}$ e $F_{\text {OUT }}$ iguais a 4, que corresponde aproximadamente ao quantil de ordem 0,95 da distribuição $F$.

O Stepwise é um dos melhores métodos de seleção de variáveis, pois verifica a contribuição de todas as variáveis a cada passo, evitando a utilização de variáveis não significantes na equação. Mesmo assim, este método não dispensa uma análise criteriosa da equação escolhida.

\subsection{Press}

O procedimento Press é baseado na soma de quadrados do resíduo preditivo e pode ser considerado uma mistura do método Todas as Possíveis Regressões e análise de resíduos.

A soma de quadrados do resíduo preditivo é obtida eliminando-se a i-ésima observação do conjunto de dados e estimando todas as possíveis regressões com as $n-1$ observações restantes. Depois devemos usar cada uma das equações ajustadas para prever $y_{i}$, através do valor $\hat{y}_{(i)}$ de cada equação. Em seguida, determina-se o resíduo $e_{(i)}=y_{i}-\hat{y}_{(i)}$ para todas as possíveis equações. Repete-se o processo para cada observação, $\mathrm{i}=1,2, \ldots, \mathrm{n}$, produzindo um conjunto de $\mathrm{n}$ resíduos $e_{(1)}, e_{(2)}, \ldots, e_{(n)}$. Então, para cada possível modelo, a estatística Press é definida como a soma de quadrados dos $\mathrm{n}$ resíduos,

$$
\operatorname{Pr} e s s=\sum_{i=1}^{n}\left(y_{i}-\hat{y}_{(i)}\right)^{2} .
$$


As equações com o menor Press são consideradas as "melhores" equações, pois têm menor resíduo preditivo, visto que, quando os resíduos $\left(y_{i}-\hat{y}_{(i)}\right)$ são pequenos, então o quadrado de cada resíduo e a soma deles também o será. A "melhor" equação será a que ao mesmo tempo tenha o menor número de variáveis preditoras e baixo valor do Press.

\subsection{Ridge Regression}

$\mathrm{Na}$ seleção de modelos, o método de Ridge Regression ou Regressão em Cristas é aplicado para selecionar variáveis preditoras quando seus valores são altamente colineares, ou seja, quando existe forte relação linear entre algumas variáveis preditoras.

Seja o modelo de regressão linear múltipla

$$
Y=Z \beta+\varepsilon
$$

com $n$, tamanho da amostra; $q$, número de parâmetros; $Z$, matriz de regressão $(n \times q), n>q ; \underline{\sim}$, vetor de respostas $(n \times 1) ; \beta$, vetor de parâmetros $(q \times 1) ; \varepsilon$, vetor de erros $(n \times 1)$, e a suposição $\underset{\mathcal{E} \sim}{\sim} N_{n}\left(0, \sigma^{2} I_{n}\right)$ indica que $\underset{\mathcal{E}}{\varepsilon}$ tem distribuição normal n-variada com vetor de médias 0 e matriz de covariância $\sigma^{2} I_{n}$.

Alguns autores sugerem que as variáveis preditoras sejam padronizadas, pois assim evita-se uma diferença grande entre os valores das estimativas dos coeficientes de regressão do modelo, e desta maneira pode-se comparar diretamente as variáveis quanto à sua significância. Após esta padronização, a matriz $Z^{\prime} Z$ fornecerá as correlações entre as variáveis preditoras. Consequientemente, todos os elementos da diagonal principal dessa matriz devem ser iguais a um. Além disso, se 
não há relação linear entre as variáveis preditoras, a matriz $Z$ é ortogonal, ou seja, $Z^{\prime} Z=I$. Porém, para o estudo de estimadores de Ridge, a matriz $Z$ na maioria dos casos é não ortogonal.

O método de estimação de Ridge (Oishi, 1983) consiste em somar uma constante $\lambda$, normalmente um valor no intervalo $[0,1]$, aos elementos da diagonal principal da matriz de correlação $Z^{\prime} Z$. Os coeficientes estimados pelo método Ridge são obtidos minimizando-se a quantidade

$$
S Q \operatorname{Re} s(\hat{\beta})=(\underset{\sim}{Y-Z} \hat{\beta})^{\prime}(\underset{\sim}{Y-Z} \hat{\beta})
$$

sujeito à restrição

$$
\hat{\beta}^{\prime} \hat{\beta}=\Re^{2}
$$

sendo que o valor de $\Re$ depende de $\lambda$. Na minimização, aplica-se o método de Lagrange, que no caso, consiste em derivar a função

$$
F=(\underline{Y}-Z \hat{\beta})^{\prime}(\underset{\sim}{Y-Z} \hat{\beta})+\lambda\left(\hat{\beta}^{\prime} \hat{\beta}-\Re^{2}\right)
$$

em relação à $\hat{\beta}$ e igualar a zero, sendo $\lambda$ o multiplicador de Lagrange. Deste modo, obtém-se a equação que conduz para a solução de Ridge

$$
\hat{\beta}(\lambda)=\left(Z^{\prime} Z+\lambda I\right)^{-1} Z^{\prime} \underline{\sim},
$$


sendo $\lambda$ uma constante maior que zero; $Z^{\prime} Y$ o vetor que fornece as correlações entre as variáveis preditoras e a dependente; $\hat{\beta}(\lambda)$ o vetor de estimadores viesados de $\beta$ obtido pelo método Ridge, contanto que exista $\left(Z^{\prime} Z+\lambda I\right)^{-1}$. Verifica-se que à medida que a constante $\lambda$ se aproxima de 1 , a variância total dos estimadores, $\operatorname{VarT}(\hat{\beta}(\lambda))=\sum_{k=1}^{q} \operatorname{Var}\left(\hat{\beta}_{k}(\lambda)\right)$, decresce, enquanto o quadrado do viés cresce.

Quando $\lambda=0$,

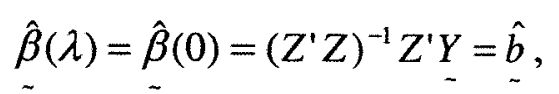

sendo $\hat{b}$ o vetor de estimadores de mínimos quadrados usual de $\underset{\sim}{\beta}$, com a condição de que $\left(Z^{\prime} Z\right)^{-1}$ exista.

Seja

$$
L^{2}(\lambda)=(\hat{\beta}(\lambda)-\beta)^{\prime}(\hat{\beta}(\lambda)-\beta,
$$

define-se o erro quadrático médio de $\hat{\beta}(\lambda)$ como

$$
E Q M(\hat{\beta}(\lambda))=E\left(L^{2}(\lambda)\right)=\operatorname{VarT}(\hat{\beta}(\lambda))+\operatorname{Viés}^{2}(\hat{\beta}(\lambda))
$$

ou seja, $\operatorname{EQM}(\hat{\beta}(\lambda))$ pode ser decomposto como a soma da variância com o quadrado do viés, $\operatorname{Viés}^{2}(\hat{\beta}(\lambda))=(E(\hat{\beta}(\lambda))-\beta)^{\prime}(E(\hat{\beta}(\lambda))-\beta)$. 
Se temos um estimador não viesado então o $E Q M$ desse estimador é idêntico à sua variância. Os estimadores de mínimos quadrados são não viesados, mas na presença de multicolinearidade são imprecisos, ou seja, altamente instáveis e com variância grande. Entretanto, os estimadores Ridge são muito mais precisos, mas têm um pequeno viés. De acordo com Neter, Kutner, Nachtsheim e Wasserman (1996), estimadores viesados com pequena variância são preferíveis a estimadores não viesados com grande variância, pois podem ter um menor erro quadrático médio.

De modo geral, em termos de $E Q M$, para os casos de alta multicolinearidade, $\hat{\beta}(\lambda)$ é melhor que $\underline{\hat{b}}$, pois $\operatorname{EQM}(\hat{\beta}(\lambda))<E Q M(\hat{b})$, para escolhas convenientes de $\lambda$. Por outro lado, para baixa multicolinearidade, $\hat{b}$ é melhor que $\hat{\beta}(\lambda)$.

Podemos notar também que, na presença de multicolinearidade, os valores dos estimadores de mínimos quadrados podem possuir magnitudes e sinais consideravelmente diferentes para diferentes amostras. Assim, tais estimadores são muito instáveis, e não são os mais adequados.

Para determinar $\lambda$, existem vários métodos, todos com vantagens $\mathrm{e}$ desvantagens. Se queremos estabilidade, devemos escolher a constante $\lambda$ através do método Traço da Crista, geralmente o mais usado e que foi proposto por Hoerl e Kennard (1970a). O Traço da Crista é o gráfico dos coeficientes estimados $\hat{\beta}(\lambda)$ contra os diferentes valores de $\lambda$.

Do ponto de vista de seleção de variáveis, através da utilização do traço, é possível selecionar um subconjunto de variáveis que produza estimativas mais estáveis que o conjunto completo. O procedimento consiste em eliminar aquelas variáveis que já não conseguem acrescentar mais informações, e só conseguem aumentar a instabilidade nas equações normais, devido à alta multicolinearidade presente. 
Hoerl e Kennard (1970b) introduziram algumas regras para eliminar as variáveis explicativas que supostamente causam instabilidade na equação:

a) Eliminar as variáveis que possuem coeficientes estáveis, mas com baixo poder de previsão, ou seja, variáveis com coeficientes de regressão padronizados pequenos;

b) Eliminar as variáveis que têm coeficientes instáveis e que sejam pouco importantes por apresentarem coeficientes próximo de zero, e

c) Eliminar uma ou mais variáveis com coeficientes mais instáveis, quando persistir alguma instabilidade ou o subconjunto resultante não for o desejado.

Enfim, devemos escolher o menor valor para $\lambda$ de modo a tornar as estimativas dos coeficientes de regressão estáveis no Traço da Crista, sem um aumento radical no erro quadrático médio e perda significativa em $R^{2}$ (coeficiente de explicação do modelo).

Devemos salientar que, em aplicações, o método de regressão Ridge não tem normalmente sido usado como um procedimento de seleção.

\subsection{Considerações finais}

Nas seções anteriores, procuramos destacar os métodos de seleção mais comuns na literatura, principalmente aqueles presentes nos livros de regressão linear. Devemos observar que os métodos de seleção não levam necessariamente à mesma solução.

Para modelos lineares generalizados, utiliza-se freqüentemente o método de Akaike (Akaike, 1974), que consiste em selecionar, dentre todos os modelos possíveis, aquele que minimiza a medida 


$$
A I C=-2 \ln L(\hat{\beta})+2 q
$$

sendo que $L(\hat{\beta})$ é a função de verossimilhança do modelo calculada no ponto $\beta=\hat{\beta}, \hat{\beta}$ estimador de máxima verossimilhança de $\beta$ e $q$ é o número de parâmetros do modelo.

No caso da regressão linear,

$$
A I C=n \ln \left(\frac{\sum_{i=1}^{n}\left(y_{i}-\hat{y}_{i}\right)^{2}}{n}\right)+2 q,
$$

$\operatorname{com} \sum_{i=1}^{n}\left(y_{i}-\hat{y}_{i}\right)^{2}$ sendo a soma de quadrados do resíduo do modelo. Destacamos a similaridade dessa medida com a quantidade $C_{q}$, apresentada na Seção 2.2 e por esse motivo, tal método não foi abordado no presente capítulo. 


\section{Capítulo 3}

\section{MÉTODOS DE SELEÇÃO DE VARIÁVEIS DO TIPO REDUÇÃO}

\subsection{Introdução}

Estimadores do tipo redução (Shrinkage estimators) são obtidos através de modificações nos estimadores usuais (máxima verossimilhança, não viciado de variância mínima, mínimos quadrados), com o objetivo de satisfazer a algum critério como minimizar o vício ou o erro quadrático médio.

De modo geral, tais estimadores são da forma

$$
\hat{\theta}_{r}=a\left[k \hat{\theta}+(1-k) \theta_{0}\right]
$$

com $0 \leq k \leq 1$, sendo que $\theta_{0}$ poderia ser, por exemplo, uma estimativa de $\theta$ baseada em experiências anteriores, para a qual deseja-se dar uma ponderação, $\hat{\theta}$ é o estimador usual de $\theta$ e $a$ é o fator de redução. 
$\mathrm{Na}$ literatura em Regressão, destacam-se o estimador ridge que, conforme discutiremos na Seção 3.3, pode ser escrito como $\hat{\theta}_{r}$, para $k=1$, $a=I-\lambda\left[Z^{\prime} Z+\lambda I\right]^{-1}$ e $\hat{\theta}=\left(Z^{\prime} Z\right)^{-1} Z^{\prime} Y$, e os estimadores do tipo James e Stein.

Neste capítulo, serão exibidos dois métodos de seleção de variáveis do tipo redução. O primeiro é o Garrote não Negativo, introduzido por Breiman (1995). O procedimento inicia com as estimativas de mínimos quadrados e as reduz por fatores cuja soma é restringida. Além disso, esses fatores, que denominaremos por $c_{1}, c_{2}, \ldots, c_{q}$, deverão ser não negativos. Para a realização do procedimento, foram utilizados os conceitos de erro de previsão, erro do modelo e seus estimadores. $\mathrm{O}$ segundo método foi proposto por Tibshirani (1996) e chamado de Lasso. Neste caso, escolhemos um valor para o parâmetro $s$, com o objetivo de controlar a quantidade de redução que é aplicada às estimativas dos $\beta_{k}, k=1,2, \ldots, q$. Para determinar o melhor valor para $s$, descreveremos futuramente os procedimentos CrossValidation e Cross-Validation Generalizado, que foram adotados pelo autor.

\subsection{Garrote não Negativo}

\subsubsection{Introdução e conceitos básicos}

A idéia usada no procedimento Garrote não Negativo por Breiman (1995) pode ser aplicada para conseguir redução nas estimativas dos $\beta_{k}$ ou seleção de variáveis preditoras. Na seleção de modelos através deste procedimento, são utilizados os conceitos de erro de previsão, erro do modelo e seus estimadores.

Para introduzir esses conceitos, vamos admitir que o modelo verdadeiro seja

$$
y_{i}=\mu\left(z_{i}\right)+\varepsilon_{i}
$$


$\operatorname{com} \varepsilon_{i}, i=1,2, \ldots, n$, variáveis aleatórias independentes e identicamente distribuídas com distribuição $N\left(0, \sigma^{2}\right) ; y_{i}$, variável resposta aleatória; $\mu\left(z_{i}\right)=\sum_{k=1}^{q} \beta_{k} z_{k i}$, equação de previsão verdadeira; $q$, número de variáveis preditoras; $\beta_{1}, \beta_{2}, \ldots, \beta_{q}$, parâmetros desconhecidos, e $\mathrm{z}_{\mathrm{i}}=\left(\mathrm{z}_{1 \mathrm{i}}, \mathrm{z}_{2 \mathrm{i}}, \ldots, \mathrm{z}_{\mathrm{qi}}\right)$ contém os valores das variáveis preditoras não aleatórias, para o i-ésimo elemento amostral, $i=1,2, \ldots, n$. Assumindo então os dados da forma $\left\{\left(\mathrm{y}_{\mathrm{i}}, \mathrm{z}_{\mathrm{i}}\right), \mathrm{i}=1,2, \ldots, \mathrm{n}\right\}$, sejam $\hat{\beta}_{k}, k=1,2, \ldots, q$, os estimadores de $\beta_{k}$, de modo que

$$
\hat{y}=\hat{\beta}_{1} z_{1}+\cdots+\hat{\beta}_{q} z_{q}
$$

Logo, a equação de previsão com base nas $n$ observações é da forma

$$
\hat{\mu}\left(z_{i}\right)=\sum_{k=1}^{q} \hat{\beta}_{k} z_{k i}
$$

sendo que $\hat{\beta}_{k}, \mathrm{k}=1,2, \ldots, \mathrm{q}$, são obtidos geralmente pelo método de mínimos quadrados usual.

Uma vez ajustado o modelo com base nos dados atuais, consideremos o experimento de produzir novos dados da forma

$$
y_{i}^{\text {NOVO }}=\mu\left(z_{i}\right)+\varepsilon_{i}^{\text {NOVO }} \text {, }
$$


com as mesmas variáveis preditoras e com variável resposta, $y_{i}^{\text {NOvO }}$, e erro, $\varepsilon_{i}^{\text {NOvO }}$, independente dos $\varepsilon_{i}, i=1,2, \ldots, n$, e com distribuição $N\left(0, \sigma^{2}\right)$ para $i=1,2, \ldots, n$. Admite-se adicionalmente que estas observações possuem os vetores de variáveis preditoras $z_{1}, z_{2}, \ldots, z_{n}$ presentes na amostra e são da forma $\left\{\left(\mathrm{y}_{\mathrm{i}}^{\text {NOvo }}, \mathrm{z}_{\mathrm{i}}\right), \mathrm{i}=1,2, \ldots, \mathrm{n}\right\}$.

O conceito do erro de previsão é definido como o erro de $\hat{\mu}\left(z_{i}\right)$ prevendo $y_{i}$, $i=1,2, \ldots, n$ a partir de $z_{i}$, para observações futuras não usadas na construção da equação de previsão. Se a equação de previsão, $\hat{\mu}\left(z_{i}\right)$, é gerada a partir das observações atuais, então o erro de previsão de um vetor $\hat{\mu}$ na previsão de $n$ novas observações, $y^{\text {Novo }}$, é definido como

$$
\begin{aligned}
& E P(\underline{\hat{\mu}})=E\left\|\underline{\sim}_{\sim}^{N O V O}-\hat{\mu}\right\|^{2}=E\left\{\sum_{i=1}^{n}\left[y_{i}^{N O V O}-\hat{\mu}\left(z_{i}\right)\right]^{2}\right\}, \\
& \text { com } \underline{y}^{\text {NOVO }}=\left[\begin{array}{c}
y_{1}^{\text {NOVO }} \\
y_{2}^{\text {NOVO }} \\
\vdots \\
y_{n}^{\text {NOvo }}
\end{array}\right] \text { e } \hat{\mu}=\left[\begin{array}{c}
\hat{\mu}\left(z_{1}\right) \\
\tilde{\mu}\left(z_{2}\right) \\
\vdots \\
\hat{\mu}\left(z_{n}\right)
\end{array}\right] \text {. Aplicando a equação (3.1), tem-se que } \\
& E P(\hat{\mu})=E\left\{\sum_{i=1}^{n}\left[\left(\mu\left(z_{i}\right)-\hat{\mu}\left(z_{i}\right)\right)^{2}+2 \varepsilon_{i}^{\text {Novo }}\left(\mu\left(z_{i}\right)-\hat{\mu}\left(z_{i}\right)\right)+\left(\varepsilon_{i}^{\text {Novo }}\right)^{2}\right]\right\}
\end{aligned}
$$

No cálculo dessa esperança, considera-se $\varepsilon_{i}^{\text {Novo }}$ e portanto $y_{i}^{\text {Novo }}$ como a única quantidade aleatória. Logo, a esperança é calculada sobre $y_{i}^{\text {Novo }}$, que é equivalente ao cálculo da esperança somente sobre $\varepsilon_{i}^{\text {Novo }}$. Vale lembrar que 
$E\left(\varepsilon_{i}^{\text {Novo }}\right)=0, \quad E\left(\left(\varepsilon_{i}^{\text {Novo }}\right)^{2}\right)=\sigma^{2}$ e $\hat{\mu}\left(z_{i}\right)$ funcionará como uma constante nessa esperança, desta maneira temos que

$$
E P(\hat{\mu})=n \sigma^{2}+\sum_{i=1}^{n}\left(\underset{\sim}{\mu\left(z_{i}\right)-\hat{\mu}\left(z_{i}\right)}\right)^{2}
$$

Devemos salientar que para o cálculo do erro de previsão de $y_{i}$, para $i=1,2, \ldots, n$ no modelo $y_{i}=\mu\left(z_{i}\right)+\varepsilon_{i}$, usa-se também a equação (3.2). Analisando-se esta expressão, observamos que o primeiro termo é o erro de previsão ligado à variância de $\varepsilon_{i}^{\text {Novo }}$ e o segundo é o erro de previsão devido à falta de ajuste com relação ao modelo verdadeiro. Pode-se definir o segundo termo como o erro do modelo, isto é,

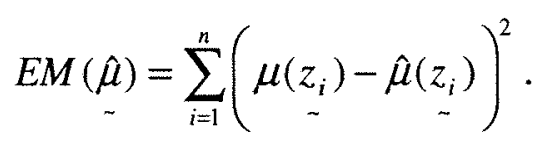

Escrevendo o erro do modelo na forma matricial, tem-se

$$
\begin{aligned}
E M(\hat{\mu}) & =\sum_{i=1}^{n}\left(\sum_{k=1}^{q} \beta_{k} z_{k i}-\sum_{k=1}^{q} \hat{\beta}_{k} z_{k i}\right)^{2} \\
& =\sum_{i=1}^{n}\left(\underline{\beta}^{\prime} \underline{\sim}_{i}-\hat{\beta}_{\sim} z_{i}\right)^{2},
\end{aligned}
$$

$\operatorname{com} \beta=\left[\begin{array}{c}\beta_{1} \\ \beta_{2} \\ \vdots \\ \beta_{q}\end{array}\right], \underset{\sim}{\hat{\beta}}=\left[\begin{array}{c}\hat{\beta}_{1} \\ \hat{\beta}_{2} \\ \vdots \\ \hat{\beta}_{q}\end{array}\right]$ e $z_{i}=\left[\begin{array}{c}z_{1 i} \\ z_{2 i} \\ \vdots \\ z_{q i}\end{array}\right]$ vetores coluna de dimensão $q$. Assim, 


$$
\begin{aligned}
& E M(\hat{\mu})=\sum_{i=1}^{n}\left((\underline{\beta-\hat{\beta}})^{\prime} z_{i}\right)^{2} \\
& =\sum_{i=1}^{n}\left((\underline{\beta-\hat{\beta}})^{\prime} z_{i} z_{i}^{\prime}(\underline{\beta}-\underline{\beta})\right) \\
& =(\underline{\beta}-\hat{\beta})^{\prime}\left[z_{1} z_{1}{ }^{\prime}+z_{2} z_{2}{ }^{\prime}+\cdots+z_{n} z_{\sim}^{\prime}{ }^{\prime}\right](\underline{\beta}-\hat{\beta}),
\end{aligned}
$$

e com isso, temos que

$$
E M(\hat{\mu})=(\underset{\sim}{\beta}-\hat{\beta})^{\prime} Z^{\prime} Z(\underset{\sim}{\beta}-\hat{\beta})
$$

pois $Z^{\prime}=\left[\begin{array}{cccc}z_{1} & z_{2} & \cdots & z_{n} \\ - & \sim & & -\end{array}\right], Z=\left[\begin{array}{c}z_{1}{ }^{\prime} \\ \tilde{z} \\ z_{2} \\ \vdots \\ z_{n} \\ \sim\end{array}\right], z_{i}{ }^{\prime}=\left[\begin{array}{llll}z_{1 i} & z_{2 i} & \cdots & z_{q i}\end{array}\right] \operatorname{com} i=1,2, \ldots, n$

Seja $\hat{\mu}_{k}$ o previsor do conjunto de $n$ observações futuras com base no modelo com $k$ variáveis preditoras, $k=1,2, \ldots, q$. Considera-se duas possibilidades de estimar $E P\left(\hat{\mu}_{k}\right)$, a primeira é usar os métodos do tipo $C_{p}$ (Mallows, 1973) e a segunda é através do procedimento Little Bootstrap (Breiman, 1992). No método $C_{p}$, calcula-se a estimativa de $E P\left(\hat{\mu}_{k}\right)$ por 


$$
\hat{E P}\left(\hat{\mu}_{k}\right)=S Q \operatorname{Re} s(k)+2 k \hat{\sigma}^{2},
$$

sendo que $\hat{\sigma}^{2}$ é a estimativa de $\sigma^{2}$ com base no modelo completo e $S Q \operatorname{Re} s(k)=S Q \operatorname{Re} s\left(\hat{\mu}_{\sim}\right)=\sum_{i=1}^{n}\left(y_{i}-\hat{\mu}_{k}\left(z_{i}\right)\right)^{2}$. Neste caso, $\hat{\mu}_{k}\left(z_{i}\right)$ é o estimador de $\mu_{k}\left(z_{i}\right)$ com base no procedimento mínimos quadrados usual ajustado ao modelo com $k$ variáveis preditoras e $S Q \operatorname{Re} s(k)$ a soma de quadrados do resíduo desse modelo.

Breiman (1992) mostra que $\hat{E P}\left(\hat{\mu}_{k}\right)$ é um estimador viesado para $E P\left(\hat{\mu}_{k}\right)$, e por essa razão não é eficiente na seleção de modelos. Segundo o autor, a melhor estimativa é obtida através da aplicação do método Little Bootstrap, que fornece estimativas com viés pequeno para o erro de previsão, qualquer que seja o procedimento de estimação de $\beta$ utilizado. A justificativa da expressão do estimador $\hat{E P}\left(\hat{\mu}_{k}\right)$ encontra-se em Breiman (1996a) e será apresentada a seguir.

Observa-se que

$$
\begin{aligned}
\operatorname{SQRe} s(\hat{\mu})= & \sum_{i=1}^{n}\left(y_{i}-\hat{\mu}\left(z_{i}\right)\right)^{2}=\sum_{i=1}^{n}\left(\mu\left(z_{i}\right)+\varepsilon_{i}-\hat{\mu}\left(z_{i}\right)\right)^{2} \\
& =\sum_{i=1}^{n} \varepsilon_{i}^{2}+2 \sum_{i=1}^{n}\left[\varepsilon_{i}\left(\mu\left(z_{i}\right)-\hat{\mu}\left(z_{i}\right)\right)\right]+\sum_{i=1}^{n}\left(\mu\left(z_{i}\right)-\hat{\mu}\left(z_{i}\right)\right)^{2} \\
& =\sum_{i=1}^{n} \varepsilon_{i}^{2}+2 \sum_{i=1}^{n} \varepsilon_{i} \mu\left(z_{i}\right)-2 \sum_{i=1}^{n} \varepsilon_{i} \hat{\mu}\left(z_{i}\right)+\sum_{i=1}^{n}\left(\mu\left(z_{i}\right)-\hat{\mu}\left(z_{i}\right)\right)^{2},
\end{aligned}
$$

conseqüentemente, 


$$
\sum_{i=1}^{n}\left(\underset{\sim}{\mu\left(z_{i}\right)-\hat{\mu}\left(z_{i}\right)}\right)^{2}=S Q \operatorname{Re} S(\hat{\mu})-\left[\sum_{i=1}^{n} \varepsilon_{i}^{2}+2 \sum_{i=1}^{n} \varepsilon_{i} \mu\left(z_{i}\right)-2 \sum_{i=1}^{n} \varepsilon_{i} \hat{\mu}\left(z_{i}\right)\right]
$$

e então

$$
\underset{\sim}{E} \underset{\hat{\mu}}{)}=n \sigma^{2}-\sum_{i=1}^{n} \varepsilon_{i}^{2}-2 \sum_{i=1}^{n} \varepsilon_{i} \mu\left(z_{i}\right)+S Q \operatorname{Re} s(\hat{\mu})+2 \sum_{i=1}^{n} \varepsilon_{i} \hat{\mu}\left(z_{i}\right)
$$

É importante lembrar que os elementos $\varepsilon_{i}$ e $S Q \operatorname{Re} s(\hat{\mu})$ presentes nesta expressão estão sendo considerados não aleatórios, de modo que $E P(\hat{\mu})$ é um parâmetro a ser estimado. Temos que $E\left[n \sigma^{2}-\sum_{i=1}^{n} \varepsilon_{i}^{2}-2 \sum_{i=1}^{n} \varepsilon_{i} \mu\left(z_{i}\right)\right]=0$, pois $E\left[\sum_{i=1}^{n} \varepsilon_{i}^{2}\right]=n \sigma^{2}$, e $\mu\left(z_{i}\right)$ é não aleatório, o que implica que $E\left[\sum_{i=1}^{n} \varepsilon_{i} \mu\left(z_{i}\right)\right]=0$, então o problema de estimar $E P(\hat{\mu})$ se reduz à obtenção do estimador

$$
\hat{E P}(\hat{\mu})=S Q \operatorname{Re} s(\hat{\mu})+2 \hat{E}\left[\sum_{i=1}^{n} \varepsilon_{i} \hat{\mu}\left(z_{i}\right)\right]
$$

$\operatorname{com} S Q \operatorname{Re} s(\hat{\mu})=\sum_{i=1}^{n}\left(y_{i}-\hat{\mu}\left(z_{i}\right)\right)^{2}$ e $\hat{E}\left[\sum_{i=1}^{n} \varepsilon_{i} \hat{\mu}\left(z_{i}\right)\right]$ um estimador de $E\left[\sum_{i=1}^{n} \varepsilon_{i} \hat{\mu}\left(z_{i}\right)\right]$.

Para aplicar o procedimento Little Bootstrap na obtenção de $\hat{E P}(\hat{\mu})$, adiciona-se a variável aleatória $\tilde{\mathcal{E}}_{i}$ a $y_{i}$, obtendo $\tilde{y}_{i}=y_{i}+\widetilde{\varepsilon}_{i}, \operatorname{com} \tilde{\mathcal{\varepsilon}}_{i}, i=1,2, \ldots, n$, variáveis aleatórias independentes e identicamente distribuídas com distribuição $N\left(0, t^{2} \hat{\sigma}^{2}\right)$; com $t$ constante, $\left.\left.t \in\right] 0,1\right] ; \hat{\sigma}^{2}$, estimativa da variância do erro obtida do 
modelo com todas as variáveis preditoras, e $\hat{\mu}\left(z_{i}\right)$, previsor de $y_{i}$ determinado com base na amostra original e no modelo em análise. A partir dos dados $\left(\tilde{\mathrm{y}}_{\mathrm{i}}, \mathrm{z}_{\mathrm{i}}\right)$, determina-se a equação de previsão $\widetilde{\mu}\left(z_{i}\right)$ através do método adotado, e calcula-se a quantidade $\frac{1}{t^{2}} \sum_{i=1}^{n} \widetilde{\varepsilon}_{i} \widetilde{\mu}\left(z_{i}\right)$. Repetindo-se esse procedimento várias vezes, a melhor estimativa para $E P(\hat{\mu})$ é

$$
\hat{E P}(\hat{\hat{\mu}})=S Q \operatorname{Re} s(\hat{\mu})+2 B_{t}
$$

sendo que $B_{\imath}$ é a média dos valores de $\frac{1}{t^{2}} \sum_{i=1}^{n} \widetilde{\varepsilon}_{i} \widetilde{\mu}\left(z_{i}\right)$ obtidos nas várias repetições.

Em particular, no caso do procedimento Ridge, obtém-se a equação de previsão $\hat{\mu}_{k^{*}}$ utilizando o método Ridge, que depende dos valores dos parâmetros $\lambda_{k^{*}}, k^{*}=1,2, \ldots, K \leq q$, sendo que $K$ é o número de possíveis valores para $\lambda$. A estimativa para o erro preditivo via little bootstrap é neste caso

$$
S Q \operatorname{Re} s\left(\lambda_{k^{*}}\right)+2 B_{t}\left(\lambda_{k} \cdot\right)
$$

sendo que $B_{t}\left(\lambda_{k}\right.$.) é calculado como descrito anteriormente e $S Q \operatorname{Re} s\left(\lambda_{k}\right.$.) é a soma de quadrados do resíduo do modelo ajustado sob o método Ridge com parâmetro $\lambda_{k}$.

Foi mostrado em Breiman (1992) que, para o método de Seleção de Subconjuntos, quanto menor $t$, menor o viés da estimativa de $E P\left(\hat{\mu}_{k^{*}}\right)$ via little 
bootstrap. Por outro lado, em regressão Ridge, a melhor estimativa não viesada para $E P\left(\hat{\mu}_{k^{*}}\right)$ é

$$
\hat{E P}\left(\hat{\mu}_{\underline{*}^{*}}\right)=S Q \operatorname{Re} s\left(\lambda_{k^{*}}\right)+2 \hat{\sigma}^{2} \operatorname{tr}\left(Z^{\prime} Z\left(Z^{\prime} Z+\lambda_{k^{*}} \cdot\right)^{-1}\right),
$$

a qual é utilizada para selecionar equações de regressão $\hat{\mu}_{\underline{-}^{*}} \operatorname{com} E P\left(\hat{\mu}_{k^{*}}\right)$ próximo do mínimo dos erros de previsão.

O número efetivo de parâmetros, ou seja, o número de variáveis preditoras, pode ser aproximado. Vale lembrar, que quando se trata dos estimadores de mínimos quadrados $\hat{\beta}=\left(Z^{\prime} Z\right)^{-1} Z^{\prime} Y$, então o previsor dos valores da variável dependente para os elementos amostrais é dado por $\hat{y}=Z\left(Z^{\prime} Z^{-1} Z^{\prime}{ }_{\sim}^{Y}\right.$, com $\operatorname{tr}\left(Z\left(Z^{\prime} Z\right)^{-1} Z^{\prime}\right)=q$, que é o número de variáveis preditoras no modelo ajustado. A mesma lógica foi usada nos estimadores Ridge, $\hat{\beta}=\left(Z^{\prime} Z+\lambda_{k} \cdot I^{-1} Z^{\prime} \underline{\sim}_{\sim}\right.$, e portanto os valores de $\lambda_{k}$. examinados por Breiman (1995) em suas simulações são tais que $\operatorname{tr}\left(Z\left(Z^{\prime} Z+\lambda_{k^{\prime}}\right)^{-1} Z^{\prime}\right)=\operatorname{tr}\left(Z^{\prime} Z\left(Z^{\prime} Z+\lambda_{k^{\prime}} \cdot\right)^{-1}\right)=1,2, \ldots, q$, sendo $Z$, matriz de regressão $(n \times q)$ e a última igualdade vale devido ao resultado $\operatorname{tr}(C B)=\operatorname{tr}(B C)$, quando $C B$ e $B C$ são produtos matriciais possíveis.

O comportamento de $B_{t}$ para $t$ pequeno é um reflexo da estabilidade do processo de regressão usado. Isso foi bastante explorado por Breiman (1996a).

\subsubsection{Seleção de modelos através do método Garrote não Negativo}


O método Garrote não Negativo consiste em obter inicialmente uma seqüência de funções de previsão, $\hat{\mu}_{1}(z), \ldots, \hat{\mu}_{K}(z)$, baseadas nos valores fixados dos $K$ parâmetros $s_{1}, \ldots, s_{K}$, respectivamente, e, ao mesmo tempo, nos estimadores de mínimos quadrados usuais, $\hat{\beta}_{k}^{0}, \mathrm{k}=1,2, \ldots, \mathrm{q}$. Para cada valor de $s$, que pode ser escolhido no conjunto $\{1,2, \ldots, q\}$, determina-se os coeficientes não negativos $c_{1}, c_{2}, \ldots, c_{q}$ de modo a minimizar a quantidade

$$
\sum_{i=1}^{n}\left(y_{i}-\sum_{k=1}^{q} c_{k} \hat{\beta}_{k}^{0} z_{k i}\right)^{2}
$$

sujeito às restrições

$$
\mathrm{c}_{\mathrm{k}} \geq 0 \text { para } \mathrm{k}=1,2, \ldots, \mathrm{q} \quad \text { e } \quad \sum_{k=1}^{q} c_{k} \leq s, \operatorname{com} 0<s<q .
$$

Breiman (1995) propõe uma sub-rotina em Fortran que tem como saída os valores dos $c_{1}, c_{2}, \ldots, c_{q}$ para qualquer valor de $\mathrm{s}, 0<s<q$. Quanto menor o valor de $s$, mais valores de $c_{k}$ são nulos e, como conseqüência, as variáveis preditoras associadas a esses valores são eliminadas, o que implicaria numa seleção de variáveis. Os valores de $c_{k}$ restantes, de modo geral, reduzem as estimativas dos coeficientes de regressão associados às variáveis preditoras. Além disso, tais coeficientes, da forma $c_{k} \hat{\beta}_{k}^{0}$, são na maioria dos casos menores que quando o método de mínimos quadrado usual é utilizado. O caso em que $s=q$ corresponderia à "mínima eliminação de variáveis preditoras".

Para avaliar a eficiência de seu método, inicialmente, o autor analisou a situação em que se conhece hipoteticamente o valor verdadeiro de $\operatorname{EP}\left(\hat{\mu}_{k^{*}}\right)$, 
$k^{*}=1,2, \ldots, K$, ou seja, em que $E P\left(\hat{\mu}_{1}\right), E P\left(\hat{\mu}_{2}\right), \ldots, E P\left(\hat{\mu}_{K}\right)$ são conhecidos. Tais modelos são por ele denominados de modelos "Bola de Cristal", por assumirem conhecido o valor verdadeiro de $E P\left(\hat{\mu}_{k^{*}}\right)$. Nesse caso, o modelo selecionado será aquele com menor valor de $E P\left(\hat{\mu}_{k^{*}}\right), k^{*}=1,2, \ldots, K$.

Vale ressaltar que na prática não conhecemos o valor verdadeiro do erro de previsão, portanto seu valor deve ser estimado. Uma possibilidade é usar o processo de Little Bootstrap no caso do Garrote não Negativo para se obter a estimativa para $E P\left(\hat{\mu}_{k^{*}}\right)$. Conforme comentado na seção anterior, a melhor estimativa para $E P\left(\hat{\mu}_{k^{*}}\right)$ é

$$
\hat{E P}\left(\hat{\mu}_{k^{*}}\right)=S Q \operatorname{Re} s\left(s_{k^{*}}\right)+2 B_{t}\left(s_{k^{*}}\right),
$$

sendo

$$
B_{t}\left(s_{k^{*}}\right)=\frac{1}{t^{2}} \hat{E}\left(\sum_{i=1}^{n} \tilde{\varepsilon}_{i} \tilde{\mu}_{k^{*}}\left(z_{i}\right)\right)
$$

obtido através da média de 25 repetições do método Little Bootstrap. Além disso, determina-se a soma de quadrados de resíduo de $s_{k^{*}}$ por $S Q \operatorname{Re} s\left(s_{k^{*}}\right)=S Q \operatorname{Re} s\left(\hat{\mu}_{k^{*}}\right)=\sum_{i=1}^{n}\left(y_{i}-\hat{\mu}_{k^{*}}\left(z_{-}\right)\right)^{2}, k^{*}=1,2, \ldots, K$ e $\hat{\mu}_{k^{*}}\left(z_{i}\right)$ é a equação de previsão obtida pelo método Garrote não Negativo com parâmetro $s_{k^{*}}$. Breiman (1992) sugere que $t$ pertença ao intervalo $[0,6 ; 0,8]$. 
No estudo de simulação, Breiman (1995) utilizou $s_{k^{*}}=1,2, \ldots, 20$, ou seja, $K=20$. Dessa forma, para se obter a estimativa de $E P\left(\hat{\mu}_{k^{*}}\right)$ e selecionar modelos através do procedimento Garrote não Negativo, são aplicados os seguintes passos:

1. Fixar $K$ valores para $s, s_{1}, s_{2}, \ldots, s_{K}$, com $0<s_{k^{*}} \leq q$ para $k^{*}=1,2, \ldots, K$;

2. Para cada valor de $s_{k^{*}}$, aplicar o método Garrote não Negativo determinando coeficientes não negativos $c_{1}, c_{2}, \ldots, c_{q}$ de modo a minimizar a quantidade descrita em (3.7);

3. Obter a seqüência de funções de previsão, $\hat{\mu}_{1}(z), \ldots, \hat{\mu}_{K}(z)$, com base nos modelos ajustados para cada um dos parâmetros $s_{1}, \ldots, s_{K}$;

4. Calcular $S Q \operatorname{Re} s\left(s_{1}\right), \ldots, S Q \operatorname{Re} s\left(s_{K}\right)$, ou seja, a soma de quadrados do resíduo para cada um dos $K$ modelos ajustados;

5. Gerar $\widetilde{\varepsilon}_{i}, \quad \mathrm{i}=1, \ldots, \mathrm{n}$, variáveis aleatórias independentes e identicamente distribuídas com distribuição $N\left(0, t^{2} \hat{\sigma}^{2}\right)$;

6. Formar a variável $\tilde{y}_{i}=y_{i}+\widetilde{\varepsilon}_{i}$ para as $n$ observações;

7. Usar os novos dados $\left(\tilde{\mathrm{y}}_{\mathrm{i}}, \mathrm{z}_{\mathrm{i}}\right)$ para encontrar as estimativas $\tilde{\mu}_{1}\left(z_{i}\right), \ldots, \tilde{\mu}_{K}\left(z_{i}\right)$ do método Garrote não Negativo que estão associadas aos valores dos parâmetros $\operatorname{dos} s_{1}, \ldots, s_{K}$;

8. Calcular a quantidade $\frac{1}{t^{2}} \sum_{i=1}^{n} \tilde{\varepsilon}_{i} \tilde{\mu}_{k^{*}}\left(z_{i}\right)$;

9. Para cada valor da sequiência de $s_{k^{*}}, k^{*}=1,2, \ldots, K$, repetir várias vezes os passos 5 a 8. De acordo com Breiman (1992) deve-se repetir o procedimento pelo menos 25 vezes e assim, para cada $s_{k}$. estaremos obtendo as médias dos 25 
valores da quantidade $\frac{1}{t^{2}} \sum_{i=1}^{n} \tilde{\varepsilon}_{i} \tilde{\mu}_{k^{*}}\left(z_{i}\right)$, que serão indicadas por $B_{t}\left(s_{1}\right)_{,}^{i}, B_{t}\left(s_{K}\right)$, respectivamente;

10. As estimativas via little bootstrap, $\hat{E P}\left(\hat{\mu}_{1}\right), \ldots, \hat{E P}\left(\hat{\mu}_{K}\right)$, são calculadas usando a equação (3.8), para cada um dos $K$ modelos obtidos pelo procedimento Garrote não Negativo;

11. Uma vez determinadas as estimativas de $\hat{E P}\left(\hat{\mu}_{\underline{k}^{*}}\right)$, a equação de regressão selecionada será aquela que dentre os modelos associados à seqüência $\hat{\mu}_{k^{*}}$, minimizar a estimativa de $E P\left(\hat{\mu}_{k^{*}}\right), k^{*}=1,2, \ldots, K$.

De acordo com Breiman (1992), em termos de seleção, o método Little Bootstrap não é muito eficiente quando há muitas variáveis preditoras pouco importantes no modelo, mas certamente é melhor que qualquer outro método. São consideradas variáveis pouco importantes aquelas que possuem coeficientes estimados próximos de zero e, principalmente, quando elas são correlacionadas com outras variáveis preditoras.

Uma interessante análise foi o estudo de simulação feito por Breiman (1995) usando 20 variáveis preditoras e 40 observações. Seu objetivo era comparar a eficiência dos métodos de Seleção de Subconjuntos (Melhor Subconjunto de Regressões/Forward/Backward/Stepwise), Garrote não Negativo e Regressão de Ridge.

Para comparar os métodos, foram fixados cinco diferentes conjuntos de coeficientes de regressão $\left(\beta_{1}, \beta_{2}, \ldots, \beta_{20}\right)$ e com base neles, foram gerados os dados $y_{i}=\sum_{k=1}^{q} \beta_{k} z_{k i}+\varepsilon_{i}$, sendo que $\varepsilon_{i}, \mathrm{i}=1, \ldots, \mathrm{n}$, são variáveis aleatórias independentes e identicamente distribuídas com distribuição $\mathrm{N}(0,1)$. Admitindo que na simulação os 
cinco conjuntos são indexados pela variável $h$, em cada conjunto, os coeficientęs $\beta_{1}, \beta_{2}, \ldots, \beta_{20}$ foram colocados em dois grupos, o primeiro, centrado na quinta variável, com valores dados por

$$
\beta_{5+j}=\left\{\begin{array}{cc}
(h-|j|)^{2}, & |j| \leq h \\
0, & h<|j| \leq 4
\end{array}\right.
$$

e o outro grupo, centrado na décima quinta variável, tal que

$$
\beta_{15+j}=\left\{\begin{array}{cc}
(h-|j|)^{2}, & |j| \leq h \\
0, & h<|j| \leq 5
\end{array}\right.
$$

para $h=1,2, \ldots, 5$.

Observa-se que, à medida que se aumenta o valor de $h(1,2, \ldots, 5)$, aumenta-se a quantidade de coeficientes diferentes de zero no conjunto. Cada grupo tem $2 h-1$ coeficientes diferentes de zero. Esta construção, para os cinco conjuntos $(1,2, \ldots, 5)$, forneceu respectivamente as quantidades de coeficientes diferentes de zero para $o$ grupo centrado na quinta variável, e a mesma quantidade para o grupo centrado na décima quinta variável: $1,3,5,7,9$.

Os valores de $Z$ foram gerados segundo uma distribuição $N(0, \Gamma)$, sendo que $\Gamma_{k k^{\prime}}=\rho^{|k-k|}$ são os elementos da matriz de covariância. Para cada valor de $h$, foram feitas 250 iterações, gerando 250 conjuntos de dados. Em cada uma das 250 iterações feitas, $\rho$ foi obtido através da distribuição uniforme no intervalo $[-1,1]$. Aqui, o objetivo de gerar a distribuição de $Z$ é apenas para a obtenção dos valores das variáveis preditoras, pois vale lembrar que nessa abordagem, $Z$ não é variável aleatória. 
O procedimento descrito anteriormente foi usado para selecionar modelos através de cada método a ser comparado: Seleção de Subconjuntos (Eliminação Backward), Garrote não Negativo e Regressão Ridge. Em cada uma das 250 iterações, a mesma quantidade de modelos foi obtida através de cada método, e em seguida as estimativas dos erros dos modelos, $\hat{E M}$, foram calculadas.

Da expressão (3.2) temos que $E P=n \sigma^{2}+E M$. Como consequiência, $\hat{E M}=\hat{E P}-n \hat{\sigma^{2}}$, com $\hat{\sigma}^{2}$ sendo o quadrado médio do resíduo do modelo e $\hat{E P}$ obtido através de (3.8). Os valores de $\hat{E P}$, nos dois primeiros métodos, foram estimados usando 25 repetições de Little Bootstrap com $t=0,6$. No caso do método de Ridge, os valores estimados do erro do modelo foram obtidos através da estimativa do erro de previsão fornecido pela equação (3.6). Em cada método, para cada modelo selecionado, foi calculado o valor verdadeiro do erro do modelo através da equação (3.3).

Gráficos para visualização dos resultados da simulação são apresentados a seguir. Nesta análise, o método mais adequado será o que apresentar a menor estimativa do erro do modelo, para todos valores de $h$ ou para a maior parte deles.

A Figura 3.1 exibe a média dos 250 valores do verdadeiro erro do modelo usando os subconjuntos selecionados pelas estimativas $E P$, em cada método e para cada valor de $h$. É possível ver nessa figura que o método Garrote não Negativo é sempre mais preciso que o Backward. Se há somente alguns coeficientes de regressão não nulos, caso em que $h<3$, então o procedimento Garrote não Negativo fornece boa previsão. Para muitos coeficientes não nulos, o método Ridge é o melhor. 
Figura 3.1 Média dos 250 valores dos erros dos modelos dos subconjuntos selecionados usando as estimativas do erro preditivo contra os valores de $h$ :

-, Backward; $\diamond$, Garrote não Negativo; +, Ridge.

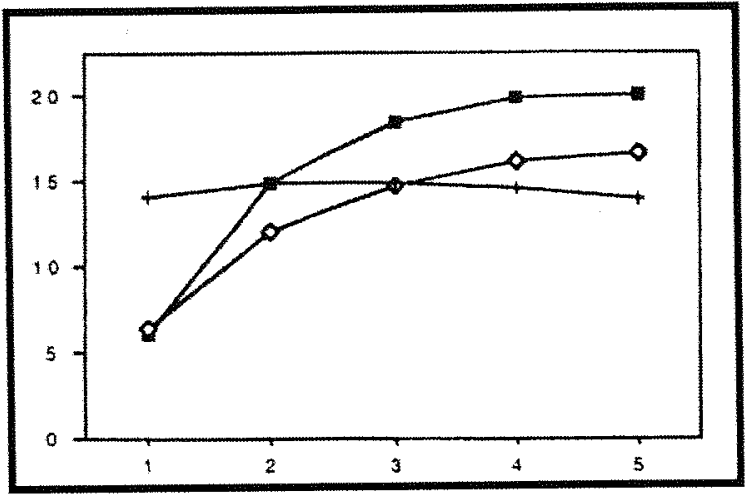

Numa etapa seguinte, foram obtidos os melhores subconjuntos de tamanho $1,2, \ldots, q$, ou seja, os subconjuntos com o mínimo valor da estimativa $E P$ com uma, duas, ..., $q$ variáveis preditoras, totalizando os $q$ melhores subconjuntos. Posteriormente, determinou-se o subconjunto que forneceu o mínimo do verdadeiro erro do modelo, obtido pela equação (3.3), entre os $q$ melhores subconjuntos, para os três métodos e para cada valor de $h$. Na Figura 3.2, encontra-se o gráfico dos verdadeiros erros dos modelos (procedimento "Bola de Cristal") dos subconjuntos selecionados em cada método e para cada $h$.

Comparando os erros exibidos na Figura $3.1 \mathrm{com}$ os da Figura 3.2, nota-se na segunda figura valores observados menores que os apresentados na primeira. $O$ autor observou que o método de Backward e o Garrote não Negativo na Figura 3.2 possuem o mínimo dos verdadeiros erros do modelo muito próximos. Além disso, o método de Ridge não se mostra eficiente comparado aos outros dois métodos, sendo melhor apenas quando temos muitos coeficientes não nulos, caso em que $h>3$. Por outro lado, o método de Ridge, usando as estimativas do erro de previsão como método de seleção, mostra-se mais eficiente nessa situação. 
Figura 3.2 Média dos 250 valores dos erros dos modelos dos subconjuntos selecionados usando seu verdadeiro valor "Bola de Cristal" contra os valores de $h$ :

-, Backward;, , Garrote não Negativo; +, Ridge.

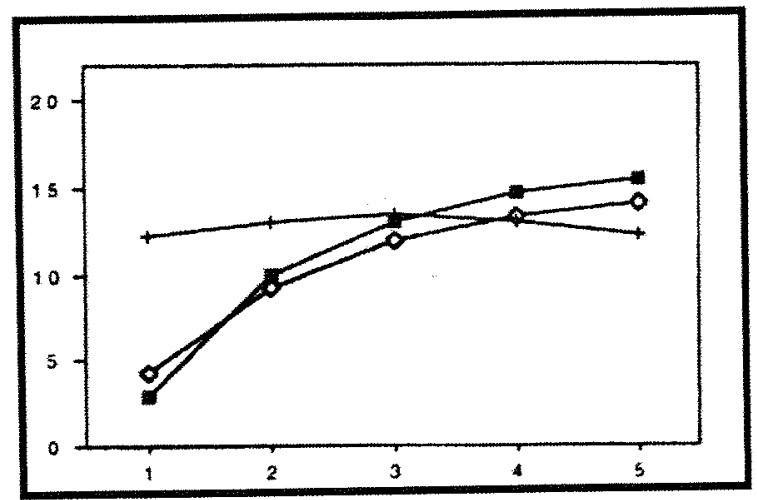

A Figura 3.3 mostra a diferença entre o erro do modelo obtido usando as estimativas EP em relação ao obtido usando o procedimento "Bola de Cristal".

Figura 3.3 Diferença entre as estimativas do erro preditivo e seu verdadeiro valor "Bola de Cristal" contra os valores de $h$ :

ш, Backward; $\diamond$, Garrote não Negativo; +, Ridge.

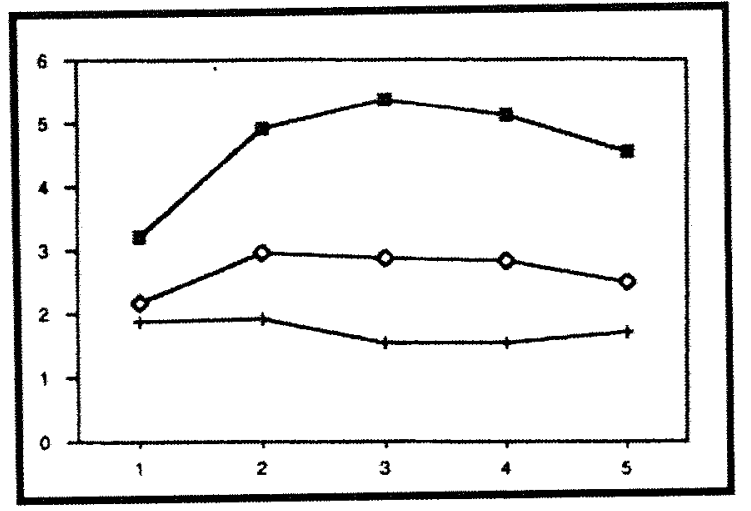

As estimativas do erro do modelo foram usadas tanto para selecionar modelos, quanto para fornecer informação sobre o erro desconhecido de cada um deles. Com o objetivo de verificar a eficiência dessas estimativas, são apresentados 
na Figura 3.4 a média dos valores absolutos das diferenças entre o erro do modelo estimado e o verdadeiro, em função de cada valor de $h$, para cada método. Dessa forma, calculou-se

$$
\frac{\sum_{I=1}^{250} \mid \hat{E M_{I}-E M_{I} \mid}}{250}
$$

em que $\hat{E M_{I}}$ e $E M_{I}$ são respectivamente as estimativas e o verdadeiro valor do erro para o modelo selecionado na $I$-ésima iteração. Nota-se que, para qualquer valor de $h$, as estimativas do erro do modelo no método de Backward são as piores. Por outro lado, o método de Ridge fornece a melhor estimação do erro do modelo, e o método Garrote não Negativo é o intermediário.

Figura 3.4 Erro médio absoluto na estimativa do erro do modelo para os subconjuntos selecionados contra os valores de $h$ :

-, Backward; $৩$, Garrote não Negativo; +, Ridge.

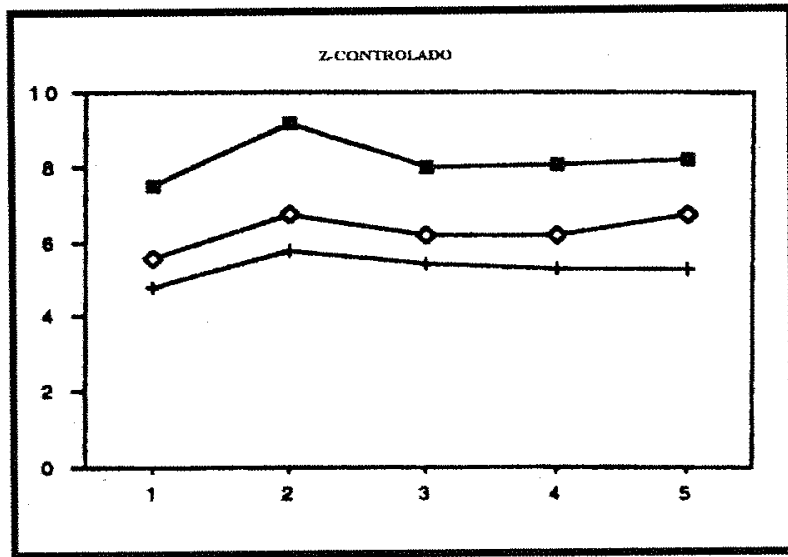

Finalizando, para comparar os métodos de Melhor Subconjunto de Regressão, segundo o algoritmo de menor soma de quadrados do resíduo de Furnival e Wilson 
(1974), e a Eliminação Backward, Breiman (1995) utilizou os dados simulados anteriormente. Em cada uma das 250 iterações, selecionou-se um subconjunto segundo o método Backward e a menor soma de quadrados de resíduo. Os erros desses modelos foram estimados usando Little Bootstrap (25 repetições, $t=0,6$ ).

Ao calcular as médias dos 250 valores dessas estimativas, observou-se que apesar do método Melhor Subconjunto de Regressão possuir soma de quadrados de resíduo baixo, a diferença com relação ao método Backward é pequena. Verificou-se que soma de quadrados de resíduo baixo, não significa que o erro de previsão também será baixo. Ou seja, se escolhemos as $M$ melhores regressões com base na minimização da soma de quadrados de resíduo, os valores dos erros dos modelos podem variar muito. Assim, é preciso ficar atento não apenas à $S Q \operatorname{Re} s$ mas também ao valor de $E M$.

Breiman (1995) mostrou depois de um extenso estudo com simulação, que o procedimento Garrote não Negativo tem na maioria das vezes o erro de previsão mais baixo que o método Backward. Além disso, o método Garrote não Negativo. possui erro de previsão menor que o método Ridge, exceto quando o modelo tem muitos coeficientes diferentes de zero, caso em que, por tender a anular coeficientes, pode não ser eficaz. Quando se usa o erro de previsão verdadeiro, ou seja, o procedimento "Bola de Cristal" na seleção de modelos, as diferenças em exatidão entre os três métodos são fortemente reduzidas, o que é natural, já que uma fonte de variabilidade está sendo eliminada.

O objetivo do procedimento Garrote não Negativo é reduzir os coeficientes a zero (e conseqüentemente, eliminar variáveis) ou simplesmente os diminuir. Se há somente alguns coeficientes de regressão não nulos, então o Garrote não Negativo fornece boa previsão. Entretanto, se há muitos coeficientes não nulos, o método Ridge é melhor que o Garrote não Negativo. Além disso, o procedimento Garrote 
não Negativo fornece mais coeficientes não nulos que o Backward, mas a perda em simplicidade é compensada pelo ganho essencial na precisão.

Finalizando, destacamos que a idéia do método Garrote não Negativo pode ser aplicada para conseguir outro método de redução, denominado por Breiman (1996a) de método Garrote. Os dois métodos Garrote não Negativo e Garrote são muitos similares, a diferença está nas restrições. No caso do novo método, o Garrote, os coeficientes $\mathrm{c}_{1}, \mathrm{c}_{2}, \ldots, \mathrm{c}_{\mathrm{q}}$ podem ser negativos e a restrição se torna $\sum_{k=1}^{q} c_{k}^{2} \leq s$. Espera-se que este método seja uniformemente mais exato que o método de Ridge e quase estável (vale ressaltar que Hoerl e Kennard (1970a) mostram que o método Ridge minimiza $\sum_{i=1}^{n}\left(y_{i}-\sum_{k} \beta_{k} z_{k i}\right)^{2}$ sob a restrição $\left.\sum_{k=1}^{q} \beta_{k}^{2} \leq s\right)$. O autor propõe estudar esta nova versão de Garrote em trabalhos futuros, porém até o momento, não há registro do estudo.

\subsection{Lasso}

O procedimento Lasso (least absolute shrinkage and selection operator) foi proposto por Tibshirani (1996) e surgiu a partir do método Garrote não Negativo. Desta forma, existem semelhanças entre os dois métodos, mas também importantes diferenças. Na descrição do procedimento, o autor admite que as variáveis preditoras são padronizadas de modo que $\sum_{i=1}^{n} \frac{z_{i k}}{n}=0$ e $\sum_{i=1}^{n} \frac{z_{i k}^{2}}{n}=1$.

No modelo

$$
y_{i}=\beta_{0}+\sum_{k=1}^{q} \beta_{k} z_{k i}+\varepsilon_{i}
$$


$\operatorname{com} \varepsilon_{i}, i=1,2, \ldots, n$, variáveis aleatórias independentes e identicamente distribuídas com distribuição $N\left(0, \sigma^{2}\right)$, as estimativas dos parâmetros $\beta_{0}, \beta_{1}, \ldots, \beta_{q}$ através do procedimento Lasso, $\hat{\beta}_{0}, \hat{\beta}_{1}, \ldots, \hat{\beta}_{q}$, são definidas a partir da minimização da quantidade

$$
\sum_{i=1}^{n}\left(y_{i}-\beta_{0}-\sum_{k=1}^{q} \beta_{k} z_{k i}\right)^{2}
$$

sujeita à condição

$$
\sum_{k=1}^{q}\left|\beta_{k}\right| \leq s, s \geq 0
$$

Devido à padronização, para todo $s$, temos que $\hat{\beta}_{0}=\bar{y}$. Sem perda de generalidade, podemos assumir que $\bar{y}=0$ e então omitir $\beta_{0}$.

$\mathrm{O}$ valor do parâmetro $s$ controla a quantidade de redução que é aplicada às estimativas dos $\beta_{k}, k=1,2, \ldots, q$. Se $s$ é muito grande, a restrição não tem efeito e, portanto, a solução será os estimadores de mínimos quadrados usuais na regressão da variável resposta sobre todas as variáveis preditoras. Por outro lado, se $s$ é muito baixo, então as estimativas de mínimos quadrados usuais serão reduzidas, e às vezes, alguns coeficientes de regressão, $\hat{\beta}_{k}$, podem ser nulos, implicando na eliminação da correspondente variável preditora $Z_{k}$.

Escolher um valor para o parâmetro $s$ é equivalente a selecionar o número de preditores a serem usados no modelo de regressão. Para determinar o melhor valor para $s$, descreveremos futuramente os procedimentos Cross-Validation e CrossValidation Generalizado, que foram adotados pelo autor. 
Na comparação do Lasso com o método Garrote não Negativo, o último tem uma desvantagem, pois sua solução depende ao mesmo tempo do sinal e da magnitude das estimativas de mínimos quadrados usuais. Sendo assim, em situações em que os estimadores de mínimos quadrados usuais não são eficientes, como por exemplo, no caso de existência de multicolinearidade, a qualidade de procedimento Garrote não Negativo ficará comprometida. Já, o método Lasso evita o uso explícito dessas estimativas, tornando-se um procedimento menos vulnerável ao comportamento das mesmas.

No caso ortonormal, em que $Z$ é a matriz de regressão $(n \times q)$ tal que $Z: Z=I$, sendo $I$ a matriz identidade, foram estudados os fatores de redução com relação ao método de mínimos quadrados usuais, nas estimativas dos coeficientes de regressão dos métodos Lasso, Seleção de Subconjuntos, regressão de Ridge, Garrote não Negativo e Garrote. Apresentamos a seguir a análise para cada método.

As estimativas dos parâmetros $\beta_{k}, \quad k=1,2, \ldots, q$, obtidas através do procedimento Lasso são dadas por

$$
\hat{\beta}_{k}=\left\{\begin{array}{ccc}
\operatorname{sinal}\left(\hat{\beta}_{k}^{0}\right)\left(\left|\hat{\beta}_{k}^{0}\right|-\lambda\right), & \text { se }\left|\hat{\beta}_{k}^{0}\right|-\lambda \geq 0 \\
0, & \text { caso } & \text { contrário }
\end{array}\right.
$$

sendo que $\hat{\beta}_{k}^{0}$ são as estimativas de mínimos quadrados dos parâmetros do modelo completo, e $\lambda$ é determinado pela restrição $\sum_{k=1}^{q}\left|\hat{\beta}_{k}\right|=s$. No Apêndice A, apresentamos a justificativa da expressão (3.9). A partir dela, observamos que se os coeficientes de mínimos quadrados estimados são, em valor absoluto, menores que $\lambda$, então as correspondentes estimativas dos parâmetros $\beta_{k}, k=1,2, \ldots, q$, via 
procedimento Lasso são iguais a zero. Os coeficientes restantes, que não são anulados, são reduzidos através do procedimento.

De acordo com Leblanc e Tibshirani (1998), a solução através do método Seleção de Subconjuntos é tal que o melhor subconjunto com $M$ variáveis, $1 \leq M \leq q$, contém as variáveis com os $M$ maiores valores absolutos das estimativas de mínimos quadrados dos coeficientes de regressão, $\left|\hat{\beta}_{k}^{0}\right|$, e coloca todos os demais coeficientes iguais a zero. Matematicamente, os coeficientes de regressão estimados podem ser escritos como

$$
\hat{\beta}_{k}=\hat{\beta}_{k}^{0} I\left\{\mid \hat{\beta}_{k}^{0} \geq \lambda\right\}, k=1,2, \ldots, q,
$$

para algum $\lambda \geq 0$, sendo que $I\{\}$ é a função indicadora.

Na regressão de Ridge, a solução é obtida através da minimização de

$$
\sum_{i=1}^{n}\left(y_{i}-\sum_{k=1}^{q} \beta_{k} z_{k i}\right)^{2}
$$

sujeita à restrição

$$
\sum_{k=1}^{q} \beta_{k}^{2} \leq s
$$

A relação entre os estimadores de Ridge, $\hat{\beta}$, e os de mínimos quadrados usuais, $\hat{\beta}^{0}$, está discutida em Hoerl e Kennard (1970a) e é dada pela expressão

$$
\hat{\beta}=\left\{I_{q}+\lambda\left(Z^{\prime} Z\right)^{-1}\right\} \hat{\beta}^{0}=\left\{I_{q}-\lambda\left[Z^{\prime} Z+\lambda I_{q}\right]^{-1}\right\} \hat{\beta}^{0} .
$$


Quando $Z^{\prime} Z=I_{q}$, então

$$
\begin{gathered}
\hat{\beta}=\left\{I_{q}-\lambda\left[I_{q}+\lambda I_{q}\right]^{-1}\right\} \hat{\beta}^{0}=\left\{I_{q}-\lambda\left[I_{q}(1+\lambda)\right]^{-1}\right\} \hat{\beta}^{0} \\
\hat{\beta}=\left\{I_{q}-\frac{\lambda}{1+\lambda} I_{q}\right\} \hat{\beta}_{\sim}^{0}=\left\{1-\frac{\lambda}{1+\lambda}\right\} \hat{\beta}^{0},
\end{gathered}
$$

levando às estimativas dos coeficientes de Ridge,

$$
\hat{\beta}_{k}=\frac{1}{1+\lambda} \hat{\beta}_{k}^{0}, k=1,2, \ldots, q
$$

sendo que $\lambda$ depende do valor $s$ através da restrição $\sum_{k=1}^{q} \hat{\beta}_{k}^{2} \leq s$. Pela expressão (3.10), o fator de redução é $\hat{\theta}\left(\hat{\beta}_{k}^{0}, \lambda\right)=\frac{1}{1+\lambda}$. Observa-se então que nesse caso, a regressão de Ridge produz um fator de redução constante para todo $k$.

No caso do método Garrote não Negativo, apresentado na expressão (3.7), quando $Z^{\prime} Z=I_{q}$, Breiman (1995) verifica que os valores dos coeficientes $c_{k}$ são dados por

$$
c_{k}=1-\frac{\lambda^{2}}{\hat{\beta}_{k}^{0^{2}}} \quad \text { e } \quad c_{k} \geq 0,
$$

sendo que $\lambda$ depende do valor $s$ através da condição $\sum_{k=1}^{q} c_{k}=s$. Dessa maneira, as estimativas para os coeficientes de regressão segundo este método seriam dadas por 


$$
\hat{\beta}_{k}=\left\{\begin{array}{ccc}
\left(1-\frac{\lambda^{2}}{\hat{\beta}_{k}^{0^{2}}}\right) \hat{\beta}_{k}^{0}, & \text { se } & 1-\frac{\lambda^{2}}{\hat{\beta}_{k}^{0^{2}}} \geq 0 \\
0, & \text { caso contrário. }
\end{array}\right.
$$

Pela expressão (3.11), o fator de redução dos coeficientes é $c_{k}=\hat{\theta}\left(\hat{\beta}_{k}^{0}, \lambda\right)=1-\frac{\lambda^{2}}{\hat{\beta}_{k}^{0^{2}}}$. Se $\mathrm{I} \hat{\beta}_{k}^{0} \leqslant \lambda$, então os correspondentes valores dos coeficientes de regressão, via método Garrote não Negativo, serão nulos, pois o fator de redução seria menor que zero. Na situação em que $\mid \hat{\beta}_{k}^{0} \triangleright \lambda$, quanto maior o valor de $\left|\hat{\beta}_{k}^{0}\right|$, maior o crescimento do fator de redução na direção do valor um. Neste caso, o fator de redução é uma função contínua em $\hat{\beta}^{0}$. Sempre que o fator de redução for igual a um, o coeficiente estimado pelo método em questão não sofrerá redução em relação ao coeficiente estimado pelo método de mínimos quadrados usuais.

De acordo com Breiman (1995), as estimativas dos coeficientes de regressão do método Garrote são dadas por

$$
\hat{\beta}_{k}=\frac{\hat{\beta}_{k}^{0^{2}}}{\hat{\beta}_{k}^{0^{2}}+\lambda^{2}} \hat{\beta}_{k}^{0}
$$

Assim, pela expressão (3.12), o fator de redução pode ser escrito como $\hat{\theta}\left(\hat{\beta}_{k}^{0}, \lambda\right)=\frac{\hat{\beta}_{k}^{0^{2}}}{\hat{\beta}_{k}^{0^{2}}+\lambda^{2}}$. 
Ao contrário do método Ridge, os procedimentos Garrote não Negativo e Garrote são de escala invariante, e espera-se que estes métodos sejam mais exatos que o método Ridge e quase estáveis. Um método de seleção de variáveis é dito escala invariante se o modelo selecionado quando as variáveis são $Y, Z_{1}, Z_{2}, \ldots, Z_{q}$ é o mesmo se forem utilizadas as variáveis transformadas $c Y, c Z_{1}, c Z_{2}, \ldots, c Z_{q}$, para qualquer constante $c \neq 0$. Métodos tradicionais como Stepwise, Forward e Backward são escala invariantes.

Tibshirani (1996) apresenta vários métodos para estimação do melhor valor para o parâmetro $s$ usado no método Lasso. No primeiro deles, utiliza o erro de previsão estimado através do procedimento Cross-Validation, que será discutido futuramente.

Nesse caso, para cada valor de $s$, calcula-se $s^{*}=\frac{s}{\sum_{k=1}^{q}\left|\hat{\beta}_{k}^{0}\right|}$ e a estimativa do erro de previsão para valores de $s^{*}$ no intervalo [0,1], pois $s<\sum_{k=1}^{q}\left|\hat{\beta}_{k}^{0}\right|$ para haver redução. O valor estimado para $s, \hat{s}^{*}$, será o do modelo que tiver o menor erro de previsão.

A segunda forma de estimar o parâmetro $s$ é a partir de uma aproximação linear para as estimativas de $\beta$ no procedimento Lasso. Com este intuito, a estimativa de $\beta$ sob esse método, $\hat{\beta}$, é aproximada por uma solução similar à obtida no método de regressão Ridge, escrevendo-se a restrição $\sum_{k=1}^{q}\left|\beta_{k}\right| \leq s$ como $\sum_{k=1}^{q} \frac{\beta_{k}^{2}}{\left|\beta_{k}\right|} \leq s$ e minimizando-se 


$$
F=(Y-Z \beta)^{\prime}(Y-Z \underline{\beta})+\lambda\left[\sum_{k=1}^{q} \frac{\beta_{k}^{2}}{\left|\beta_{k}\right|}-s\right]
$$

Nessas condições, se $\beta^{\prime}=\left[\begin{array}{llll}\beta_{0} & \beta_{1} & \cdots & \beta_{q}\end{array}\right]$, então segue que

$$
\sum_{k=1}^{q} \frac{\beta_{k}^{2}}{\left|\beta_{k}\right|}=\beta\left[\begin{array}{cccc}
\left|\beta_{0}\right| & 0 & 0 & 0 \\
0 & \left|\beta_{1}\right| & 0 & 0 \\
0 & 0 & \ddots & 0 \\
0 & 0 & 0 & \left|\beta_{q}\right|
\end{array}\right]^{-} \beta=\beta^{\prime} W^{-} \beta
$$

sendo que $W^{-}$é a inversa generalizada de $W=\operatorname{diag}\left(\left|\beta_{k}\right|\right)$. Na prática, os elementos de $W$ seriam substituídos por estimativas iniciais de $\beta_{0}, \beta_{1}, \ldots, \beta_{q}$.

Desta forma,

$$
F=Y^{\prime} Y-Y_{\sim}^{\prime} Z \beta-\beta^{\prime} Z^{\prime} Y+\beta^{\prime} Z^{\prime} Z \beta+\lambda \beta^{\prime} W^{-} \beta-\lambda s
$$

e como $Y^{\prime} Z \beta$ e $\beta^{\prime} Z^{\prime} Y$ são unidimensionais, então seus valores são iguais, e conseqüentemente, a função $F$ pode ser escrita como

$$
F=Y^{\prime} Y-2 Y^{\prime} Z \beta+\beta^{\prime} Z^{\prime} Z \beta+\lambda \beta^{\prime} W^{-} \beta-\lambda s
$$

Portanto,

$$
\frac{\partial F}{\partial \beta}=-2 Y^{\prime} Z+2 Z^{\prime} Z \beta+2 \lambda W^{-} \beta=0,
$$


e com isso,

$$
\hat{\beta}=\left(Z^{\prime} Z+\lambda \hat{W}^{-}\right)^{-1} Z^{\prime} \underset{\sim}{Y}
$$

com $\lambda$ dependendo de $s$ através da restrição $\sum_{k=1}^{q}\left|\hat{\beta}_{k}\right| \leq s$ e $\hat{W}^{-}$é a inversa generalizada de $\hat{W}=\operatorname{diag}\left(\left|\hat{\beta}_{k}\right|\right)$, sendo o estimador de $W^{-}$.

A matriz de covariância dos estimadores de $\beta$ pode então ser estimada por

$$
\left(Z^{\prime} Z+\lambda \hat{W}^{-}\right)^{-1} Z^{\prime} Z\left(Z^{\prime} Z+\lambda \hat{W}^{-}\right)^{-1} \hat{\sigma}^{2}
$$

sendo que $\hat{\sigma}^{2}$ é uma estimativa da variância do erro.

Conforme comentado anteriormente na Seção 3.2.1, se $\hat{\beta}=\left(Z^{\prime} Z+\lambda \hat{W}^{-}\right)^{-1} Z^{\prime} Y$, o número de variáveis preditoras no modelo ajustado pode ser aproximado por

$$
p(s)=\operatorname{traço}\left\{Z\left(Z^{\prime} Z+\lambda \hat{W}^{-}\right)^{-1} Z^{\prime}\right\} \text {, para cada } s \geq 0 \text { e com } \sum_{k=1}^{q}\left|\hat{\beta}_{k}\right| \leq s .
$$

A partir daí, calcula-se o valor da estatística do tipo Cross-Validation Generalizado, dada por

$$
C V G(s)=\frac{S Q \operatorname{Re} s(s)}{n(1-p(s) / n)^{2}},
$$


com $S Q \operatorname{Re} s(s)$ sendo a soma de quadrados do resíduo do modelo ajustado através do método Lasso com parâmetro $s$. O valor de $s$ mais adequado é escolhido de forma a minimizar $C V G(s)$.

Tibshirani (1996) apresentou um estudo de simulação em que compara os procedimentos de Mínimos Quadrados, Lasso, Garrote não Negativo, Melhor Subconjunto de Regressão e Regressão de Ridge. Nos procedimentos que envolviam a estimação de parâmetros de redução $(\lambda$ e $s)$, foi utilizado o método CrossValidation.

Inicialmente, os melhores subconjuntos de cada tamanho, $S_{0}, S_{1}, \ldots, S_{q}$, foram encontrados com base nos dados originais segundo os métodos: Mínimos Quadrados, Lasso (Cross-Validation), Lasso (Cross-Validation Generalizado), Garrote não Negativo, Seleção de Subconjuntos e Regressão de Ridge. Admitiu-se que os valores ajustados para o único modelo de $S_{0}$ são nulos, pois neste modelo, $\hat{y}=\bar{y}=0$.

O método Cross-Validation descrito por Zhang (1993) consiste em utilizar uma amostra de tamanho $n$ com $n=r d$, sendo que $r$ e $d$ são inteiros. Com este objetivo, a amostra é partida em $r$ subgrupos, $g_{1}, g_{2}, \ldots, g_{r}$ que são mutualmente exclusivos. Cada subgrupo possui $d$ observações.

Desta forma, usando o procedimento Cross-Validation com $r=5$ cinco partições foram definidos: $T$, conjunto com todos dados; $T^{v}$, conjunto teste com base na $v$-ésima partição, e $T-T^{v}$, conjunto de Cross-Validation, $v=1,2, \ldots, 5$. Desta maneira, a $v$-ésima partição era a que possuía $g_{v}$ como conjunto teste e a união dos demais como conjunto de Cross-Validation.

Para cada partição, foi selecionado o modelo através do método melhor subconjunto de cada tamanho $(0,1, \ldots, q)$, com base nos dados de $T-T^{v}$. Tais modelos foram denotados respectivamente por $S_{0}^{\nu}, S_{1}^{\nu}, \ldots, S_{q}^{\nu}$ e o procedimento foi 
repetido para $v=1,2, \ldots, 5$. Se $E P^{v}(p)$ é o erro de previsão quando $S_{p}^{v}$ é aplicado para prever os dados de $T^{v}$, obtém-se a estimativa para o erro de previsão, dada por

$$
\hat{E P}(p)=\frac{1}{5} \sum_{v=1}^{5} \hat{E P}^{v}(p), \text { para } p=0,1,2, \ldots, q
$$

O valor de $p$ que minimizar $\hat{E P}(p)$ será o valor estimado de $p, \hat{p}$, e o modelo selecionado será $S_{\hat{p}}$. Os valores de $\hat{E P}^{v}(p)$ serão obtidos como

$$
\sum_{y \in T^{v}}\left(y-\hat{y}_{T-T^{v}}\right)^{2},
$$

em que $\hat{y}_{T-T^{v}}$ é a previsão do valor de $y \in T^{v}$ obtida com base em $S_{p}^{v}$.

Foram analisados três casos no procedimento de simulação. No primeiro deles, o estudo foi aplicado em cinqüenta conjuntos de dados simulados com vinte observações cada e oito variáveis preditoras, de acordo com o modelo

$$
y_{i}=\sum_{k=1}^{q} \beta_{k} z_{k i}+\sigma \varepsilon_{i}
$$

em que $\varepsilon_{i}, i=1,2, \ldots, n$, tem distribuição normal padrão e as correlações entre $z_{k} \mathrm{e}$ $z_{k^{\prime}}, k, k^{\prime}=1, \ldots, q$ e $k \neq k^{\prime}$, foram $\rho^{|k-k|}$ com $\rho=0,5$. Tanto no primeiro caso quanto no segundo, foi assumido que $\sigma=3$. Já no terceiro caso, o desvio padrão foi menor que o dos dois primeiros, utilizou-se $\sigma=2$. Além disso, os valores para os vetores de coeficientes de regressão para os casos são, respectivamente, $\beta=(3 ; 1,5 ; 0 ; 0 ; 2$; $0 ; 0 ; 0) \beta_{k}=0,85$, para todo $k$, e $\beta=(5 ; 0 ; 0 ; 0 ; 0 ; 0 ; 0 ; 0)$. 
Os valores exibidos nas Tabelas 3.1, 3.2 e 3.3 foram obtidos através de 200 simulações do modelo e aplicação de cada método a cada conjunto de dados obtido. Na primeira coluna encontram-se os métodos analisados, na segunda, as medianas dos erros quadráticos médios de $\hat{\beta}$ para os 200 modelos ajustados de acordo com cada método. Determinado $\hat{\beta}$, vetor de estimativas de $\beta$ segundo cada método, o erro quadrático médio de $\hat{\beta}$ foi calculado como

$$
\operatorname{EQM}(\hat{\beta})=(\hat{\beta}-\beta)^{\prime}\left(Z^{\prime} Z\right)(\hat{\beta}-\beta)
$$

uma vez que $\beta$ é conhecido via simulação.

Ainda na Tabela 3.1, os números médios de coeficientes nulos estão na terceira coluna e na quarta, os valores médios de $\hat{s}^{*}$. Entre parênteses encontram-se os desvios padrões associados às respectivas quantidades calculadas nas 200 repetições.

Tabela 3.1: Resultados do caso 1

\begin{tabular}{lccc}
\hline \multicolumn{1}{c}{ Método } & $\begin{array}{c}\text { Mediana dos } \\
\text { erros quadráticos } \\
\text { médios }\end{array}$ & $\begin{array}{c}\mathrm{n}^{\circ} \text { médio de } \\
\text { coeficientes } \\
\text { nulos }\end{array}$ & $\hat{s}^{*}$ médio \\
\hline Mínimos Quadrados & $2,79(0,12)$ & 0,0 & - \\
Lasso (Cross-Validation) & $2,43(0,14)$ & 3,3 & $0,63(0,01)$ \\
Lasso & $\mathbf{1 , 9 3}(0,09)$ & 2,4 & $0,73(0,01)$ \\
(Cross-Validation Generalizado) & & & \\
Garrote não Negativo & $2,29(0,16)$ & 3,9 & - \\
Seleção de Subconjuntos & $2,44(0,16)$ & 4,8 & - \\
Regressão de Ridge & $3,21(0,12)$ & 0,0 & - \\
\hline
\end{tabular}


Os melhores métodos de regressão são aqueles com menor mediana dos erros quadráticos médios. Sendo assim, pode-se observar que, no primeiro caso, Tabela 3.1, os métodos Lasso (Cross-Validation Generalizado) e o Garrote não Negativo são os melhores, seguido pelos métodos Lasso (Cross-Validation) e Seleção de Subconjuntos.

Tabela 3.2: Resultados do caso 2

\begin{tabular}{lccc}
\hline \multicolumn{1}{c}{ Método } & $\begin{array}{c}\text { Mediana dos } \\
\text { erros quadráticos } \\
\text { médios }\end{array}$ & $\begin{array}{c}\mathrm{n}^{\circ} \text { médio de } \\
\text { coeficientes } \\
\text { nulos }\end{array}$ & $\hat{s}^{*}$ médio \\
\hline Mínimos Quadrados & $6,50(0,64)$ & 0,0 & - \\
Lasso (Cross-Validation) & $5,30(0,45)$ & 3,0 & $0,50(0,03)$ \\
Lasso & $4,87(0,35)$ & 2,3 & $0,69(0,23)$ \\
(Cross-Validation Generalizado) & & & \\
Garrote não Negativo & $7,40(0,48)$ & 4,3 & - \\
Seleção de Subconjuntos & $9,05(0,78)$ & 5,2 & - \\
Regressão de Ridge & $\mathbf{2 , 3 0}(0,22)$ & 0,0 & - \\
\hline
\end{tabular}

No segundo caso, Tabela 3.2, verifica-se que a regressão de Ridge é melhor que os outros métodos, apresentando a mediana dos erros quadráticos médios bem inferior em relação aos demais. 
Tabela 3.3: Resultados do caso 3

\begin{tabular}{lccc}
\hline \multicolumn{1}{c}{ Método } & $\begin{array}{c}\text { Mediana dos } \\
\text { erros quadráticos } \\
\text { médios }\end{array}$ & $\begin{array}{c}\mathrm{n}^{\circ} \text { médio de } \\
\text { coeficientes } \\
\text { nulos }\end{array}$ & $\hat{\mathbf{s}}^{*}$ médio \\
\hline Mínimos Quadrados & $2,89(0,04)$ & 0,0 & - \\
Lasso (Cross-Validation) & $0,89(0,01)$ & 3,0 & $0,50(0,03)$ \\
Lasso & $1,02(0,02)$ & 3,9 & $0,63(0,04)$ \\
(Cross-Validation Generalizado) & & & \\
Garrote não Negativo & $\mathbf{0 , 5 2}(0,01)$ & 5,5 & - \\
Seleção de Subconjuntos & $0,64(0,02)$ & 6,3 & - \\
Regressão de Ridge & $3,53(0,05)$ & 0,0 & - \\
\hline
\end{tabular}

Na Tabela 3.3, estão os resultados do terceiro caso, onde é possível observar que os métodos Garrote não Negativo e Seleção de Subconjuntos são os melhores, seguidos pelo método Lasso (Cross-Validation). Observa-se também que o método de Regressão Ridge é o pior, pois o valor da mediana dos erros quadráticos médios é ainda maior que o valor obtido pelo método de Mínimos Quadrados.

Desta forma, nos casos analisados, o autor trabalhou com três diferentes situações:

1. Trata-se do terceiro caso em que se tem poucas variáveis preditoras com coeficientes altos;

2. Refere-se ao primeiro caso, aqui se tem um número pequeno a moderado de variáveis preditoras no ajuste com coeficientes moderados, e

3. Segundo caso, no qual tem-se grande quantidade de variáveis preditoras com coeficientes pequenos. 
Com base na análise destes casos, verifica-se então que o método Lasso é um grande competidor dos procedimentos Seleção de Subconjuntos e da Regressão Ridge. Segundo Tibshirani (1996), a Seleção de Subconjuntos, como o método Lasso e o Garrote não Negativo, são mais vantajosos, pois produzem modelos mais interpretáveis que os obtidos pelo método Ridge.

No entanto, no método Ridge, temos um motivo para efetuar a redução, que é a presença de multicolinearidade. Já, o método Lasso restringe fortemente os estimadores de mínimos quadrados, que são ótimos, válidas certas condições. Desta forma, seria útil como método de seleção, quando se deseja trabalhar com poucas variáveis no modelo.

\subsection{Considerações finais}

Ainda na linha de métodos do tipo redução, Breiman (1996b) apresenta o procedimento Stacked Regression, que consiste em utilizar uma combinação linear das variáveis preditoras como uma nova e única variável. Neste caso, o problema de seleção de variáveis preditoras fica substituído pelo da determinação dos coeficientes da combinação linear.

Logo, a idéia do método Stacked é usar os procedimentos de CrossValidation e mínimos quadrados sob restrições para determinar os coeficientes na combinação. A finalidade é formar combinações lineares de diferentes preditores para melhorar a precisão de previsão. Se temos $q$ preditores, $v_{1}(z), v_{2}(z), \ldots, v_{q}(z)$, então ao invés de selecionar o melhor preditor desse conjunto, combina-se todos os preditores, usando um conjunto de teste ou usando o Cross-Validation para estimar o erro de previsão de cada $v_{q}(z)$ sobre os dados "futuros".

Verifica-se que este método é útil quando se ajusta o modelo com o objetivo de previsão, pois o previsor resultante tem erro quadrático médio baixo. Por não ser 
um método de seleção propriamente dito e por ter um enfoque distinto do adotaḍo nesse trabalho, tal procedimento não será aqui discutido. 


\section{Capítulo 4}

\section{MÉTODOS DE SELEÇÃO DE VARIÁVEIS COM ENFOQUE BAYESIANO}

\subsection{Introdução}

Apresentaremos neste capítulo alguns métodos "Bayesianos" de seleção de variáveis. O primeiro deles utiliza uma abordagem bayesiana para calcular $P\left(\beta_{k}=0\right)$, que representa a probabilidade do $k$-ésimo coeficiente de regressão ser nulo, caso em que a variável preditora $Z_{k}$ é eliminada do modelo.

O segundo é baseado na obtenção das densidades preditivas do vetor de dados não observados ou futuros. O critério conduz à escolha do modelo que possui o maior valor da medida de verossimilhança preditiva $L_{m}$ associada ao $m$-ésimo modelo, $m=1,2, \ldots, 2^{q}-1$.

No terceiro método, utiliza-se o modelo linear Bayesiano hierárquico completo, definido como uma mistura de normais. O objetivo neste caso é identificar indiretamente, a partir da distribuição a posteriori de um conjunto de 
variáveis indicadoras, usadas para apontar os modelos mais promissores, os subconjuntos de variáveis preditoras possíveis para o modelo. Os preditores com alta probabilidade a posteriori têm mais chance de entrar no modelo e estes poderão ser identificados pela alta frequiência na amostragem de Gibbs.

\subsection{Método Bayesiano}

O método que iremos descrever a seguir utiliza uma particular abordagem Bayesiana na seleção de variáveis preditoras no modelo de regressão linear. $O$ procedimento, apresentado com detalhes em Mitchell e Beauchamp (1988), consiste em assumir primeiramente uma distribuição de probabilidades para a variável dependente, a partir da especificação de uma família de distribuições a priori para os parâmetros desconhecidos $\left(\beta_{k}, k=1,2, \ldots, q\right.$, e $\left.\sigma^{2}\right)$, no modelo de regressão

$$
\mathrm{y}=\sum_{k=1}^{q} \beta_{k} z_{k}+\varepsilon
$$

Com base nas $q$ variáveis preditoras, obtém-se $2^{q}$ possíveis submodelos, sendo que cada submodelo exclui um conjunto particular de variáveis preditoras e inclui o restante. Seja $Z_{m}$ a matriz de regressão $\left(n \times q_{m}\right)$, contendo os valores dos preditores incluídos no $m$ - ésimo submodelo $A_{m}, m=1,2, \ldots, 2^{q}$. Com esta notação, ficam definidos os estimadores de mínimos quadrados do vetor de parâmetros correspondente, $\beta_{m}$, dados por

$$
\hat{\beta}_{m}=\left(Z_{m}{ }^{\prime} Z_{m}\right)^{-1} Z_{m}{ }^{\prime} \underline{-}
$$


e a soma de quadrados de resíduo

$$
S_{m}^{2}=\left(\underline{y}-Z_{m} \hat{\beta}_{m}\right)^{\prime}\left(y-Z_{m} \hat{\beta}_{m}\right)
$$

Admite-se que a variável aleatória $\varepsilon$ tem distribuição normal com média 0 e variância $\sigma^{2}$, e que $\varepsilon_{1}, \varepsilon_{2}, \ldots, \varepsilon_{n}$ são independentes. Dado $\beta$ e $\sigma^{2}, y$ tem distribuição normal multivariada com média $Z_{m} \beta_{m}$ e matriz de covariância $\sigma^{2} I$.

Cada submodelo é definido por uma parte dos $\beta_{1}, \beta_{2}, \ldots, \beta_{q}$ sendo nulos e os restantes, não nulos. Na seleção de variáveis são determinadas, através do Teorema de Bayes, probabilidades a posteriori para $\beta_{k}, k=1,2, \ldots, q$, e este cálculo permite encontrar um modelo ou alguns submodelos mais adequados. Além disso, obtém-se outras probabilidades interessantes para selecionar variáveis, como por exemplo, a probabilidade a posteriori de cada modelo, uma vez observados os dados.

Assume-se que o erro aleatório $\varepsilon$ tem distribuição normal com média zero e desvio padrão $\sigma$, sendo que $\ln (\sigma)$ é uniformemente distribuído no intervalo $\left[-\ln \left(\sigma_{0}\right), \ln \left(\sigma_{0}\right)\right]$, para $\sigma_{0}$ muito grande. Desta forma, atribuí-se a $\sigma$ uma distribuição a priori não informativa uniforme, que é usada quando não se dispõe de muita informação preliminar.

Admite-se ainda que os coeficientes de regressão do modelo são variáveis aleatórias mutuamente independentes com distribuição a priori independente de $\sigma$. Para cada coeficiente de regressão $\beta_{k}$ sujeito a ser eliminado do modelo, a distribuição a priori é uma mistura de um conjunto de coeficientes nulos e um outro conjunto com distribuição uniforme contínua em intervalos que não contêm o zero. Em resumo, supõe-se que $\beta_{k}$ é uniformemente distribuído entre os limites, $-f_{k}$ e $f_{k}$, exceto se $z_{k}$ é vulnerável à eliminação, pois neste caso, existiria uma 
probabilidade $h_{0 k}$ de tal parâmetro assumir valor zero. Os autores admitem valores de $f_{k}$ muito grandes, para aumentar a incerteza sobre o valor de $\beta_{k}$ naqueles modelos em que $z_{k}$ está presente.

Formalmente, para cada coeficiente de regressão $\beta_{k}$, a distribuição a priori é tal que

$$
\begin{gathered}
\operatorname{Pr}\left(\beta_{k}=0\right)=h_{0 k}, \\
\operatorname{Pr}\left(\beta_{k}<b, \beta_{k} \neq 0\right)=\left(b+f_{k}\right) h_{1 k}, \quad-f_{k}<b<f_{k}
\end{gathered}
$$

$\mathrm{e}$

$$
\operatorname{Pr}\left(\left|\beta_{k}\right|>f_{k}\right)=0
$$

com $h_{0 k}>0, h_{1 k}>0, h_{0 k}+2 h_{1 k} f_{k}=1$. Os valores $f_{k}$ e $\gamma_{k}=\frac{h_{0 k}}{h_{1 k}}$ são parâmetros da distribuição, valendo ainda as seguintes relações

$$
h_{1 k}=\left(\gamma_{k}+2 f_{k}\right)^{-1}
$$

$\mathrm{e}$

$$
\gamma_{k}=\frac{2 h_{0 k} f_{k}}{1-h_{0 k}}
$$

A partir daí, segue que a distribuição a priori de cada submodelo $A_{m}$ é dada por

$$
\begin{aligned}
\operatorname{Pr}\left(A_{m}\right)=\prod_{\bar{K}_{m}} h_{0 k} \prod_{K_{m}}\left(1-h_{0 k}\right) \\
=\prod_{\bar{K}_{m}} h_{0 k} \prod_{K_{m}}\left(2 f_{k} h_{1 k}\right),
\end{aligned}
$$


sendo que $K_{m}$ e $\bar{K}_{m}$ representam os conjuntos dos coeficientes de regressão respectivamente incluídos e excluídos do submodelo $A_{m}$.

Com a intenção de se obter a probabilidade a posteriori de $A_{m}$ dado $y$, foram calculadas algumas funções de verossimilhança. Através da função densidade de $y$, dado $A_{m}, \beta_{m}$ e $\sigma$, obteremos a função densidade de $y$, dado $A_{m}$. Esta última função, juntamente com a distribuição a priori de $A_{m}$, será usada para se determinar a probabilidade a posteriori de cada particular modelo $A_{m}$ dado $y$.

Descreveremos a seguir cada passo para obter a distribuição a posteriori de $A_{m}$ dado $y$.

$\underline{1^{\circ} \text { passo: }}$ Cálculo da função densidade de $y$, dado $A_{m}, \beta_{m}$ e $\sigma$

Seja $\hat{\beta}_{m}$ o estimador de mínimos quadrados de $\beta_{m}$ no modelo $A_{m}$. Nessas condições,

$$
\begin{aligned}
& \left(y-Z_{m} \beta_{m}\right)^{\prime}\left(y-Z_{m} \beta_{m}\right)= \\
& =\left[\left(y-Z_{m} \hat{\beta}_{m}\right)+\left(Z_{m} \hat{\beta}_{m}-Z_{m} \beta_{m}\right)\right]^{\prime}\left[\left(y-Z_{m} \hat{\beta}_{m}\right)+\left(Z_{m} \hat{\beta}_{m}-Z_{m} \beta_{m}\right)\right] \\
& =\left(\underset{\sim}{y} Z_{m} \hat{\beta}_{m}\right)^{\prime}\left(y-Z_{m} \hat{\beta}_{m}\right)+\left(y-Z_{m} \hat{\beta}_{m}\right)^{\prime}\left(Z_{m} \hat{\beta}_{m}-Z_{m} \beta_{m}\right)+ \\
& +\left(Z_{m} \hat{\beta}_{m}-Z_{m} \beta_{m}\right)^{\prime}\left(y-Z_{m} \hat{\beta}_{m}\right)+\left(Z_{m} \hat{\beta}_{m}-Z_{m} \beta_{m}\right)^{\prime}\left(Z_{m} \hat{\beta}_{m}-Z_{m} \beta_{m}\right)
\end{aligned}
$$

sendo que 


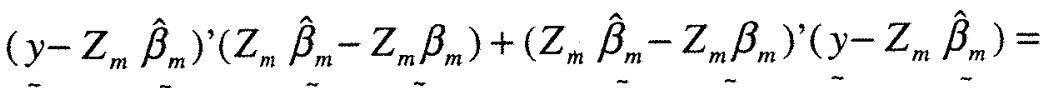

$$
\begin{aligned}
& =y^{\prime} Z_{m} \hat{\beta}_{m}-y^{\prime} Z_{m} \beta_{m}-\hat{\beta}_{m}{ }^{\prime} Z_{m}{ }^{\prime} Z_{m} \hat{\beta}_{m}+\hat{\beta}_{m}{ }^{\prime} Z_{m}{ }^{\prime} Z_{m} \beta_{m}+ \\
& +\hat{\beta}_{m}^{\prime} Z_{m}^{\prime} y-\hat{\beta}_{m}{ }^{\prime} Z_{m}^{\prime} Z_{m} \hat{\beta}_{m}-\beta_{m}^{\prime} Z_{m}^{\prime} y+\beta_{m}{ }^{\prime} Z_{m}{ }^{\prime} Z_{m} \hat{\beta}_{m} \\
& =y^{\prime} Z_{m} \hat{\beta}_{m}-y_{\sim}^{\prime} Z_{m} \beta_{m}-\hat{\beta}_{m}^{\prime} Z_{m}^{\prime} y+y_{\sim}^{\prime} Z_{m} \beta_{m}+ \\
& +\hat{\beta}_{m}{ }^{\prime} Z_{m}^{\prime} y-\hat{\beta}_{m}{ }^{\prime} Z_{m}{ }^{\prime} y-\beta_{\sim}{ }^{\prime} Z_{m}{ }^{\prime} y+\beta_{m}{ }^{\prime} Z_{m}{ }^{\prime} y \\
& =0 \text {. }
\end{aligned}
$$

pois $\left(Z_{m}{ }^{\prime} Z_{m}\right) \hat{\beta}_{m}=Z_{m}{ }^{\prime} y$ e $\hat{\beta}_{m}{ }^{\prime} Z_{m}{ }^{\prime} Z_{m}=y^{\prime} Z_{m}\left(Z_{m}{ }^{\prime} Z_{m}\right)^{-1}\left(Z_{m}{ }^{\prime} Z_{m}\right)=y^{\prime} Z_{m}$.

Desta forma, temos que

$$
\begin{aligned}
& \left(y-Z_{m} \beta_{m}\right)^{\prime}\left(y-Z_{m} \beta_{m}\right)= \\
& =\left(\underline{y}-Z_{m} \hat{\beta}_{m}\right)^{\prime}\left(y-Z_{m} \hat{\beta}_{m}\right)+\left(\beta_{\sim}-\hat{\beta}_{m}\right)^{\prime} Z_{m}{ }^{\prime} Z_{m}\left(\beta_{m}-\hat{\beta}_{m}\right) \\
& =S_{m}^{2}+\left(\beta_{m}-\hat{\beta}_{m}\right)^{\prime} Z_{m}^{\prime} Z_{m}\left(\beta_{m}-\hat{\beta}_{m}\right) \text {. }
\end{aligned}
$$

Logo,

$$
\begin{aligned}
f\left(y / A_{m}, \beta_{m}, \sigma\right)= & (2 \pi)^{-n / 2} \sigma^{-n} \exp \left[-\frac{1}{2 \sigma^{2}}\left(y-Z_{m} \beta_{m}\right)^{\prime}\left(y-Z_{m} \beta_{m}\right)\right] \\
& =(2 \pi)^{-n / 2} \sigma^{-n} \exp \left[-\frac{1}{2 \sigma^{2}}\left(S_{m}^{2}+\left(\beta_{m}-\hat{\beta}_{m}\right)^{\prime} Z_{m}^{\prime} Z_{m}\left(\beta_{m}-\hat{\beta}_{m}\right)\right)\right]
\end{aligned}
$$


20 passo: Seja $f\left(\beta_{m} / A_{m}, \sigma\right)$ a função densidade de $y$ dado $A_{m}$ e $\sigma$. Esta função é dada por

$$
f\left(\underline{y} / A_{m}, \sigma\right)=\int_{-\infty}^{\infty} f\left(y / A_{m}, \beta_{\sim}, \sigma\right) f\left(\beta_{\sim} / A_{m}, \sigma\right) d \beta_{\sim} .
$$

Como $f\left(\beta_{m} / A_{m}, \sigma\right)=\prod_{K_{m}}\left(2 f_{k}\right)^{-1}$, assumindo-se que cada $f_{m}$ é suficientemente grande a fim de que, para $\sigma<\sigma_{0}$, todas as integrais de $-f_{k}$ a $f_{k}$ podem ser substituídas por integrais de $-\infty$ a com erro pequeno, obtém-se

$$
f\left(\underset{\sim}{y} / A_{m}, \sigma\right)=\left(\prod_{K_{m}}\left(2 f_{k}\right)^{-1}\right)(2 \pi)^{-\left(n-q_{m}\right) / 2}\left|Z_{m}{ }^{\prime} Z_{m}\right|^{-1 / 2} \sigma^{-\left(n-q_{m}\right)} \exp \left[\frac{-S_{m}^{2}}{\left(2 \sigma^{2}\right)}\right],
$$

em que $q_{m}$ é o número de termos no submodelo $A_{m}$.

3० passo: Obtém-se a função densidade de $y$, dado o modelo $A_{m}$, multiplicando-se a densidade obtida no $2^{\circ}$ passo por $f\left(\sigma / A_{m}\right)$, que é proporcional a $\sigma^{-1}$, e integrandose com relação a $\sigma$. Após cálculos, verifica-se que

$$
\begin{aligned}
f\left(\underset{\sim}{y / A_{m}}\right) & =\int_{0}^{\infty} f\left(\underline{-}\left(A_{m}, \sigma\right) f\left(\sigma / A_{m}\right) d \sigma\right. \\
& =\left(2 \ln \left(\sigma_{0}\right)\right)^{-1}\left(\prod_{K_{m}}\left(2 f_{k}\right)^{-1}\right) \pi^{-\left(n-q_{m}\right) / 2}\left(\frac{1}{2}\right) \Gamma\left(\frac{n-q_{m}}{2}\right) \cdot\left|Z_{m} Z_{m}\right|^{-1 / 2}\left(S_{m}^{2}\right)^{-\left[\left(n-q_{m}\right) / 2\right]} .
\end{aligned}
$$


Na derivação de $f\left(y / A_{m}\right)$, assumiu-se que $\sigma_{0}$ é suficientemente grande a fïm de que o intervalo da integral sobre $\sigma$, que era de $\sigma_{0}^{-1}$ a $\sigma_{0}$, possa ser substituído por 0 a $\infty$ com erro pequeno.

4 passo: Nessa etapa, multiplica-se a densidade $f\left(y / A_{m}\right)$ por $\operatorname{Pr}\left(A_{m}\right)$ fornecida na equação (4.2), obtendo-se a densidade conjunta $f\left(y, A_{m}\right)$. A partir daí, determina-se a probabilidade a posteriori de $A_{m}$ dado $y$, dada por

$$
\begin{aligned}
\operatorname{Pr}\left(A_{m} / y\right) & =\frac{f\left(y, A_{m}\right)}{p(y)} \\
& =g\left(\prod_{\bar{K}_{m}} \gamma_{k}\right) \pi^{q_{m} / 2} \Gamma\left(\frac{n-q_{m}}{2}\right) \cdot\left|Z_{m}{ }^{\prime} Z_{m}\right|^{-1 / 2}\left(S_{m}^{2}\right)^{-\left[\left(n-q_{m}\right) / 2\right]},
\end{aligned}
$$

sendo que $p(y)$ é a densidade de $y$ e $g$ é uma constante normalizadora. Para o modelo sem variáveis preditoras $(\hat{y}=\bar{y})$, substitui-se o determinante presente na expressão (4.3) por 1 .

Supõe-se que os coeficientes de regressão correspondentes aos preditores vulneráveis têm distribuições a priori idênticas. A distribuição a posteriori depende somente dos dados e do parâmetro $\gamma$. Em relação à variável preditora implícita, o parâmetro $\gamma$ é igual a $h_{0}$ dividido por $h_{1}$ para a distribuição a priori comum, sendo que $h_{0}>0, h_{1}>0$. Como consequiência, $\gamma$ tem o mesmo valor para todos $\gamma_{k}$ 's positivos. Consideramos que $\gamma$ é um parâmetro ajustável do modelo de Bayes. Neste caso, não se atribuí a este parâmetro uma distribuição de probabilidade e nem se pretende escolher uma priori. 
Padronizando-se os preditores vulneráveis, então para todos submodelos tendo probabilidade a priori positiva, a probabilidade a posteriori fornecida na equação (4.3), torna-se

$$
\begin{aligned}
\operatorname{Pr}\left(A_{m} / \underset{\sim}{y}\right) & =g\left(\gamma^{q-q_{m}}\right) \pi^{q_{m} / 2} \Gamma\left(\frac{n-q_{m}}{2}\right) \cdot\left|Z_{m}{ }^{\prime} Z_{m}\right|^{-1 / 2}\left(S_{m}^{2}\right)^{-\left[\left(n-q_{m}\right) / 2\right]} \\
& =g^{\prime} \gamma^{-q_{m}} \pi^{q_{m} / 2} \Gamma\left(\frac{n-q_{m}}{2}\right) \cdot\left|Z_{m}{ }^{\prime} Z_{m}\right|^{-1 / 2}\left(S_{m}^{2}\right)^{-\left[\left(n-q_{m}\right) / 2\right]}
\end{aligned}
$$

sendo que $g$ ' é outra constante normalizadora.

Métodos gráficos foram propostos como guias informais para escolher $\gamma$, avaliando a importância das variáveis preditoras individualmente, o número de termos esperado no modelo, e o grau de incerteza sobre o submodelo mais adequado. Com este objetivo, Mitchell e Beauchamp (1988) construíram os gráficos das seguintes quantidades em função de $\gamma$ :
a) A probabilidade a posteriori de que um particular coeficiente de regressão seja nulo;
b) O número esperado de termos (a posteriori) no submodelo;
c) A perturbação (a posteriori) da distribuição do submodelo;
d) O erro preditivo a posteriori, e
e) A probabilidade a posteriori da qualidade do ajuste.

O item (a) avalia a importância de um determinado coeficiente de regressão, $\beta_{k}$, e utiliza a probabilidade a posteriori dada por

$$
\operatorname{Pr}\left(\beta_{k}=0 / y\right)=\sum \operatorname{Pr}\left(A_{m} / y\right)
$$


onde a soma é calculada sobre todos submodelos $A_{m}$ que não incluem o coeficiente $\beta_{k}$. Quando, para um particular valor de $\gamma$, a probabilidade a posteriori de um determinado coeficiente ser nulo atinge o valor 1 , este coeficiente de regressão é omitido do modelo.

A probabilidade a posteriori, dada na equação (4.4), pode ser usada para calcular o número esperado de termos (a posteriori) no submodelo, determinado por

$$
E\left(q_{m} / \underset{\sim}{y}\right)=\sum_{m=1}^{2 q} q_{m} \operatorname{Pr}\left(A_{m} / \underline{y}\right)
$$

que corresponde ao item (b). Valores baixos para $E\left(q_{m} / y\right)$ implicam num modelo mais simples.

A perturbação a posteriori da distribuição do submodelo no item (c) é definida como

$$
H=-\sum_{m=1}^{24} \operatorname{Pr}\left(A_{m} / y\right) \ln \left(\operatorname{Pr}\left(A_{m} / y\right)\right),
$$

e mede o grau de dispersão das probabilidades a posteriori entre os submodelos. Esta quantidade é usada para quantificar a incerteza sobre a escolha de um submodelo mais adequado. Foi usado na análise o antilogaritmo de $H$ por ser mais fácil de interpretar. Observe que se a probabilidade a posteriori for distribuída igualmente entre os $2^{q}$ submodelos, $\mathrm{O}$ antilogaritmo de $H$ será igual a $2^{q}$.

Grandes valores de $H$ indicam a existência de vários modelos com probabilidade a posteriori alta, o que proporciona uma confusão na escolha do modelo. Quanto menor for o valor do antilogaritmo de $H$, menor é o valor de $H$, e 
como consequiência, teremos poucos modelos com alta probabilidade a posteriori, diminuindo assim a incerteza da escolha.

A aplicação do método Bayesiano gerou uma classe de distribuições a posteriori (4.4), indexadas pelo parâmetro $\gamma$. Se os parâmetros $h_{0 k}$ e $h_{1 k}$ da distribuição de probabilidades de $\beta_{k}$ fossem conhecidos, $\gamma_{k}$ ficaria completamente determinado. Como isso geralmente não é possível, e para evitar a utilização de uma priori não informativa para $\gamma$, os autores sugeriram duas aproximações para se escolher o valor deste parâmetro. Tais aproximações foram utilizadas nos itens (d) e (e).

No item (d), tem-se o erro preditivo, que é determinado usando uma aproximação do tipo Cross Validation Bayesiano. O procedimento consiste em gerar para cada valor de $\gamma$ uma densidade preditiva $f_{(i)}\left(y_{i}\right)$ para a variável dependente $Y_{i}$, $i=1,2, \ldots, n$, com base em todos os dados exceto a própria observação. A qualidade da densidade preditiva pode ser avaliada comparando o valor previsto $\hat{y}_{i}$, com o verdadeiro valor observado da variável, $Y_{i}$, usando alguma função de perda $L\left(\hat{y}_{i}, Y_{i}\right)$, que tem uma distribuição gerada pela densidade preditiva $f_{(i)}$. Para perda quadrática, a média da distribuição de $L\left(\hat{y}_{i}, Y_{i}\right)$ é

$$
E Q M_{i}=E\left(\hat{y}_{i}-Y_{i}\right)^{2}
$$

sendo que a esperança é calculada com base em $f_{(i)}$.

Os cálculos básicos necessários para a avaliação de (4.5) encontram-se em Mitchell e Beauchamp (1988). Verifica-se que

$$
E Q M_{i}=\sum_{m=1}^{2^{q}} \operatorname{Pr}_{(i)}\left(A_{m}\right)\left[f_{i, m}^{2}+\left(S_{m}^{2}-e_{i, m} f_{i, m}\right)\left(1-h_{i, m}\right)^{-1}\left(n-q_{m}-3\right)^{-1}\right]
$$


com $f_{i, m}=e_{i, m} /\left(1-h_{i, m}\right)$, sendo que $e_{i, m}=y_{i, m}-\hat{y}_{i, m}$ é o $i$-ésimo elemento do vetor de resíduos de mínimos quadrados, $h_{i, m}$ é o $i$-ésimo elemento da diagonal da matriz $Z_{i, m}\left(Z_{i, m}^{\prime} Z_{i, m}\right)^{-1} Z_{i, m}^{\prime}$, e $\operatorname{Pr}_{(i)}\left(A_{m}\right)$ é a probabilidade a posteriori do modelo $m$ excluindo os dados da $i$-ésima observação.

Observa-se então que, todos os modelos com $\operatorname{Pr}\left(A_{m}\right)$ positiva contribuem no cálculo de $E Q M_{i}$. A posterior seleção do modelo é que, conforme veremos a seguir, dependerá dessa quantidade.

Para cada $Y_{i}$ no conjunto de dados, é calculado (4.5). Determina-se então como medida da eficiência do previsor $\hat{y}_{i}$, o erro preditivo, dado por

$$
E P=\sqrt{\frac{1}{n} \sum_{i=1}^{n} E Q M_{i}}
$$

O gráfico (d) proporciona uma avaliação visual do efeito do parâmetro $\gamma$ na capacidade preditiva da distribuição a posteriori e deve ser utilizado na determinação de $\gamma$. Altos valores do erro preditivo estariam associados a escolhas de $\gamma$ que dão alta probabilidade a posteriori para modelos com baixa capacidade preditiva. Dessa forma, deseja-se valores de $\gamma$ que fornecem erros preditivos baixos.

Analisando-se a expressão de $E Q M_{i}$, observa-se que o erro preditivo é finito somente se $n-q_{m}>3$. Além disso, a aproximação usada é satisfatória apenas quando há no mínimo quatro graus de liberdade para o erro.

Já no item (e), avalia-se a qualidade do ajuste através da medida $Q$, para cada possível valor de $\gamma$. Esta medida é a soma das probabilidades a posteriori de todos os submodelos que satisfazem o teste $F$ padrão para qualidade de ajuste com relação ao modelo completo, a um nível de significância especificado. O valor do 
parâmetro $\gamma$ é escolhido de forma a maximizar a qualidade de ajuste, ou seja, maximizar a medida $Q$.

Em todos os gráficos, que serão apresentados nos exemplos a seguir, as medidas que descrevemos estão em função de $\ln (\gamma)$. Num primeiro momento da análise, determina-se o valor mais adequado para o parâmetro $\gamma$, através dos gráficos fornecidos nos itens (b), (c), (d) e (e). Deseja-se baixos valores para $E\left(q_{m} / y\right)$, o que implicaria num modelo mais simples. Com relação aos gráficos (c)

e (d), espera-se também baixos valores para $\exp (H)$ e $E P$. Além disso, são desejáveis valores de $\gamma$ que produzam altos valores de $Q$. Determinado o valor de $\gamma$, iremos identificar no gráfico (a) quais preditores farão parte do modelo resultante.

Estaremos expondo os exemplos dados por Mitchell e Beauchamp (1988) como uma forma de exemplificação da teoria. Os exemplos foram baseados em dados reais de um estudo de conservação de energia. Consistem de 401 observações, que são residências familiares que participaram de um programa de conservação de energia. Foram usadas na análise dez variáveis preditoras, especificadas no artigo de estudo, sendo que a variável dependente é a economia de eletricidade (em kilowatt horas por ano).

Para análise dos dados foram feitos gráficos residuais, cálculos de $R^{2}$ e $C_{p}$ de Mallows (1973) para todos os possíveis subconjuntos de regressão, e cálculos de medidas de diagnóstico. Além disso, todas as variáveis preditoras originais foram padronizadas, subtraindo suas médias e dividindo pelos seus desvios padrões. Após padronização, as variáveis preditoras serão indicadas por $z_{1}, z_{2}, \ldots, z_{10}$. O modelo ajustado por mínimos quadrados com base nestes dados foi 


$$
\begin{aligned}
\hat{y}= & 5.068-116 z_{1}-367 z_{2}+353 z_{3}+879 z_{4}-370 z_{5}+618 z_{6}-429 z_{7}+2.812 z_{8} \\
& -452 z_{9}+433 z_{10} .
\end{aligned}
$$

Os gráficos de resíduos não mostraram nenhum problema óbvio. A magnitude dos fatores de inflação de variância (Montgomery e Peck, 1992) foram menores que 1,5. Além disso, o índice de condição da matriz $Z: Z, \theta=\frac{\lambda_{\max }}{\lambda_{\min }}$, com $\lambda_{\max }=\max \left(\lambda_{1}, \lambda_{2}, \ldots, \lambda_{q}\right), \lambda_{\min }=\min \left(\lambda_{1}, \lambda_{2}, \ldots, \lambda_{q}\right), \mathrm{e} \lambda_{1}, \lambda_{2}, \ldots, \lambda_{q}$ autovalores da matriz $Z: Z$, não indicaram nenhum problema significativo de multicolinearidade entre as variáveis preditoras, pois resultou num valor bem menor que 100 . O modelo completo não tem um alto coeficiente de explicação $(0,465)$, mas todos os coeficientes de regressão foram significativos $(\mathrm{P}<0,05)$, exceto para $\beta_{1}$, caso em que o valor de $\mathrm{P}$ é 0,497 . O submodelo com $z_{1}$ omitido foi o único para o qual $C_{p}$ foi menor que $1,1 q$. O critério $C_{p}<1,1 q$ foi utilizado para aceitação de modelos no artigo considerado.

A Figura 4.1 apresenta os gráficos propostos pelos autores para a seleção de variáveis preditoras no exemplo. 
Figura 4.1: Análise do Exemplo 1

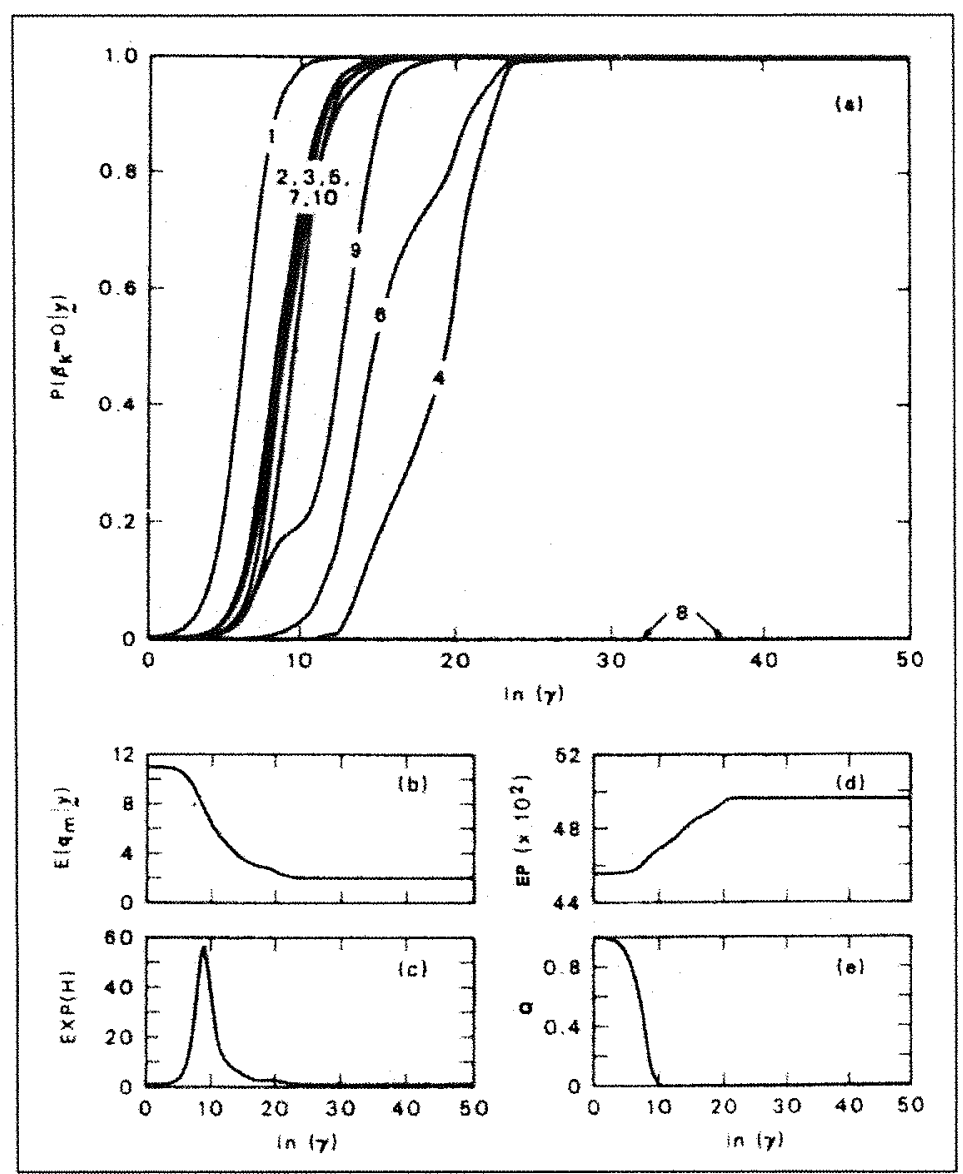

(a) Probabilidade a posteriori de $\beta_{k}=0, \operatorname{Pr}\left(\beta_{k}=0 / y\right)$;

(b) Número esperado de termos (a posteriori) no submodelo, $E\left(q_{m} / y\right)$;

(c) Antilogaritmo da perturbação (a posteriori) da distribuição do submodelo, $\exp (H)$;

(d) Erro preditivo a posteriori, $E P$;

(e) Probabilidade a posteriori da qualidade do ajuste, $Q$.

Os gráficos (d) e (e) sugerem que $\ln (\gamma)$ deveria ser menor que seis. Isto deveria ocorrer para minimizar o erro preditivo e manter uma alta probabilidade a posteriori para os submodelos que não exibem falta de ajuste. Observemos que o 
antilogaritmo da perturbação, dado no gráfico (c), começa a aumentar acentuadamente para $\ln (\gamma) \geq 6$, indicando confusão crescente na escolha do melhor submodelo. Se admitirmos $\ln (\gamma)=6$, utilizando-se o gráfico (a), observamos que o modelo ajustado conterá todos os coeficientes de regressão, e portanto, não haverá eliminação de variáveis.

Portanto, se quisermos realmente eliminar variáveis preditoras, mas mantendo qualidade do modelo, o mais apropriado seria considerarmos valores de $\ln (\gamma)$ entre 25 e 50. Desta maneira, a distribuição a posteriori é tal que $P\left(\beta_{k}=0\right)=1$ para todos os $\beta_{k}$, exceto $\beta_{0}$ e $\beta_{8}$, sugerindo o uso do submodelo com os dois termos. Neste caso, as medidas fornecidas pelos gráficos (b) e (c) estão adequadas para este submodelo, entretanto, o gráfico (d) nos mostra que esta escolha seria aceitável somente se estivermos dispostos a admitir um erro preditivo de aproximadamente 5.000, ao invés do mínimo de 4.550. Contudo, o gráfico (e) sugere que este submodelo sofre de considerável falta de ajuste. Em face de tal situação, o ideal seria considerar $\ln (\gamma) \cong 15$, pois teríamos um equilíbrio entre as duas possibilidades exibidas. Neste caso, o modelo selecionado seria $\hat{y}=f\left(z_{4}, z_{6}, z_{8}\right)$.

No segundo exemplo, foi feita uma simulação utilizando as mesmas variáveis preditoras do conjunto de dados reais, mas os valores da variável dependente foram gerados de modo que

$$
y=g(z)+\varepsilon,
$$

com

$g(z)=3.312+4.755 z_{1}+5.196 z_{2}+455 z_{3}+532 z_{4}+538 z_{5}+104 z_{6}+91 z_{7}$ 
e $\varepsilon$ é uma variável aleatória com distribuição normal com média 0 e desvio padrão 1.000. Dessa forma, os autores consideraram $\beta_{1}$ e $\beta_{2}$ grandes, $\beta_{3}, \beta_{4}$ e $\beta_{5}$ moderados, $\beta_{6}$ e $\beta_{7}$ pequenos, e $\beta_{8}, \beta_{9}$ e $\beta_{10}$ nulos.

A estimativa de mínimos quadrados de $g(z)$ com base nos dados simulados foi

$$
\begin{aligned}
\hat{y}= & 3.289+4.712 z_{1}+5.148 z_{2}+440 z_{3}+444 z_{4}+546 z_{5}+51 z_{6}+58 z_{7}-20 z_{8} \\
& +85 z_{9}+35 z_{10} .
\end{aligned}
$$

Obteve-se um valor de 0,980 para o coeficiente de explicação do modelo completo e os valores de $\mathrm{P}$ para os testes da significância dos coeficientes $\beta_{6}$ a $\beta_{10}$ foram 0,31 ; 0,$27 ; 0,73 ; 0,13$; e 0,48 , respectivamente. Havia 22 submodelos com $C_{p}$ menor que $1,1 q$, todos incluindo as variáveis preditoras $z_{1}$ a $z_{5}$. Além disso, 11 destes modelos incluíam no mínimo dois coeficientes de regressão nulos. $O$ valor mais baixo de $C_{p}$ foi 5,598, para o modelo que omitiu as variáveis preditoras $z_{6}, z_{7}, z_{8}$ e $z_{10}$.

$\mathrm{O}$ procedimento Bayesiano de seleção de variáveis para o exemplo está resumido na Figura 4.2. 
Figura 4.2: Análise do Exemplo 2

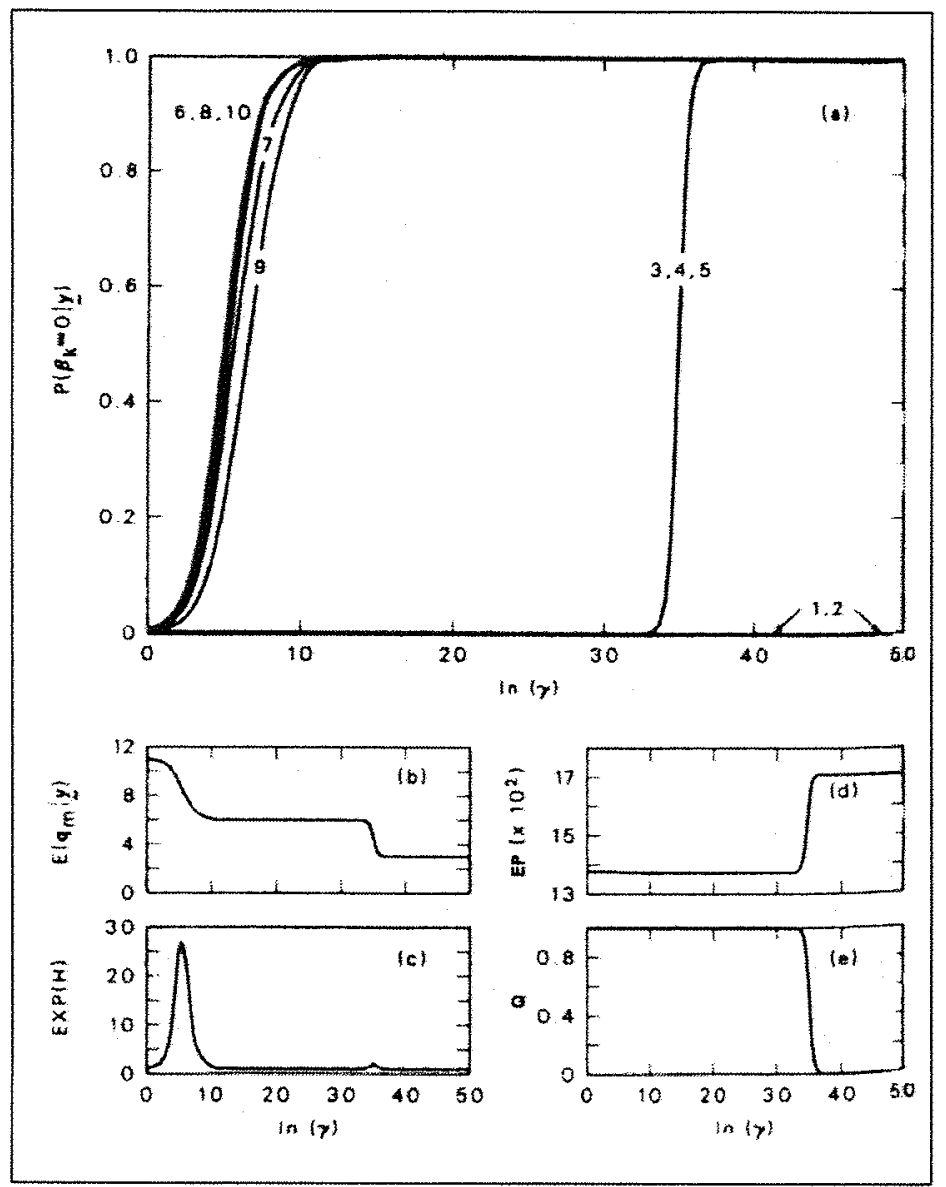

(a) Probabilidade a posteriori de $\beta_{k}=0, \operatorname{Pr}\left(\beta_{k}=0 / y\right)$;

(b) Número esperado de termos (a posteriori) no submodelo, $E\left(q_{m} / y\right)$;

(c) Antilogaritmo da perturbação (a posteriori) da distribuição do submodelo, $\exp (H)$;

(d) Erro preditivo a posteriori, $E P$;

(e) Probabilidade a posteriori da qualidade do ajuste, $Q$.

O gráfico (a) da Figura 4.2 permite ver nitidamente a separação dos preditores fortes $\left(z_{1}, z_{2}, z_{3}, z_{4}\right.$ e $\left.z_{5}\right)$ dos preditores fracos $\left(z_{6}, z_{7}, z_{8}, z_{9}\right.$ e $\left.z_{10}\right)$. Os gráficos (d) e (e) sugerem que $\ln (\gamma)$ pode ser no máximo 32, pois para valores 
maiores, o erro preditivo aumenta e a qualidade do ajuste diminui consideravelmente. O gráfico (b) mostra que para $\ln (\gamma) \cong 32$, a probabilidade a posteriori está concentrada sobre um modelo tendo seis termos, incluindo $\beta_{0}$. A partir do gráfico (a) pode-se ver que o modelo selecionado contém apenas os preditores fortes $z_{1}, z_{2}, z_{3}, z_{4}$ e $z_{5}$.

Em síntese, definimos qual o valor para $\ln (\gamma)$ através dos gráficos (b), (c), (d), e (e), de forma que haja um equilíbrio entre os critérios de seleção segundo cada gráfico. Após a escolha de $\ln (\gamma)$, buscamos o modelo selecionado no gráfico (a).

$\mathrm{Na}$ análise deste estudo de simulação, o método proposto mostrou-se bastante eficiente. Através desta análise é possível também verificar quais são os preditores fortes, ou seja, aqueles que persistem no modelo para altos valores de $\ln (\gamma)$. Isso se deve ao fato que grandes valores de $\gamma$ implicam em altas probabilidades de $\beta_{k}=0$, de acordo com a equação (4.1).

\subsection{Densidade Preditiva Bayesiana}

Nesta seção, apresentaremos o método de seleção de variáveis proposto por Geisser e Eddy $(1979,1980)$, baseado no uso de densidades preditivas.

Seja o modelo linear $\underline{Y}=Z \beta+\varepsilon$, já visto anteriormente, e o modelo associado ao conjunto de observações futuras a serem previstas $\underset{\sim}{W}=X \beta+\varepsilon^{*}$, sendo que $\underline{Y}=\left(Y_{1}, \ldots, Y_{n}\right)^{\prime}$ e $\underset{\sim}{W}=\left(W_{1}, \ldots, W_{n}\right)^{\prime}$ são respectivamente os vetores de variáveis observadas e observações futuras de dimensão $n$ e $n^{*}$. Além disso, $Z$ e $X$ são matrizes de constantes conhecidas $n \times q$ e $n^{*} \times q$, sendo que $Z$ é de posto completo. 
Tem-se $\beta=\left(\beta_{1}, \ldots, \beta_{q}\right)^{\prime}$, vetor de parâmetros de regressão desconhecidos e $\underline{\boldsymbol{\varepsilon}} \mathrm{e}$ $\varepsilon^{*}$, vetores aleatórios não observados independentes entre si de dimensões $n$ e $n^{*}$, respectivamente. Tais vetores estão distribuídos de modo que,

$$
\underline{\varepsilon} \sim N_{n}\left(0, \sigma^{2} I\right) \text { e } \varepsilon^{*} \sim N_{n^{*}}\left(0, \sigma^{2} I\right)
$$

sendo que $\sigma^{2}$ é um parâmetro positivo desconhecido. O espaço paramétrico é $\Theta=\left\{\theta=\left(\underset{\sim}{\beta} \sigma^{2}\right): \underset{\sim}{\beta} \in \Omega_{\underline{\beta}} ; \sigma^{2}>0\right\}$, sendo que $\Omega_{\underline{\beta}}$ é o espaço paramétrico para $\underline{\beta}$. Para os modelos apresentados, as densidades de $Y$ e $W$ são, respectivamente,

$$
p_{n}\left(y, Z / \underset{\sim}{\beta}, \sigma^{2}\right)=\left(2 \pi \sigma^{2}\right)^{\frac{n}{2}} \exp \left\{-\left(2 \sigma^{2}\right)^{-1}(y-Z \underset{\sim}{\beta})^{\prime}(y-Z \underset{\sim}{\beta})\right\}
$$

e

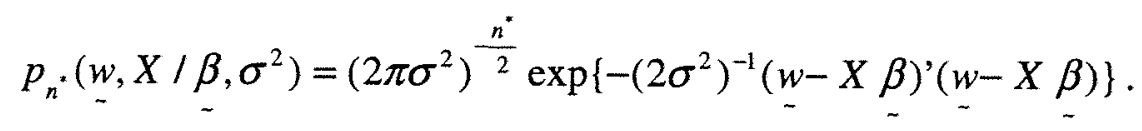

Como $\underset{\sim}{W}$ é o vetor de dados não observados, nosso objetivo é o da determinação da densidade preditiva de $\underset{\sim}{W}$. No modelo $\underset{\sim}{Y}=Z \beta+\underline{\varepsilon}$, o estimador de máxima verossimilhança do parâmetro de interesse $\theta=\left(\beta, \sigma^{2}\right)$ é $\hat{\theta}=\left(\hat{\beta}, \hat{\sigma}^{2}\right)$, com

$$
\hat{\beta}=\left(Z^{\prime} Z\right)^{-1} Z^{\prime} Y
$$

e

$$
\hat{\sigma}^{2}=\frac{(Y-Z \hat{\beta})^{\prime}(Y-Z \hat{\beta})}{n} .
$$


Se denotarmos por $\hat{\beta}_{w}$ e $\hat{\sigma}_{W}^{2}$ os estimadores de máxima verossimilhança de $\beta$ e $\sigma^{2}$, respectivamente, baseados em $(\underset{\sim}{Y, W})$, isto é, sob o modelo

$$
V=H \beta+e
$$

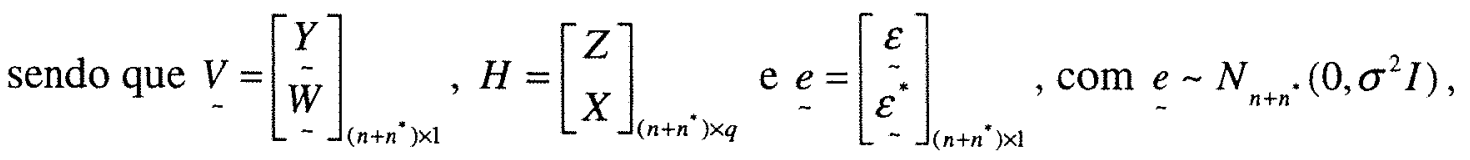

teremos então

$$
\hat{\beta}_{W}=\left(H^{\prime} H\right)^{-1} H^{\prime} V
$$

e

$$
\hat{\sigma}_{W}^{2}=\frac{\left(V-H \hat{\beta}_{W}\right)^{\prime}\left(V-H \hat{\beta}_{W}\right)}{-}
$$

Essas quantidades serão utilizadas para obter a densidade preditiva de $W$.

Neste caso, a densidade preditiva bayesiana para as observações futuras $\underset{\sim}{w}$, baseada no vetor de dados observados $y$, foi obtida por Levy e Perng (1984), e é da forma

$$
r(\underset{w}{w} X / \underset{\sim}{y}, Z)=\int_{\theta \in \Theta} f\left(\underset{\sim}{w}, X / \underset{\sim}{y, Z} \underset{\sim}{\beta}, \sigma^{2}\right) f\left(\underset{\sim}{\beta}, \sigma^{2} / \underset{\sim}{y}, Z\right) d \underset{\sim}{\beta d} d \sigma^{2}
$$

sendo que 
$f\left(\underset{w}{w} X / \underset{\sim}{y}, \boldsymbol{\sim}, \sigma^{2}\right)$ é a densidade condicional de $\underset{w}{ }$ dado $y \mathrm{e}$ $f\left(\beta, \sigma^{2} / \underset{\sim}{y}, Z\right)$ é a densidade a posteriori de $\left(\beta, \sigma^{2}\right)$ dado $y$.

Para os parâmetros $\underset{\sim}{\beta}$ e $\sigma^{2}$, foi utilizada a densidade a priori $f\left(\underset{\sim}{\beta}, \sigma^{2}\right) \propto \frac{1}{\sigma^{2}}$ e com isto, a densidade a posteriori de $\left(\beta, \sigma^{2}\right)$ dado $y$ é calculada como

$$
f\left(\beta, \sigma^{2} / y, Z\right)=\frac{f\left(\beta, \sigma^{2}\right) p_{n}\left(y, Z / \beta, \sigma^{2}\right)}{\int_{\theta \in \Theta} f\left(\beta, \sigma^{2}\right) p_{n}\left(y, Z / \beta, \sigma^{2}\right) d \beta d \sigma^{2}} .
$$

Como as variáveis aleatórias $Y$ e $W$ são independentes dado $\theta$, temos que

$$
f\left(\underset{w}{w}, X / \underset{\sim}{y}, Z, \underset{\sim}{\beta}, \sigma^{2}\right)=f\left(\underset{w}{w}, X / \beta, \sigma^{2}\right)=p_{n^{*}}\left(\underset{\sim}{w}, X / \beta, \sigma^{2}\right) .
$$

Substituindo-se na expressão de $r(w, X / y, Z)$, teremos que a Densidade Preditiva Bayesiana para $W$ é dada por

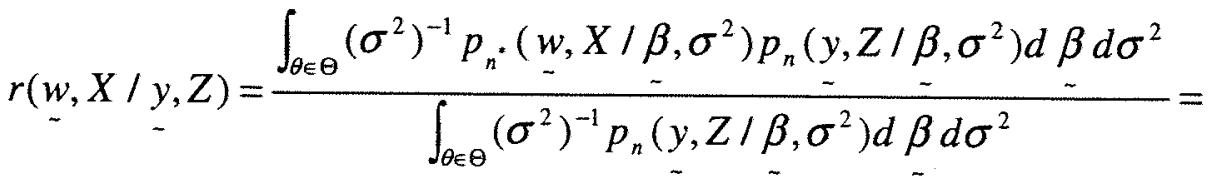

$$
\begin{aligned}
& =\frac{\int_{\theta \in \Theta}\left(\sigma^{2}\right)^{-1}\left(2 \pi \sigma^{2}\right)^{\frac{n+n^{*}}{2}} \exp \left\{-\left(2 \sigma^{2}\right)^{-1}\left[(y-Z \beta)^{\prime}(y-Z \beta)+(w-X \beta)^{\prime}(w-X \beta)\right]\right\} d \underline{\beta} d \sigma^{2}}{\int_{\theta \in \Theta}\left(\sigma^{2}\right)^{-1}\left(2 \pi \sigma^{2}\right)^{-\frac{n}{2}} \exp \left\{-\left(2 \sigma^{2}\right)^{-1}(y-Z \beta)^{\prime}(y-Z \beta)\right\} d \beta d \sigma^{2}}
\end{aligned}
$$




$$
\begin{aligned}
& =\frac{\int_{\theta \in \Theta}\left(\sigma^{2}\right)^{-1}\left(2 \pi \sigma^{2}\right)^{\frac{n+n^{*}}{2}} \exp \left\{-\left(2 \sigma^{2}\right)^{-1}\left[\begin{array}{c}
y-Z \beta \\
\tilde{w}-X \tilde{\beta}
\end{array}\right]\left[\begin{array}{c}
y-Z \beta \\
\tilde{w}-X \tilde{\beta} \\
-
\end{array}\right]\right\} d \beta d \sigma^{2}}{\int_{\theta \in \Theta}\left(\sigma^{2}\right)^{-1}\left(2 \pi \sigma^{2}\right)^{-\frac{n}{2}} \exp \left\{-\left(2 \sigma^{2}\right)^{-1}(y-Z \beta)^{\prime}(y-Z \beta)\right\} d \beta d \sigma^{2}} \\
& =\frac{\int_{\theta \in \Theta}\left(\sigma^{2}\right)^{-1}\left(2 \pi \sigma^{2}\right)^{-\frac{n+n^{*}}{2}} \exp \left\{-\left(2 \sigma^{2}\right)^{-1}(\nu-H \beta)^{\prime}(\nu-H \beta)\right\} d \beta d \sigma^{2}}{\int_{\theta \in \Theta}\left(\sigma^{2}\right)^{-1}\left(2 \pi \sigma^{2}\right)^{-\frac{n}{2}} \exp \left\{-\left(2 \sigma^{2}\right)^{-1}(y-Z \beta)^{\prime}(y-Z \beta)\right\} d \beta d \sigma^{2}} .
\end{aligned}
$$

Após cálculos (Rojas, 2000, Apêndice A.2) obtém-se a densidade Preditiva Bayesiana para $w$

$$
\begin{aligned}
& r(\underset{\sim}{w}, X / \underset{\sim}{y}, Z)=\frac{\Gamma\left(\frac{n+n^{*}-q}{2}\right)}{[\pi(n-q)]^{\frac{n^{*}}{2}} \Gamma\left(\frac{n-q}{2}\right)}\left|n \hat{\sigma}^{2} \frac{\left(I+\underset{\sim}{X}\left(Z^{\prime} Z\right)^{-1} X^{\prime}\right)}{(n-q)}\right|^{\frac{1}{2}} \times \\
& \times\left\{1+\frac{1}{(n-q)}(\underline{w}-X \hat{\beta})^{\prime}\left[n \hat{\sigma}^{2} \frac{\left(I+\underset{X}{X}\left(Z^{\prime} Z\right)^{-1} X^{\prime}\right)}{(n-q)}\right]^{-1}(w-X \hat{\beta})\right\}^{-\frac{n+n^{*}-q}{2}} .
\end{aligned}
$$

Veremos a seguir como selecionar um modelo de regressão linear múltipla através do uso de densidades preditivas. Vamos supor que queremos prever uma única observação. Assim, admitimos que $X=x$, ou seja, $X$ é um vetor linha de dimensão $q$. Desta forma, altera-se apenas o vetor aleatório $\varepsilon^{*}$, que terá dimensão $1 \times 1$, e assim segue que $\varepsilon^{*} \sim N\left(0, \sigma^{2}\right)$. 
Para os modelos definidos inicialmente e com a metodologia introduzida, Geisser (1965) obteve a densidade preditiva de $W$, vetor de observações futuras de dimensão $n^{*}$, sendo que a densidade a priori para $\left(\beta, \sigma^{2}\right)$ é $f\left(\beta, \sigma^{2}\right)$ proporcional a $\sigma^{-1}$

Com isso, a densidade preditiva de $W$, unidimensional é dada por

$$
f(w / y, x, Z)=\left(\frac{c}{\pi a^{2}}\right)^{\frac{1}{2}} \frac{\Gamma\left(\frac{1}{2}(n-q)\right)}{\Gamma\left(\frac{1}{2}(n-q-1)\right)}\left[1+\frac{c(w-x \hat{\beta})^{2}}{a^{2}}\right]^{-\frac{1}{2}(n-q)},
$$

$\operatorname{com} c=I-\underset{\sim}{x}\left(Z^{\prime} Z\right)^{-1} x_{\sim}^{\prime}, a^{2}=(y-Z \hat{\beta})^{\prime}(y-Z \hat{\beta})$.

Considerando $q$ variáveis preditoras, a densidade preditiva para uma observação $y_{i}$ retirada do modelo, dado as observações que ficaram $y_{(i)}=\left(y_{1}, \ldots, y_{i-1}, y_{i+1}, \ldots, y_{n}\right)^{\prime}$ será

$$
f\left(y_{i} / y_{(i)}, Z_{i}, Z_{(i)}\right)=\left(\frac{c_{i}}{\pi a_{(i)}^{2}}\right)^{\frac{1}{2}} \frac{\Gamma\left(\frac{1}{2}(n-q-1)\right)}{\Gamma\left(\frac{1}{2}(n-q-2)\right)}\left[1+\frac{c_{i}\left(y_{i}-Z_{i} \hat{\beta}_{(i)}\right)^{2}}{a_{(i)}^{2}}\right]^{-\frac{1}{2}(n-q-1)},
$$

para $i=1,2, \ldots, n$, com

$c_{i}=I-Z_{i}\left(Z^{\prime} Z\right)^{-1} Z_{i}^{\prime}$,

$a_{(i)}^{2}=\left(y_{(i)}-Z_{(i)} \hat{\beta}_{(i)}\right)^{\prime}\left(y_{(i)}-Z_{(i)} \hat{\beta}_{(i)}\right)$,

$\hat{\beta}_{(i)}=\left(Z_{(i)}^{\prime} Z_{(i)}\right)^{-1} Z_{(i)}^{\prime} y_{(i)}$, 


$$
\begin{aligned}
& Z=\left(Z_{1}^{\prime}, \ldots, Z_{n}^{\prime}\right)^{\prime}, \\
& Z_{(i)}=\left(Z_{1}^{\prime}, \ldots, Z_{i-1}^{\prime}, Z_{i+1}^{\prime}, \ldots, Z_{n}^{\prime}\right)^{\prime} \mathrm{e} \\
& Z_{i}=\left(z_{i 1}, \ldots, z_{i q}\right) .
\end{aligned}
$$

Geisser (1975) propõe o uso da medida de verossimilhança, definida como

$$
L=\prod_{i=1}^{n} f\left(y_{i} / y_{(i)}, Z_{i}, Z_{(i)}\right)=\prod_{i=1}^{n}\left(\frac{c_{i}}{\pi a_{(i)}^{2}}\right)^{\frac{1}{2}} \frac{\Gamma\left(\frac{1}{2}(n-k-1)\right)}{\Gamma\left(\frac{1}{2}(n-k-2)\right)}\left[1+\frac{c_{i}\left(y_{i}-Z_{i} \hat{\beta}_{(i)}\right)^{2}}{a_{(i)}^{2}}\right]^{-\frac{1}{2}(n-k-1)},
$$

com $k=1,2, \ldots, q$, sendo que $k$ representa o número de variáveis preditoras no modelo. Assim, a medida de verossimilhança preditiva associada ao $m$-ésimo modelo ficará

$$
L_{m}=\prod_{i=1}^{n} f\left(y_{i} / y_{(i)}, Z_{i}, Z_{(i)}\right)=\prod_{i=1}^{n}\left(\frac{c_{i}}{\pi a_{(i)}^{2}}\right)^{\frac{1}{2}} \frac{\Gamma\left(\frac{1}{2}\left(n-k_{m}-1\right)\right)}{\Gamma\left(\frac{1}{2}\left(n-k_{m}-2\right)\right)}\left[1+\frac{c_{i}\left(y_{i}-Z_{i}^{m} \hat{\beta}_{(i)}\right)^{2}}{a_{(i)}^{2}}\right]^{-\frac{1}{2}\left(n-k_{m}-1\right)},
$$

para $m=1,2, \ldots, 2^{q}-1, k_{m}$ é o número de variáveis preditoras no $m$-ésimo modelo e $Z_{i}^{m}$ é o vetor de valores das variáveis preditoras presentes no modelo considerado associado a $y_{i}$.

Vamos supor que $M_{m}: M_{1}, \ldots, M_{2^{q}-1}$ são todos os possíveis modelos. O critério apresentado consiste em escolher o modelo $M_{m^{*}}$ tal que $L_{m^{*}}$ é o maior de todos os $L_{m}, m=1,2, \ldots, 2^{q}-1$, ou seja, $M_{m}$. representa o modelo que maximiza $L_{m}$.

Para exemplificar o método descrito, Geisser e Eddy (1980) utilizaram o conjunto de dados de Hald (Draper e Smith (1998)). Os autores calcularam os 
valores de $\log L_{m}$ para os quinze possíveis modelos de regressão com intercepto. Após análise dos valores, constatou-se que o modelo que contém as variáveis $Z_{1}$ e $Z_{2}$ possui o maior valor de $L_{m}$. Verificou-se ainda que os critérios $\log L_{m}$ e $C_{p}$ são assintoticamente equivalentes quando a variável resposta $Y$ tem distribuição normal.

Além disso, é importante salientar que o modelo selecionado pelo critério proposto coincidiu com o modelo obtido nos procedimentos $C_{p}$ e Stepwise (Draper e Smith (1998)).

\subsection{Amostrador de Gibbs}

O método de seleção de variáveis que iremos descrever a seguir está apresentado com detalhes em George e McCulloch (1993). O procedimento consiste em usar considerações probabilísticas para selecionar submodelos. O procedimento foi chamado pelos autores de investigação aleatória de seleção de variáveis (IASV). A IASV é baseada na estrutura de modelo linear completo Bayesiano hierárquico, definido como uma mistura de normais, onde variáveis indicadoras são usadas para apontar os subconjuntos mais promissores.

$\mathrm{O}$ amostrador de Gibbs é usado para diminuir a carga computacional. $\mathrm{O}$ objetivo é identificar indiretamente a partir da distribuição a posteriori do conjunto de variáveis indicadoras, os subconjuntos de variáveis preditoras possíveis para o modelo. Os subconjuntos de preditores com probabilidade a posteriori alta têm mais chance de entrar no modelo e estes podem ser identificados por aparecer com alta frequiência na amostragem de Gibbs. Com esta técnica, é possível evitar o problema de calcular as probabilidades a posteriori de todos os $2^{q}$ subconjuntos.

A investigação é controlada por diversos parâmetros decisivos, que podem ser pré-especificados pelo usuário. O usuário pode manipular as diferentes pré- 
especificações de forma apropriada para selecionar variáveis de acordo com um objetivo. Um dos possíveis objetivos é a busca por um modelo econômico, que não aumente drasticamente o erro de previsão e não provoque a eliminação de variáveis importantes.

A literatura Bayesiana para seleção de variáveis trabalha com duas linhas de pesquisa. A primeira está descrita na Seção 4.2 e usa o modelo de Bayes hierárquico para identificar as variáveis que têm potencial. Já a segunda linha refere-se ao amostrador de Gibbs. Iremos definir o modelo hierárquico que servirá como a base para a IASV. Em seguida, mostraremos como este modelo hierárquico pode ser usado para identificar a maior parte dos modelos de regressão que têm potencial e, portanto, como a investigação via amostrador de Gibbs pode eficientemente identificar subconjuntos promissores.

Com intuito de extrair informação relevante para selecionar variáveis, considere a estrutura de regressão

$$
\underset{\sim}{Y I} \underset{\sim}{\beta} \sigma^{2} \sim N_{n}\left(Z \underset{\sim}{\beta}, \sigma^{2} I\right)
$$

sendo que $\underset{\sim}{Y}$ é um vetor aleatório de dimensão $n, Z=\left[Z_{1}, \ldots, Z_{q}\right]$ é a matriz de planejamento $n \times q, \beta=\left(\beta_{1}, \ldots, \beta_{q}\right)$ ' e $\sigma^{2}$ é um escalar. Tanto $\beta$ quanto $\sigma^{2}$ são considerados desconhecidos.

O modelo hierárquico que iremos apresentar é tal que cada componente de $\beta$ está modelada como uma mistura de duas distribuições normais com variâncias diferentes. A partir das variáveis indicadoras

$$
\gamma_{k}=0 \text { ou } \gamma_{k}=1, \text { para } k=1, \ldots, q
$$


os autores representaram a mistura de normais por

$$
\beta_{k} / \gamma_{k} \sim\left(1-\gamma_{k}\right) N\left(0, \tau_{k}^{2}\right)+\gamma_{k} N\left(0, c_{k}^{2} \tau_{k}^{2}\right)
$$

$\mathrm{e}$

$$
P\left(\gamma_{k}=1\right)=1-P\left(\gamma_{k}=0\right)=p_{k}
$$

Quando $\quad \gamma_{k}=0$, tem-se $\beta_{k} / \gamma_{k}=0 \sim N\left(0, \tau_{k}^{2}\right)$, e quando $\gamma_{k}=1$, tem-se $\beta_{k} / \gamma_{k}=1 \sim N\left(0, c_{k}^{2} \tau_{k}^{2}\right)$. Inicialmente, fixa-se $\tau_{k}$ baixo de modo que se $\gamma_{k}=0$, o valor de $\beta_{k}$ seria provavelmente tão pequeno que poderia ser seguramente estimado por sua média que é 0 . Já, se $\gamma_{k}=1$, então para $c_{k}$ grande, maior que um, uma estimativa não nula de $\beta_{k}$ deveria provavelmente ser incluída no modelo final.

Baseada nesta interpretação, $p_{k}$ pode ser encarado como a probabilidade a priori que $\beta_{k}$ venha apresentar uma estimativa não nula, ou equivalentemente que a variável preditora $Z_{k}$ deva ser incluída no modelo.

Uma construção semelhante foi utilizada na Seção 4.2 , no entanto, naquela abordagem, foi especificada uma probabilidade a priori de $\beta_{k}=0$, o que não ocorre aqui.

Para se obter (4.7) como uma priori para $\beta_{k} / \gamma_{k}$, os autores usaram a priori normal multivariada para $\beta$, dada por

$$
\beta / \gamma \sim N_{q}\left(0, D_{\gamma} R D_{\gamma}\right)
$$


sendo que $\gamma=\left(\gamma_{1}, \gamma_{2}, \ldots, \gamma_{q}\right), R$ é a matriz de correlação a priori de $\beta$ e $D_{\gamma}$ é dado por

$$
D_{\gamma} \equiv \operatorname{diag}\left[a_{1} \tau_{1}, \ldots, a_{q} \tau_{q}\right]
$$

com

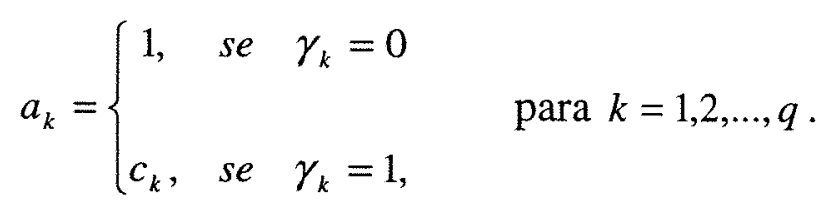

Considera-se $\tau_{k}$ pequeno (maior que zero) e $c_{k}$ grande (sempre maior que um), de forma que em (4.7), os $\beta_{k}$ para os quais $\gamma_{k}=0$ tenderão a concentrar-se ao redor de 0 , enquanto aqueles $\beta_{k}$ para os quais $\gamma_{k}=1$ tenderão a se dispersar.

Por fim, deve-se supor uma distribuição a priori para a variância residual $\sigma^{2}$. Para tal finalidade, foi utilizada a distribuição gama inversa

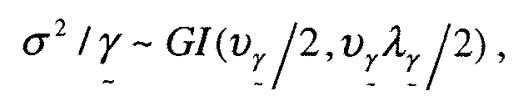

que é equivalente a termos $v_{\underline{\gamma}} \lambda_{\underline{\gamma}} / \sigma^{2} \sim \chi_{v_{\underline{\gamma}}}^{2}$. Observe que $v_{\underline{\gamma}}$ e $\lambda_{\gamma}$ são parâmetros que podem depender de $\gamma$ para incorporar dependência entre $\beta$ e $\sigma^{2}$. A variável aleatória $W$ tem distribuição Gama Inversa, $G I(\alpha, \beta)$, se sua função densidade de probabilidades é dada por 


$$
p(w / \alpha, \beta)=\frac{\beta^{\alpha}}{\Gamma(\alpha)} w^{-(\alpha-1)} e^{-\beta / w}, w>0, \text { para } \alpha, \beta>0
$$

A densidade da Gama Inversa pode ser encontrada em Bernardo e Smith (1994).

Escrever o modelo linear normal (4.6) como mistura de modelos hierárquicos tem como finalidade a obtenção da distribuição a posteriori marginal $f(\gamma / Y) \propto f(Y / \gamma) f(\gamma)$, que contém a informação necessária para seleção de variáveis. Com o propósito de selecionar variáveis, $f(\gamma)$ deve ser interpretada como a probabilidade a priori, especificada pelo estatístico, de que as variáveis $Z_{k}$ correspondendo aos valores de $\gamma_{k}=1$ (e somente elas) sejam incluídas no modelo final.

Fixado $\gamma$, temos que a distribuição a priori de $\beta$ é uma mistura finita de prioris normais multivariadas. A densidade a posteriori $f(\gamma / Y)$ fornece as probabilidades de cada possível valor de $\gamma$, dentre os $2^{q}$ possíveis, uma vez observado o conjunto de dados. Identificando cada $\gamma$ com um submodelo, sendo que $\left(\gamma_{k}=1\right) \Leftrightarrow\left(Z_{k}\right.$ está incluído), os $\gamma$ com alta probabilidade a posteriori, ou seja, valores altos de $f(\gamma / Y)$, identificam os submodelos mais coerentes com os dados e a informação a priori baseada na crença subjetiva do estatístico. Assim, $f(\gamma / Y)$ nos proporciona informação sobre modelos que têm características adequadas para serem investigadas. O valor de $f(\gamma / Y)$ depende das escolhas para as constantes $\tau_{k} \mathrm{e}$ $c_{k}$, para $k=1,2, \ldots, q$, usada em $D_{\gamma}$, da matriz de correlação a priori $R$, e das escolhas de $v_{\gamma}$ e $\lambda_{\gamma}$. Portanto, iremos mostrar a seguir como lidar com tais escolhas. 
- A escolha de $f(\gamma)$ deve incorporar toda informação a priori disponível sobre a importância das variáveis $Z_{1}, Z_{2}, \ldots, Z_{q}$. Além disso, uma escolha razoável parte da independência dos $\gamma_{k}$ 's com distribuição marginal (4.8), de forma que

$$
f(\gamma)=\prod_{k=1}^{q} p_{k}^{\gamma_{k}}\left(1-p_{k}\right)^{\left(1-\gamma_{k}\right)}, \text { para } \underset{\sim}{\gamma}=\left(\gamma_{1}, \gamma_{2}, \ldots, \gamma_{k}\right)
$$

A priori uniforme $f(\gamma) \equiv 2^{-q}$ é o caso especial de (4.12), quando todos os $Z_{k}$ 's têm mesma chance de serem incluídos, ou seja, $p_{k}=\frac{1}{2}, k=1,2, \ldots, q$. Outra alternativa seria dar pesos aos modelos segundo o seu tamanho, usando $f(\underset{\sim}{\gamma})=w_{|\gamma|}(\mid \underset{-1}{q})^{-1}$, sendo que $|\gamma|$ é o número de componentes iguais a $1 \mathrm{em} \gamma$ e $w_{|\gamma|}$ seria a probabilidade a priori de um modelo de tamanho $|\gamma|$. Com $w_{|\gamma|}$ grande para $|\gamma|$ pequeno, pode-se atribuir mais peso aos modelos econômicos.

- A escolha de $\tau_{k}$ para (4.7) e (4.10) é obtida admitindo que se $\beta_{k} \sim N\left(0, \tau_{k}^{2}\right)$, ou seja, se $\gamma_{k}=0$, então $\beta_{k}$ pode ser seguramente substituído por 0 . Isto ocorre porque nessa distribuição, $\left|\beta_{k}\right| \leq 3 \tau_{k}$ com alta probabilidade. Assim, o pesquisador poderia fixar $3 \tau_{k}$ como máximo do valor para o qual, na prática, $\beta_{k}$, poderia ser considerado insignificante. Tal escolha, no entanto, pode ser inviável, já que geralmente não é conhecido o efeito de cada variável no modelo final. 
- Nas expressões (4.7) e (4.10), a escolha de $c_{k}$, sempre maior que um, em $\beta_{k} \sim N\left(0, c_{k}^{2} \tau_{k}^{2}\right)$, proporcionaria uma estimativa não nula para $\beta_{k}$, e conseqüentemente, esta estimativa deveria ser incluída no modelo final. Como auxílio na escolha de $c_{k}$, pode ser útil observar que as densidades de $N\left(0, \tau_{k}^{2}\right)$ e $N\left(0, c_{k}^{2} \tau_{k}^{2}\right)$ se interceptam em $\beta_{k}=\xi\left(c_{k}\right) \tau_{k} \operatorname{com} \xi\left(c_{k}\right)=\sqrt{2\left(\log c_{k}\right) c_{k}^{2} /\left(c_{k}^{2}-1\right)}$. Isto implica que a densidade $N\left(0, c_{k}^{2} \tau_{k}^{2}\right)$ será maior que a densidade $N\left(0, \tau_{k}^{2}\right)$ se e somente se $\left|\beta_{k}\right|>\xi\left(c_{k}\right) \tau_{k}$. Através de cálculos, é possível verificar que o ponto de interseção aumenta muito lentamente com $c_{k}$. Além disso, também pode ser útil observar que $c_{k}$ é a razão das ordenadas das distribuições $N\left(0, \tau_{k}^{2}\right)$ e $N\left(0, c_{k}^{2} \tau_{k}^{2}\right)$ para $\beta_{k}=0$. Assim, a constante $c_{k}$ pode ser interpretada como a razão de chances sob as duas distribuições de que $Z_{k}$ seja excluída quando $\beta_{k}$ estiver muito próximo de 0 .

- A matriz $R$ é a matriz de correlações da distribuição de $\beta$ dado $\gamma$. Quando $R=I$, admite-se que os componentes de $\beta$ são independentes sob a densidade $f(\beta / \gamma)$

- Normalmente, a escolha de $v_{\gamma}$ e $\lambda_{\gamma}$ para (4.11) é tal que estes valores serão constantes, isto é, $v_{\gamma} \equiv v$ e $\lambda_{\gamma} \equiv \lambda$ ou dependerão no máximo de $\gamma$ somente através de $|\gamma|$, que é o número de componentes não nulos de $\gamma$. Usualmente, adota-se $v_{\gamma} \equiv 0$ e $\lambda_{\gamma}$ qualquer, que representaria a falta de conhecimento sobre $\sigma^{2}$. 
Como já comentamos, ao invés de calcular todas as $2^{q}$ possíveis probabilidades a posteriori em $f(\gamma / Y)$, o procedimento IASV usa o amostrador de Gibbs para gerar uma seqüência

$$
\gamma^{1}, \ldots, \gamma^{m}
$$

que em muitos casos converge em distribuição rapidamente para $\gamma$, com distribuição $f(\gamma / Y)$. De modo geral, esta sequiência pode ser obtida eficientemente e de maneira mais fácil e rápida do que se calculássemos a distribuição a posteriori fornecida $f(\gamma / Y)$. Além disso, é fundamental observar que a seqüência (4.13) conterá com alta probabilidade exatamente a informação relevante para seleção de variáveis. Isto ocorre porque aqueles $\gamma$ com as mais altas probabilidades, aparecerão mais freqüentemente, e assim serão facilmente identificados. Os $\gamma$ que não aparecerem ou aparecerem com menor freqüência, podem ser ignorados.

O procedimento de investigação usará o amostrador de Gibbs para gerar uma seqüência de Gibbs auxiliar

$$
\beta^{0}, \sigma^{0}, \gamma^{0}, \beta^{1}, \sigma^{1}, \gamma^{1}, \ldots, \beta^{j}, \sigma^{j}, \gamma^{j}, \ldots
$$

Com exceção dos valores $\beta^{0}, \sigma^{0}$, e $\gamma^{0} \equiv(1,1, \ldots, 1)$, que são pré-definidos, os valores seguintes, para as iterações $1, \ldots, j$, são determinados sucessivamente como valores simulados de acordo com o procedimento de amostragem iterativo, descrito a seguir. 
Desta forma a sequiência de Gibbs, para cada iteração, $1, \ldots, j$, é obtida através dos seguintes passos

$\left.1^{\circ}\right)$ Seja $\beta^{0}$ a estimativa de mínimos quadrados no modelo completo. Obtém-se o vetor de coeficientes $\beta^{j}$ através de amostragem aleatória com

$$
\beta^{j} \sim f\left(\beta^{j} / \underline{\sim}, \sigma^{j-1}, \gamma^{j-1}\right)=N_{q}\left(A_{\gamma^{j-1}}\left(\sigma^{j-1}\right)^{-2} Z Z \beta^{0}, A_{\gamma^{j-1}}\right),
$$

sendo que $A_{\gamma^{j-1}}=\left(\left(\sigma^{j-1}\right)^{-2} Z^{\prime} Z+D_{y^{j-1}}^{-1} R^{-1} D_{\gamma^{j-1}}^{-1}\right)^{-1}$. A derivação desta densidade pode ser feita de forma rápida utilizando-se os resultados presentes em Lindley [1983, pág. 35]. Note que $D_{\gamma}^{-1}=\operatorname{diag}\left[\left(a_{1} \tau_{1}\right)^{-1}, \ldots,\left(a_{q} \tau_{q}\right)^{-1}\right]$ é facilmente calculado.

$2^{\circ}$ ) Em seguida, obtém-se a variância $\sigma^{j}$ amostrando a partir de

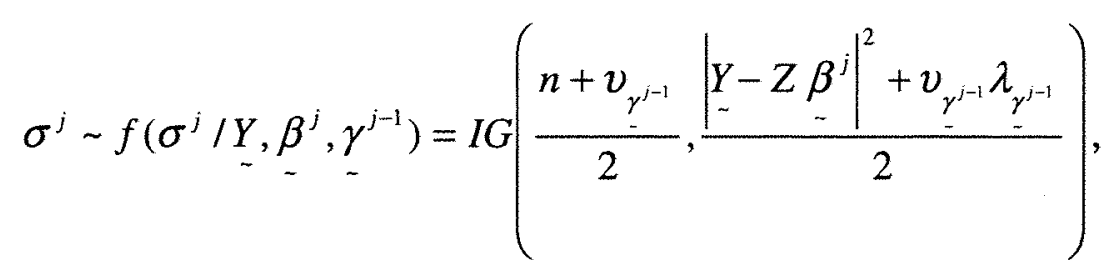

que é a distribuição inversa da gama, obtida a partir de (4.11) e atualizada utilizando-se o resultado fornecido no segundo passo.

$3^{\circ}$ ) Finalmente, o vetor $\gamma^{j}$ é obtido amostrando componente a componente consecutivamente, a partir da distribuição condicional 


$$
\gamma_{k}^{j} \sim f\left(\gamma_{k}^{j} / \underline{\sim}_{-} \beta^{j}, \sigma^{j}, \gamma_{(k)}^{j}\right)
$$

sendo que $\gamma_{(k)}^{j}=\left(\gamma_{1}^{j}, \ldots, \gamma_{k-1}^{j}, \gamma_{k+1}^{j-1}, \ldots, \gamma_{q}^{j-1}\right), k=2, \ldots, q$. Observa-se que a distribuição (4.15) não depende de $\underline{Y}$, coincidindo com $f\left(\gamma_{k}^{j} / \beta^{j}, \sigma^{j}, \gamma_{(k)}^{j}\right)$. Esta importante simplificação reduz o trabalho computacional e permite a convergência rápida da subsequiência (4.13). A não dependência de (4.15) em $Y$ é consequiência da estrutura do modelo hierárquico proposto.

Verifica-se que para cada $k$, a distribuição (4.15) é Bernoulli com probabilidade

$$
P\left(\gamma_{\underline{\sim}}^{j}=1 / \beta_{\sim}^{j}, \sigma^{j}, \gamma_{(k)}^{j}\right)=\frac{a}{a+b},
$$

sendo que

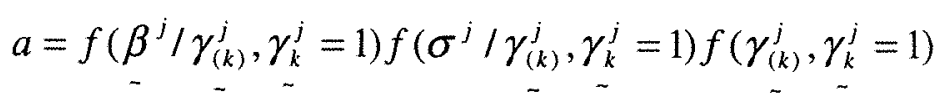

$\mathrm{e}$

$$
b=f\left(\beta^{j} / \gamma_{(k)}^{j}, \gamma_{k}^{j}=0\right) f\left(\sigma^{j} / \gamma_{(k)}^{j}, \gamma_{k}^{j}=0\right) f(\underbrace{j}_{(k)}, \gamma_{k}^{j}=0)
$$

É necessário ressaltar que na priori de $\gamma$ apresentada em (4.12), quando os parâmetros da priori em $\sigma$ em (4.11) são constantes, ou seja, $v_{\gamma} \equiv v$ e $\lambda_{\gamma} \equiv \lambda$, a equação (4.16) pode ser obtida de forma simples como 


$$
a=f\left(\beta^{j} / \gamma_{(k)}^{j}, \gamma_{k}^{j}=1\right) p_{k}
$$

e

$$
b=f\left(\beta^{j} / \gamma_{(k)}^{j}, \gamma_{k}^{j}=0\right)\left(1-p_{k}\right)
$$

Repetindo-se os passos 1, 2 e 3 várias vezes, a seqüência de Gibbs (4.14) é obtida. Assim, será possível identificar os subconjuntos de preditores, $Z_{1}, \ldots, Z_{q}$, com potencial para entrar no modelo através da seqüência $\gamma_{-}^{1}, \ldots, \gamma^{m}$ correspondente. Valores de $\gamma$ com as freqüências mais altas, terão a maior probabilidade a posteriori $f(\gamma / Y)$, e serão analisados. Se não há altas freqüências, então conclui-se que $m$ é muito pequeno ou os dados contém pouca informação para discriminar os modelos. Recomenda-se escolher $m$ o maior possível para atenuar qualquer viés nas estimativas das freqüências em (4.13). Verifica-se que com o aumento do comprimento da subsequiência $\gamma^{1}, \ldots, \gamma^{m}$, a distribuição empírica de $\gamma$ converge para a distribuição a posteriori $f(\gamma / Y)$.

Pode-se também considerar a freqüência marginal de $\gamma_{k}=1$ como evidência para inclusão de $Z_{k}$. Porém, esta aproximação pode ser enganosa, a não ser que haja pouca ou nenhuma correlação entre as variáveis preditoras. A análise de inclusão dessa variável deve ser feita em conjunto com as demais variáveis preditoras.

A convergência de (4.13) pode ser muito lenta em certas situações por exemplo, quando se escolhe $\tau_{k}$ muito pequeno e $c_{k}$ muito grande. Neste caso, a 
priori para $\beta_{k}$ é próxima de uma mistura com probabilidade não nula em zero e os outros valores distribuídos uniformemente num intervalo que não contém o zero.

Para exemplificar, apresentaremos dois exemplos simulados, discutidos pelos autores. O primeiro, trata de um problema com cinco variáveis preditoras e 60 observações. Considerou-se dois casos que identificaremos por (a) e (b):

a) Os preditores $Z_{1}, Z_{2}, \ldots, Z_{5}$ são vetores aleatórios independentes de dimensão $n=60$, com distribuição $N_{60}(0, I)$. A variável dependente foi gerada segundo o modelo

$$
Y=Z_{4}+1,2 Z_{5}+\mathcal{E}
$$

sendo que $\underset{\sim}{\mathcal{E}} \sim N_{60}\left(0, \sigma^{2} I\right)$ com $\sigma=2,5$. Assim, os coeficientes de regressão desse modelo são $\beta^{\prime}=(0 ; 0 ; 0 ; 1 ; 1,2)$. As estimativas de mínimos quadrados para estes dados foram $\hat{\beta}^{\prime}=(0,03 ;-0,45 ; 0,23 ; 0,84 ; 1,29)$, com erros padrão $\hat{\sigma}_{\beta}{ }^{\prime}=(0,35 ; 0,40$; $0,36 ; 0,31 ; 0,33)$ e $\hat{\sigma}=2,58$.

b) Este caso ilustra como a investigação aleatória de seleção de variáveis se comporta quando há presença de extrema multicolinearidade. O caso é idêntico ao apresentado em (a), exceto que $Z_{3}$ é substituído por $Z_{3}^{*}=Z_{5}+0,15 Z$ sendo que $\underset{\sim}{Z} \sim N_{60}(0, I)$, produzindo assim correlação entre $Z_{3}$ e $Z_{5}$ igual 0,989. Neste caso, as estimativas de mínimos quadrados são $\hat{\beta}^{\prime}=(0,01 ;-0,38 ; 0,34 ; 0,83 ; 0,95)$, com erros padrão $\hat{\sigma}_{\beta}{ }^{\prime}=(0,35 ; 0,39 ; 2,33 ; 0,31 ; 2,35)$ e $\hat{\sigma}=2,59$. Embora as estimativas 
dos coeficientes sejam muito próximas dos obtidos em (a), os erros padrão para $\hat{\beta}_{3} \mathrm{e}$ $\hat{\beta}_{5}$ tiveram um aumento significativo devido à multicolinearidade induzida.

Aplicando o procedimento IASV para ambos os casos com priori uniforme $f(\gamma) \equiv\left(\frac{1}{2}\right)^{5}, \quad \tau_{1}=\cdots=\tau_{5}=0,33, \quad c_{1}=\cdots=c_{5}=10, \quad R=I$, e $v_{\underline{\gamma}} \equiv 0$, foi gerada e tabulada uma amostra de $m=5.000$ observações da seqüência de Gibbs (4.13). A Tabela 4.1 exibe os quatro modelos correspondentes aos valores de $\gamma$ mais freqüentes, para ambos os casos.

Para o caso a), os dois modelos com as mais altas freqüências foram $\hat{Y}=f\left(Z_{5}\right)$ e $\hat{Y}=f\left(Z_{4}, Z_{5}\right)$. Apesar de $\beta_{4}=1$, portanto não nulo, a variável $Z_{4}$ foi freqüentemente excluída. Verifica-se que o valor da estatística $t$ para testar a hipótese $\beta_{4}=0$ é $\frac{\hat{\beta}_{4}}{\hat{\sigma}_{\beta_{4}}}=\frac{0,84}{0,31} \approx 2,7$. Já a estatística $t$ associada a $\beta_{5}$ foi aproximadamente 3,9 , proporcionando assim a inclusão da variável preditora $Z_{5}$ nos modelos, com alta freqüência. O método identifica diversos modelos promissores, o que nos permite uma escolha final dentro dessa classe de modelos. Observamos ainda que o modelo correto foi detectado como um dos mais freqüentes.

No caso (b), a freqüência com que ocorre $Z_{5}$ é quase a mesma com que ocorre $Z_{3}$, para ambos modelos, com as variáveis isoladamente ou incluindo a variável preditora $Z_{4}$. 
Tabela 4.1: Modelos com alta frequiência na seqüência de Gibbs, para o Exemplo 1

\begin{tabular}{ccccc}
\hline \multicolumn{2}{c|}{ Caso (a) } & \multicolumn{2}{c}{ Caso (b) } \\
Variáveis no modelo & Proporção & Variáveis no modelo & Proporção \\
\hline 5 & 0,258 & 3 & 0,146 \\
45 & 0,242 & 5 & 0,123 \\
25 & 0,070 & 34 & 0,098 \\
245 & 0,055 & 45 & 0,086 \\
\hline
\end{tabular}

Devido à relação existente entre $Z_{3}$ e $Z_{5}$, a saída da Tabela 4.1 para o caso b) pode ser considerada similar à do caso a). Observa-se ainda que a presença de multicolinearidade pode diluir as freqüências observadas de modelos contendo variáveis correlacionadas.

O segundo exemplo, exibido pelos autores, trata de um conjunto de dados envolvendo muitas variáveis preditoras, que deverão ser avaliadas quanto à entrada no modelo. No caso, trabalhou-se com 60 variáveis preditoras, $Z_{1}, \ldots, Z_{60}$ e 120 observações. As variáveis foram obtidas como $Z_{k}=Z_{\sim}^{*}+\underset{\sim}{Z}$, sendo que $Z_{1}^{*}, \ldots, Z_{60}^{*}$ são variáveis aleatórias independentes e identicamente distribuídas com distribuição $N_{120}(0, I)$ independentemente de $\underset{\sim}{Z} \sim N_{120}(0, I)$. Isto induziu correlações entre pares de variáveis $Z_{k}, Z_{k}$. de aproximadamente 0,5. A variável dependente foi gerada segundo o modelo

$$
Y=\left[Z_{1}, \ldots, Z_{60}\right] \beta+\varepsilon
$$


sendo que $\varepsilon \sim N_{120}\left(0, \sigma^{2} I\right)$ com $\sigma=2$. Os coeficientes de regressão $\beta^{\prime}=\left(\beta_{1}, \ldots, \beta_{60}\right)$ foram fixados de modo que

$$
\beta_{k}= \begin{cases}0, & k=1,2, \ldots, 15 \\ 1, & k=16, \ldots, 30 \\ 2, & k=31, \ldots, 45 \\ 3, & k=46, \ldots, 60 .\end{cases}
$$

Aplicando o método IASV com priori uniforme $f(\gamma) \equiv\left(\frac{1}{2}\right)^{60}, R=I$ e $v_{\underline{\gamma}} \equiv 0$, foram analisadas quatro situações $\left(\hat{\sigma}_{\beta_{k}} / \tau_{k}, c_{k}\right)=(1,5),(1,10)(10,100),(10,500)$. Para cada uma delas, foi simulada e tabulada uma amostra de $m=30.000$ observações da sequiência de Gibbs (4.13). O artigo exibe os cinco modelos que tiveram os mais altos valores de frequiências de $\gamma$ para cada situação.

Para $\left(\hat{\sigma}_{\beta_{k}} / \tau_{k}, c_{k}\right)=(1,5)$ e $\left(\hat{\sigma}_{\beta_{k}} / \tau_{k}, c_{k}\right)=(10,100)$, a investigação funcionou muito bem. Em ambas as situações, a escolha do modelo foi adequada, ou seja, não houve nenhuma escolha errônea de coeficientes de regressão para o modelo com a maior frequiência. Dizemos que a escolha de uma variável foi errônea quando incluímos variáveis de $Z_{1}$ a $Z_{15}$ ou quando excluímos variáveis de $Z_{16}$ a $Z_{60}$. Nas quatro maiores frequiências seguintes, os modelos tinham somente uma ou duas variáveis incluídas ou excluídas incorretamente. Nas situações em que $\left(\hat{\sigma}_{\beta_{k}} / \tau_{k}, c_{k}\right)=(1,10)$ e $\left(\hat{\sigma}_{\beta_{k}} / \tau_{k}, c_{k}\right)=(10,500)$, a investigação não foi tão eficaz, já que houve mais escolhas incorretas, principalmente, excluindo erroneamente variáveis que possuem coeficientes não nulos. Tais coeficientes eram, no entanto, pequenos. Aparentemente, isto foi consequiência do aumento dos valores de $c_{k}$ 's na simulação, acarretando decréscimo nas probabilidades de inclusão no modelo. Os 
autores sugerem que a simulação seja feita variando os valores de $\tau_{k}$ e $c_{k}$ para identificar os modelos mais adequados.

Para ilustrar a aplicação de IASV em um problema real, os autores utilizaram os dados conhecidos de Hald (Draper e Smith, 1998), que têm sido considerados por diversos outros autores para ilustrar seleção de variáveis, e que se encontram na Seção 5.1. Os dados consistem de $n=13$ observações de uma variável resposta e quatro variáveis preditoras. Aplicando a priori uniforme $\underset{\sim}{f(\gamma)} \equiv\left(\frac{1}{2}\right)^{4}, R=I$, e $v_{\gamma} \equiv 0$, foram analisadas quatro situações $\left(\hat{\sigma}_{\beta_{k}} / \tau_{k}, c_{k}\right)=(1,5),(1,10)(10,100)$, (10,500), já discutidas anteriormente. Para cada situação, foi simulada e tabulada uma amostra de $m=5.000$ observações da seqüência de Gibbs. Exibimos na Tabela 4.2 as freqüências de ocorrência dos modelos para os dados de Hald, em cada situação. Foram listados somente os modelos que apresentaram freqüência mínima de 0,02 em pelo menos uma seqüência de $\gamma^{1}, \ldots, \gamma^{m}$, dentre as quatro situações consideradas. 
Tabela 4.2: Freqüências de ocorrência dos modelos na seqüência de Gibbs para os dados de Hald

\begin{tabular}{ccccc}
\hline \multirow{2}{*}{ Variáveis no modelo } & \multicolumn{3}{c}{$\left(\hat{\sigma}_{\beta_{k}} / \tau_{k}, c_{k}\right)$} & \\
& $\mathbf{( 1 , 5 )}$ & $\mathbf{( 1 , 1 0 )}$ & $\mathbf{( 1 0 , 1 0 0 )}$ & $\mathbf{( 1 0 , 5 0 0 )}$ \\
\hline Nenhuma & 0,23 & 0,44 & - & - \\
1 & 0,26 & 0,25 & - & - \\
2 & 0,06 & 0,05 & - & - \\
3 & 0,06 & 0,06 & - & - \\
4 & 0,08 & 0,07 & - & 0,81 \\
1,2 & 0,07 & 0,03 & 0,60 & - \\
1,3 & 0,06 & 0,03 & - & 0,16 \\
1,4 & 0,06 & 0,03 & 0,27 & 0,01 \\
$1,2,3$ & 0,02 & 0,00 & 0,03 & 0,01 \\
$1,2,4$ & 0,02 & 0,00 & 0,05 & 0,01 \\
$1,3,4$ & 0,01 & 0,00 & 0,05 & \\
\hline
\end{tabular}

Nas situações $(1,5)$ e $(1,10)$, a investigação coloca mais probabilidade em modelos com poucas ou nenhuma variável. Nas outras duas situações, $(10,100)$ e $(10,500)$, as maiores probabilidades estão nos modelos que foram escolhidos por métodos tradicionais como: Todas as Regressões de Subconjuntos, Eliminação de Backward, Seleção de Forward e Regressão Stepwise. Além disso, as distribuições a posteriori de $\gamma$ para estas últimas situações estão muito mais concentradas em um número menor de modelos. 


\subsection{Considerações Finais}

Existe uma extensa bibliografia sobre seleção de variáveis no contexto bayesiano, mas as abordagens que apresentamos são as básicas e principais.

Uma nova linha de análise consiste em incorporar a incerteza sobre a validade de cada possível modelo no processo de inferência que é feito subseqüentemente.

Autores adeptos dessa abordagem argumentam que, embora o método de seleção de variáveis seja reconhecidamente a base de uma boa inferência, paradoxalmente, esse processo não é integrado na inferência. Desse modo, a inferência obtida é condicional ao modelo selecionado e totalmente dependente da validade do mesmo.

Uma solução para tal problema seria ponderar modelos competidores, ao invés de selecionar um deles. Essa análise é viável num contexto bayesiano, que forneceria estimativas das probabilidades de cada possível modelo ser correto. Referências sobre essa metodologia são Buckland, Burnham e Augustin (1997), Berger e Pericchi (1996), Hoeting, Madigan, Raftery e Volinsky (1999) e Draper (1995). 


\section{Capítulo 5}

\section{ANÁLISE DO CONJUNTO DE DADOS DE HALD}

\subsection{Introdução}

O conjunto de dados de Hald tem sido sistematicamente utilizado nos livros clássicos de regressão, para ilustrar os métodos tradicionais de seleção de variáveis. O conjunto é formado por $n=13$ amostras de tijolos de cimento para as quais foram avaliadas as variáveis preditoras

$Z_{1}=$ quantidade de $3 \mathrm{CaO} \cdot \mathrm{Al}_{2} \mathrm{O}_{3}$

$Z_{2}=$ quantidade de $3 \mathrm{CaO} \cdot \mathrm{SiO}_{2}$

$\mathrm{Z}_{3}=$ quantidade de $4 \mathrm{CaO} \cdot \mathrm{Al}_{2} \mathrm{O}_{3} \cdot \mathrm{Fe}_{2} \mathrm{O}_{3}$

$Z_{4}=$ quantidade de $2 \mathrm{CaO} \cdot \mathrm{SiO}_{2}$

e a variável resposta 
$Y=$ calor envolvido na reação (em calorias por grama) numa amostra de tijolos de cimento.

Tabela 5.1: Dados de Hald

\begin{tabular}{rrrrr}
\hline$Z_{1}$ & $Z_{2}$ & $Z_{3}$ & $Z_{4}$ & $Y$ \\
\hline 7 & 26 & 6 & 60 & 78,5 \\
1 & 29 & 15 & 52 & 74,3 \\
11 & 56 & 8 & 20 & 104,3 \\
11 & 31 & 8 & 47 & 87,6 \\
7 & 52 & 6 & 33 & 95,9 \\
11 & 55 & 9 & 22 & 109,2 \\
3 & 71 & 17 & 6 & 102,7 \\
1 & 31 & 22 & 44 & 72,5 \\
2 & 54 & 18 & 22 & 93,1 \\
21 & 47 & 4 & 26 & 115,9 \\
1 & 40 & 23 & 34 & 83,8 \\
11 & 66 & 9 & 12 & 113,3 \\
10 & 68 & 8 & 12 & 109,4 \\
\hline
\end{tabular}

Fonte: Draper e Smith (1998)

Com base nesse conjunto de dados, Draper e Smith (1998) aplica e descreve quatro procedimentos tradicionais de seleção em modelos com intercepto e variáveis não padronizadas. Quando as variáveis estão padronizadas, o modelo com intercepto apresenta intercepto nulo. Neste caso, vale lembrar que, independentemente da padronização, selecionamos as mesmas variáveis preditoras. O modelo $\hat{Y}=f\left(Z_{1}, Z_{2}\right)$ com $R^{2}=97,9 \%$ e $S^{2}=Q M \operatorname{Re} s=5,79$ foi selecionado pelos métodos Todas as possíveis regressões, Backward e Stepwise; além disso, no método Todas as possíveis regressões, também destacou-se o modelo $\hat{Y}=\left(Z_{1}, Z_{4}\right)$, com $R^{2}=97,2 \%$ e $Q M \operatorname{Re} s=7,48$, sendo este menos utilizado devido ao alto valor do quadrado médio do resíduo. O modelo $\hat{Y}=\left(Z_{1}, Z_{2}, Z_{4}\right)$, que apresenta $R^{2}=98,2 \%$, foi obtido através do método Forward.

Neste capítulo, aplicaremos inicialmente o método de seleção de variáveis Bayesiano com o objetivo de selecionar variáveis preditoras no conjunto de dados da Tabela 5.1. Posteriormente, será selecionado o melhor modelo de regressão através 
do método Lasso. A análise computacional relativa ao método Bayesiano foi desenvolvida no pacote S-Plus (2000) e as está descrita no Apêndice B. Já, o método Lasso foi desenvolvido no programa Excell.

\subsection{Método Bayesiano}

De acordo com a teoria apresentada na Seção 4.2, o cálculo básico para cada um dos $2^{4}=16$ possíveis modelos é

$$
\operatorname{Pr}\left(A_{m} / y\right)=g^{\prime} \gamma^{-q_{m}} \pi^{q_{m} / 2} \Gamma\left(\frac{n-q_{m}}{2}\right) \cdot\left|Z_{m}{ }^{\prime} Z_{m}\right|^{-1 / 2}\left(S_{m}^{2}\right)^{-\left[\left(n-q_{m}\right) / 2\right]}
$$

em que $A_{m}$ representa o $m$ - ésimo modelo, $m=1,2, \ldots, 16$.

O valor de $\operatorname{Pr}\left(A_{m} / y\right)$ foi obtido para diferentes valores de $\gamma$ conforme descrito em B.1 do Apêndice B. Foram utilizados $\gamma=1,23 \times 10^{3}, 5 \times 10^{8}, 11 \times 10^{12}$, $25 \times 10^{16}, 55 \times 10^{20}$, cujo logaritmo na base $e$ resulta nos valores $0,10,20,30,40,50$. A constante normalizadora $g$ foi determinada a partir de $\operatorname{Pr}\left(A_{m} / y\right)$, de modo que $\sum_{m=1}^{16} \operatorname{Pr}\left(A_{m} / y\right)=1$ e a descrição do cálculo encontra-se em B.2.

A probabilidade a posteriori dada por

$$
\operatorname{Pr}\left(\beta_{k}=0 / y\right)=\sum \operatorname{Pr}\left(A_{m} / \underline{y}\right)
$$

onde a soma é calculada sobre todos submodelos $A_{m}$ que não incluem o coeficiente $\beta_{k}$, é usada para avaliar a importância de um determinado coeficiente de regressão. A parte computacional está descrito em B.3. 
O número esperado de termos em cada modelo,

$$
E\left(q_{m} / \underset{\sim}{y}\right)=\sum_{m=1}^{2 q} q_{m} \operatorname{Pr}\left(A_{m} / \underset{\sim}{y}\right),
$$

foi determinado pelo procedimento presente em B.4.

No tópico B.5 está o cálculo da perturbação a posteriori da distribuição do submodelo $A_{m}$, definida por

$$
H=-\sum_{m=1}^{24} \operatorname{Pr}\left(A_{m} / \underset{\sim}{y}\right) \ln \left(\operatorname{Pr}\left(A_{m} / \underset{\sim}{y}\right)\right) .
$$

Para cada valor de $\gamma$, o erro preditivo é obtido através da expressão

$$
E P=\sqrt{\frac{1}{n} \sum_{i=1}^{n} E Q M_{i}},
$$

sendo que $E Q M_{i}$ é o erro quadrático médio do modelo ajustado sem a $i$-ésima observação (descrito em B.6). A quantidade $E P$ foi obtida na expressão dada em B.7.

Foram construídos gráficos das seguintes quantidades em função de $\ln \gamma$ :

(a) Probabilidade a posteriori de $\beta_{k}=0, \operatorname{Pr}\left(\beta_{k}=0 / y\right)$;

(b) Número esperado de termos (a posteriori) no submodelo, $E\left(q_{m} / y\right)$;

(c) Antilogaritmo da perturbação (a posteriori) da distribuição do submodelo, $\exp (H) ;$

(d) Erro preditivo a posteriori, $E P$; 
Tais gráficos estão apresentados na Figura 5.1.

Figura 5.1: Gráficos associados ao método de seleção Bayesiano para o conjunto de dados de Hald.

(a) $\operatorname{Pr}\left(\beta_{k}=0 / y\right)$

(b) $E\left(q_{m} / y\right)$
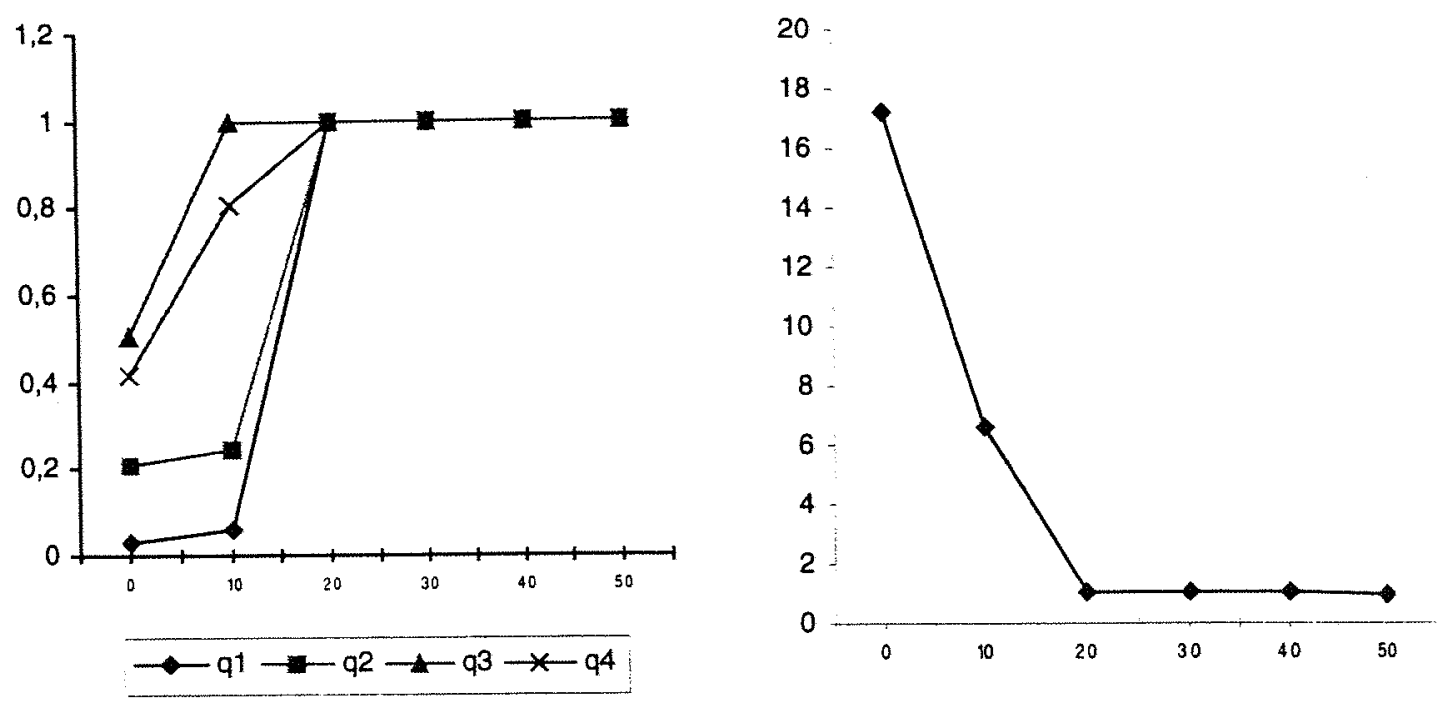

(c) $\exp (H)$

(d) $E P$
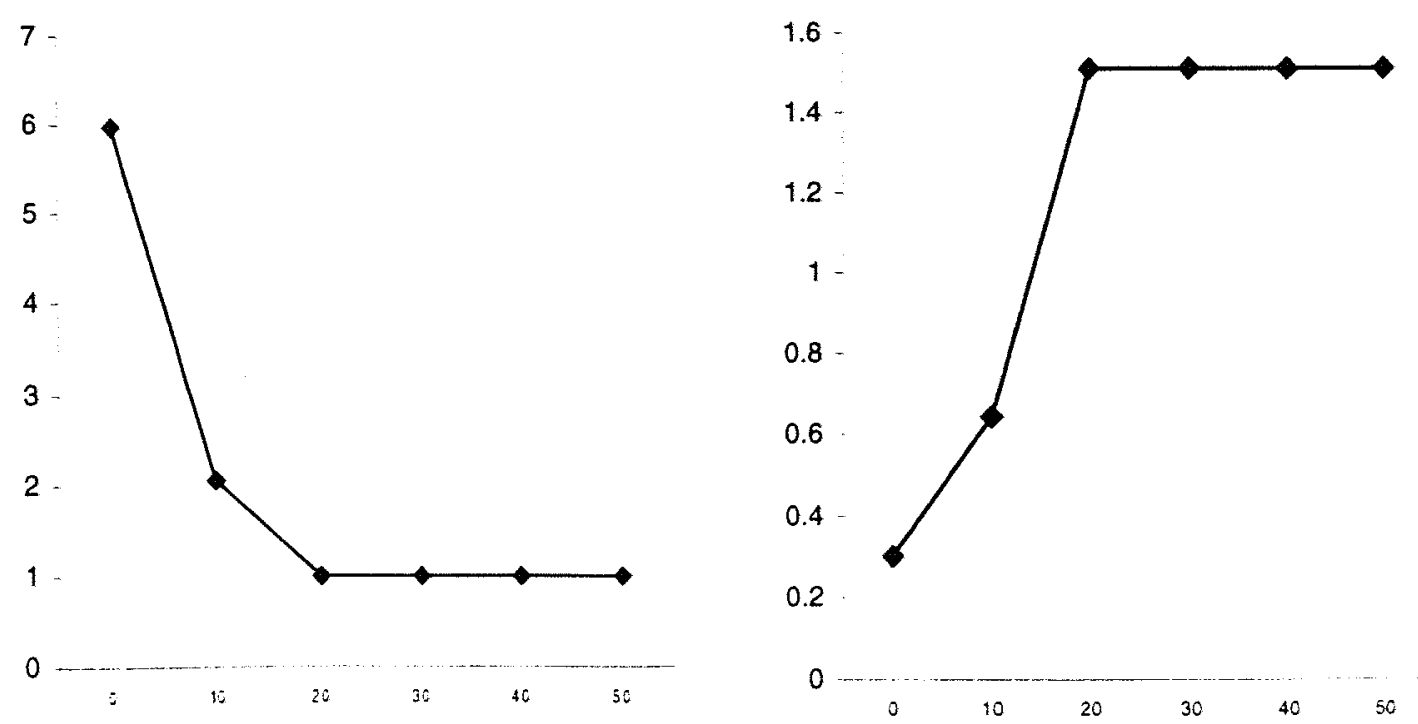
Devido à inexistência de réplicas nas variáveis preditoras para alguns modelos, não foi possível executar o teste de falta de ajuste, impossibilitando a construção do gráfico da probabilidade a posteriori da qualidade do ajuste, $Q$.

Através do critério descrito na Seção 4.2 escolhemos o valor $\gamma=10$ pois fornece baixos valores de $E\left(q_{m} / y\right), \exp (H)$ e $E P$. Para esse valor de $\gamma$, através do gráfico (a) da Figura 5.1 observamos que as variáveis preditoras selecionadas serão as mesmas que o método Forward, $Z_{1}, Z_{2}$ e $Z_{4}$.

Uma outra possível seleção seria a das variáveis $Z_{1}$ e $Z_{2}$ já que, conforme pode-se notar neste gráfico, $P\left(\beta_{4}=0 / y\right)=0,8$. Neste caso, o modelo final coincidiria com o modelo selecionado pelos métodos Backward, Stepwise e Todas as possíveis regressões.

\subsection{Lasso}

No ajuste pelo método Lasso, utilizou-se a ferramenta solver do Excell na obtenção das estimativas dos coeficientes do modelo através de minimização da soma de quadrado do resíduo sujeito à restrição $\sum_{k=1}^{q} \mid \beta_{k} \leqslant s$. Apenas algumas versões do Excell possuem este aplicativo, geralmente as versões destinadas às universidades.

Para se obter as estimativas dos coeficientes no conjunto de dados de Hald, são alocadas em quatro células as estimativas iniciais dos coeficientes, com valores nulos. Reservamos o mesmo número de células para os valores absolutos dessas quantidades e uma outra célula com a soma de tais valores. A célula contendo a soma não poderá ultrapassar o valor $s^{*}=s / \sum_{k=1}^{q}\left|\beta_{k}^{0}\right|$, sendo que $\beta_{k}^{0}$ corresponde às 
estimativas de mínimos quadrados. Destinamos uma célula para inserir o valor de $s^{*}$, que será alterado a cada repetição do processo. O valor estimado para $s, \hat{s}^{*}$, será o do modelo que tiver a menor estimativa do erro de previsão.

A estimativa do erro de previsão para cada valor de $s$ foi obtida através do método Cross-Validation, com 5 partições, conforme descrito na Seção 3.2, e é dada por

$$
\hat{E P}(s)=\frac{1}{5} \sum_{v=1}^{5} \hat{E P}^{v}(s), \text { para } s=1,2,3,4,
$$

sendo que $v=1,2, \ldots, 5$ corresponde ao índice do conjunto teste na $v$-ésima partição.

Com esse objetivo, as 13 observações foram partidas nos conjuntos $\{1,2,3\}$, $\{4,5,6\},\{7,8,9\},\{10,11\},\{12,13\}$. Dessa forma, para $v=1$, o conjunto teste era $T^{1}=\{1,2,3\}$ e o modelo era ajustado com base nas observações restantes $T-T^{1}=\{4,5, \ldots, 13\}$, de acordo o método Lasso com valor de $s$ fixado. Obtinha-se então $\sum_{i=1}^{3}\left(y_{i}-\hat{y}_{i T-T^{1}}\right)^{2}$, sendo que $\hat{y}_{i T-T^{1}}$ é o previsor de $y_{i}$ sob o modelo ajustado. Procedeu-se assim, sucessivamente, para $v=2,3,4,5$, obtendo-se $\hat{E P}(s)$ para cada $s$. O valor de $s$ que minimizou $\hat{E P}(s)$, foi o selecionado. Computacionalmente, o cálculo de

$$
\sum_{j \in T^{v}}\left(y-\hat{y}_{T-T^{v}}\right)^{2}
$$

foi feito da seguinte maneira:

Para cada $s$ e partição, devemos calcular $\hat{Y}=Z_{1} B_{1}+Z_{2} B_{2}+Z_{3} B_{3}+Z_{4} B_{4}$, e em seguida, $(Y-\hat{Y})^{2}$, associado a cada observação. Ao somarmos os valores obtidos em 
$(Y-\hat{Y})^{2}$ para todas observações obtemos o resultado para a equação 5.1. Para realização do procedimento, ou seja, na obtenção de $\hat{Y}$, aplicamos o comando Ferramentas $>$ Solver, abrindo-se em seguida a tela que pode ser visualizada em C.1 do Apêndice $\mathrm{C}$, com os dados conforme expostos. Definimos as células de destino para soma de quadrado do resíduo, selecionamos o comando min para minimizá-la. As células variáveis serão os coeficientes de regressão, e a restrição a qual foi submetido o procedimento será tal que soma dos coeficientes absolutos seja menor que o valor de $s^{*}$.

Ao resolver, obtemos outra tela que poderá ser visualizada em C. 2 do apêndice $\mathrm{C}$, juntamente com a resolução obtida pelo método. Podemos optar em manter a solução do solver ou restaurar os valores originais para uma nova adequação.

Usando o valor de $s$ selecionado, calculamos através da aplicação do solver as estimativas dos coeficientes de regressão e, conseqüentemente, as variáveis que farão parte do modelo.

Aplicado o procedimento descrito ao conjunto de dados de Hald, o valor selecionado de $\hat{s}^{*}$ foi $\hat{s}^{*}=0,87$. O modelo ajustado foi $\hat{y}=0,37 z_{1}+0,27 z_{2}-0,23 z_{4}$, de modo que o método Lasso selecionou as mesmas variáveis que o método Forward. O modelo ajustado por mínimos quadrados contendo as mesmas variáveis preditoras padronizadas é $\hat{y}=0,57 z_{1}+0,43 z_{2}-0,26 z_{4}$.

Neste conjunto de dados, observou-se então uma forte coerência entre os métodos Bayesiano e Lasso e os métodos tradicionais. Destacamos que o procedimento Lasso eliminou a variável $Z_{3}$, assim como todos os demais métodos. Com relação ao método Bayesiano, embora seja teoricamente atraente, é de difícil implementação. 


\section{APÊNDICE A - ESTIMADOR LASSO}

Considere $\beta$ um vetor num espaço paramétrico $q$-dimensional, $Z$ uma matriz de planejamento $(n \times q)$, e y um vetor pertencente ao espaço amostral $n$ dimensional, $R^{n}$. Para $Z$ e $y$ fixados, vamos supor que $q=2$, ou seja, o caso envolve apenas dois parâmetros $\beta_{1}$ e $\beta_{2}$, e que $n=3$, de modo que $y \in R^{3}$. Apresentaremos a seguir uma justificativa geométrica para a expressão do estimador Lasso dada na Seção 3.3.

Sejam $Z^{1}=\left(z_{11}, z_{12}, z_{13}\right)$ e $Z^{2}=\left(z_{21}, z_{22}, z_{23}\right)$ vetores correspondendo à primeira e à segunda coluna da matriz de planejamento $Z$, respectivamente. Pode se dizer que $\operatorname{sinal}\left(\hat{\beta}_{k}\right), \quad k=1,2$, identifica o quadrante que está o ponto $\hat{\beta}=\hat{\beta}_{1} Z^{1}+\hat{\beta}_{2} Z^{2}$ no sistema cartesiano $\hat{\beta}_{1}$ e $\hat{\beta}_{2}$.

Denotaremos por $\hat{\beta}^{0}$ o estimador de mínimos quadrados que é obtido pela projeção perpendicular do ponto $y$ no plano $\pi_{0}$ gerado por $Z^{1}$ e $Z^{2}$. As estimativas dos parâmetros através do procedimento Lasso, são definidas a partir da minimização da quantidade

$$
\left\|y-\left(\beta_{1} Z^{1}+\beta_{2} Z^{2}\right)\right\|_{2}^{2}=\sum_{i=1}^{n}\left(y_{i}-\sum_{k=1}^{q} \beta_{k} z_{k i}\right)^{2}
$$

sujeita à condição $\sum_{k=1}^{q}\left|\beta_{k}\right| \leq s$.

O problema se resume em encontrar o ponto pertencente ao paralelogramo $Q=\left\{\beta_{1} Z^{1}+\beta_{2} Z^{2} /\left|\beta_{1}\right|+\left|\beta_{2}\right| \leq s\right\}$, que está mais próximo do ponto $\hat{\beta}^{0}$. Quanto maior for o valor do parâmetro $s$, maior será a possibilidade da projeção do ponto $\hat{\beta}^{0}$ 
encontrar-se dentro do conjunto $Q$. No caso do método Lasso, para exemplificar, estaremos admitindo que $\left\{Z^{1}, Z^{2}\right\}$ é um conjunto ortonormal e que $s=1$, e assim a figura gerada será um quadrado com os vértices nos eixos $\hat{\beta}_{1}$ e $\hat{\beta}_{2}$. Se o ponto $\hat{\beta}^{0}=\hat{\beta}_{1}^{0} Z^{1}+\hat{\beta}_{2}^{0} Z^{2}$ estiver fora do quadrado $Q$, na busca da solução devemos encontrar o ponto $\hat{\beta}=\hat{\beta}_{1} Z^{1}+\hat{\beta}_{2} Z^{2} \in Q$ mais próximo de $y$, que normalmente fica na fronteira do quadrado $Q$. Se o ponto $\hat{\beta}^{0}=\hat{\beta}_{1}^{0} Z^{1}+\hat{\beta}_{2}^{0} Z^{2}$ estiver dentro do quadrado $Q$ então temos que $\hat{\beta}=\hat{\beta}^{0}$.

Suponhamos sem perda de generalidade que as estimativas $\hat{\beta}_{k}^{0}, k=1,2$, são ambas positivas.

Com objetivo de encontrar o ponto $\hat{\beta}=\hat{\beta}_{1} Z^{1}+\hat{\beta}_{2} Z^{2} \in Q$ mais próximo de $y$, projetamos o ponto $\hat{\beta}^{0}$ perpendicularmente à reta $r$, que está sobreposta no lado do quadrado do primeiro quadrante do sistema cartesiano $\hat{\beta}_{1}$ por $\hat{\beta}_{2}$, ver Figura A1. A reta para a projeção será sempre aquela que estiver no mesmo quadrante do ponto $\hat{\beta}^{0}$, quadrante este determinado pelos sinais das componentes $\hat{\beta}_{1}^{0}$ e $\hat{\beta}_{2}^{0}$.

As Figuras A1 e A2 exibem $Z^{1} \perp Z^{2}$, gerando um plano $\pi_{0}$ que passa pela origem, e $Q$ é um quadrado contido em $\pi_{0}$. Quando se projeta o ponto $\hat{\beta}^{0}$ na reta conveniente $r$, pode ocorrer que se obtenha um ponto de $Q$, ou um ponto fora de $Q$.

No primeiro caso, o ponto de $Q$ obtido é o próprio $\hat{\beta}$ procurado.

No segundo caso, o segmento que une $\hat{\beta}^{0}$ à sua projeção na reta $r$ cruza algum dos eixos. Neste caso devemos anular a estimativa do coeficiente de regressão correspondente ao eixo restante. Em seguida, deve-se percorrer sobre este eixo em direção à origem até cruzarmos a fronteira da figura $Q$, obtendo $\hat{\beta}$. Dessa forma, obtém-se a estimativa do coeficiente correspondente ao eixo que percorremos. 
Figura A1: Primeiros procedimentos para obtenção da estimativa Lasso $\hat{\beta}$

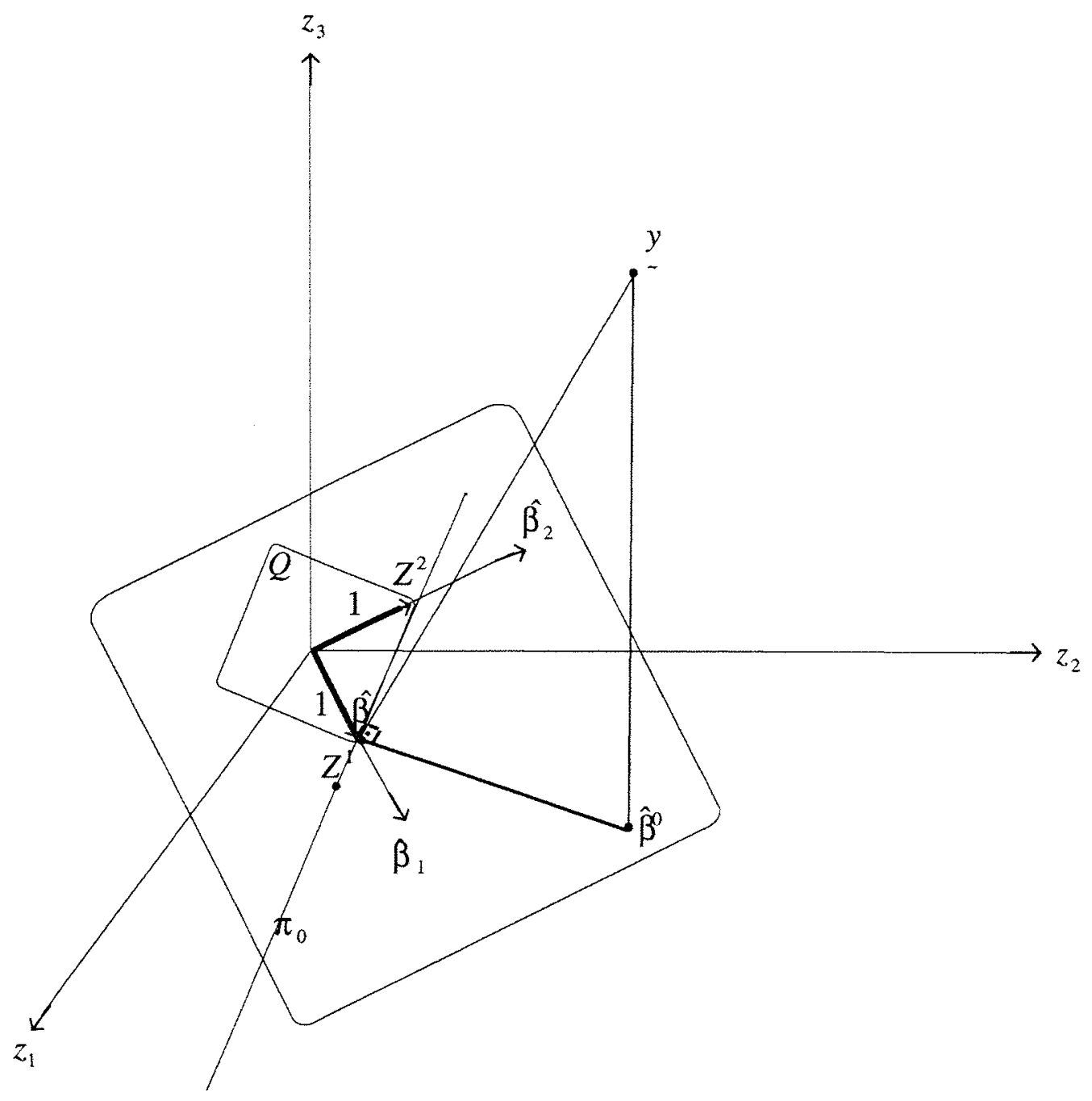


Figura A2: Obtenção da estimativa Lasso $\hat{\beta}$

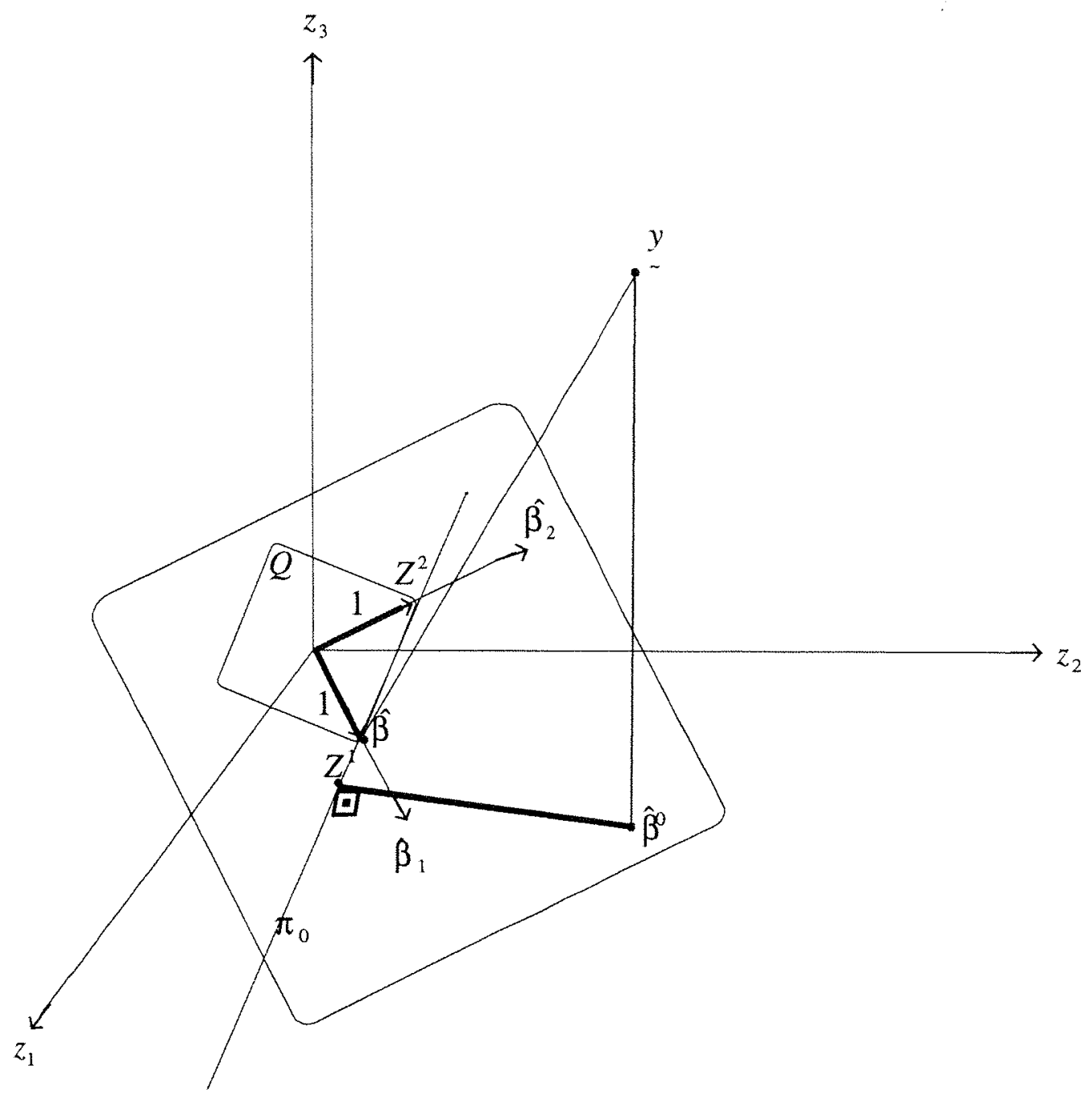




\section{APÊNDICE B - ASPECTOS COMPUTACIONAIS}

Apresentaremos a seguir as principais etapas e comandos para a seleção de variáveis através do método Bayesiano, usando o aplicativo S-Plus (2000).

Foram construídos matrizes para alocar os valores das variáveis preditoras e da variável resposta. Os modelos são representados por $\mathrm{Am}$, onde $m$ corresponde ao índice do modelo, e a variável resposta pode ser identificada no programa por $Y t$. Para cada modelo foi definida a variável $q A m$ cujo valor é o número de variáveis preditoras no modelo $m$.

Construímos outras matrizes da mesma forma descrita acima, mas agora retirando cada observação dos dados. Sendo assim, A1s1, representa o modelo 1 definido por nós, sem a primeira observação. Construímos todas as matrizes possíveis, para todos os modelos sem a primeira observação, em seguida para todos os modelos sem a segunda observação, e assim por diante.

O próximo passo foi ajustar o modelo sem intercepto por mínimos quadrados usando o comando

$$
\begin{aligned}
& \text { fitOLSA1<-coef }\left(\operatorname { l m } \left(\text { Yt } \sim-1+A 1, \text { method=" } q r^{\prime}, \text { model=F, } x=F, y=F,\right.\right. \\
& \text { contrasts=NULL })) .
\end{aligned}
$$

Acionamos o coeficiente do modelo 1 através de

$$
\text { BestA1[1,]<-coef(fitOLSA1)[1]. }
$$

Indicamos que a matriz BestAls 1 contém o coeficiente do modelo 1 sem a primeira observação, seguindo a mesma lógica para os demais modelos.

$O$ determinante de $A l^{\prime} A 1$ é representado por

$$
\operatorname{dettA1A1<-det.Matrix(t(A1)\% *\% A1,logarithm=F),~}
$$


porém é acionado por

$$
\text { vdettA1A1<-dettA1A1\$modulus[1]*dettA1A1\$sign. }
$$

Além disso, é necessária a soma de quadrados de resíduo que poderá ser conseguida pela forma tradicional, por exemplo, para o modelo 1 sem a primeira observação, por

$$
\text { (S2A1s1<-t(Ys1-A1s1\%*\%BestA1s1)\%*\%(Ys1-A1s1\%*\%BestA1s1)) }
$$

ou por

$$
(\mathrm{S} 2 \mathrm{~A} 1 \mathrm{~s} 1<-\mathrm{S} 2 \mathrm{~A} 1-\mathrm{eA} 1[1] * \mathrm{f} 1 \mathrm{~A} 1)
$$

sendo que o vetor de resíduos de mínimos quadrados, eA1<-Yt-A1\%*\%BestA1, conterá eA1[1], e f1A1<-eA1[1]/(1-hA1[1]) é obtido utilizando, além do resíduo, a diagonal da matriz determinada por $\mathrm{hA} 1<-\operatorname{diag}\left(\mathrm{A} 1 \% * \%(\mathrm{t}(\mathrm{A} 1) \% * \% \mathrm{~A} 1)^{\wedge}(-\right.$ $1) \% * \% t(\mathrm{~A} 1))$.

B.1 Para o modelo $A 1$, o cálculo de $\operatorname{Pr}\left(A_{1} / y\right)$ é feito pela fórmula

$\operatorname{PrA} 1<-$

$\left(\operatorname{gama}{ }^{\wedge}(-\mathrm{qA} 1)\right)^{*} \mathrm{pi}^{\wedge}(\mathrm{qA} 1 / 2)^{*} \operatorname{gamma}(((\mathrm{n}-1) / 2))^{*} \operatorname{sqrt}(1 / \mathrm{vdettA} 1 \mathrm{~A} 1)^{*}(\mathrm{~S} 2 \mathrm{~A} 1)^{\wedge}(-(\mathrm{n}-$ $\mathrm{qA} 1) / 2)$.

B.2 Cálculo da constante normalizadora

G<-sum(PrA1,PrA2,PrA3,PrA4,PrA5,PrA6,PrA7,PrA8,PrA9,PrA10,PrA11,PrA12, PrA13,PrA14,PrA15,PrA16).

Para cada modelo redefinimos PrA1 de acordo com a constante normalizadora da seguinte forma 
$\operatorname{PrA} 1<-(1 / \mathrm{G}) * \operatorname{PrA} 1$.

B.3 Probabilidade a posteriori

PrBq0q1<-sum(PrA2,PrA3,PrA4,PrA8,PrA9,PrA10,PrA14,PrA16)

PrBq0q2<-sum(PrA1,PrA3,PrA4,PrA6,PrA7,PrA10,PrA13,PrA16)

PrBq0q3<-sum(PrA1,PrA2,PrA4,PrA5,PrA7,PrA9,PrA12,PrA16)

PrBq0q4<-sum(PrA1,PrA2,PrA3,PrA5,PrA6,PrA8,PrA11,PrA16),

B.4 Número esperado de termos

Espqm<-sum(qA1*PrA1,qA2*PrA2,qA3*PrA3,qA4*PrA4,qA5*PrA5,qA6*PrA6, qA7*PrA7,qA8*PrA8,qA9*PrA9,qA10*PrA10,qA11*PrA11,qA12*PrA12, qA13*PrA13,qA14*PrA14,qA15*PrA15,qA16*PrA16).

B.5 Perturbação a posteriori da distribuição do modelo

$\mathrm{H}<--\operatorname{sum}(\operatorname{PrA} 1 * \log (\operatorname{PrA} 1), \operatorname{Pr} \mathrm{A} 2 * \log (\operatorname{Pr} \mathrm{A} 2), \operatorname{PrA} 3 * \log (\operatorname{Pr} \mathrm{A} 3), \operatorname{Pr} \mathrm{A} 4 * \log (\operatorname{Pr} \mathrm{A} 4)$, PrA5* $\log ($ PrA5),PrA6* $\log (\operatorname{PrA6}), \operatorname{PrA} 7 * \log (\operatorname{PrA} 7), \operatorname{PrA} 8 * \log (\mathrm{PrA} 8)$, PrA9* $\log (\operatorname{Pr} A 9), \operatorname{Pr} A 10 * \log (\operatorname{Pr} A 10), \operatorname{Pr} A 11 * \log (\operatorname{PrA} 11), \operatorname{Pr} A 12 * \log (\operatorname{Pr} A 12)$, PrA13* $\log (\operatorname{PrA13}), \operatorname{PrA14} * \log (\operatorname{PrA14}), \operatorname{PrA15} * \log (\operatorname{PrA15}), \operatorname{PrA16*} \log (\operatorname{PrA16}))$,

B.6 Erro quadrático médio

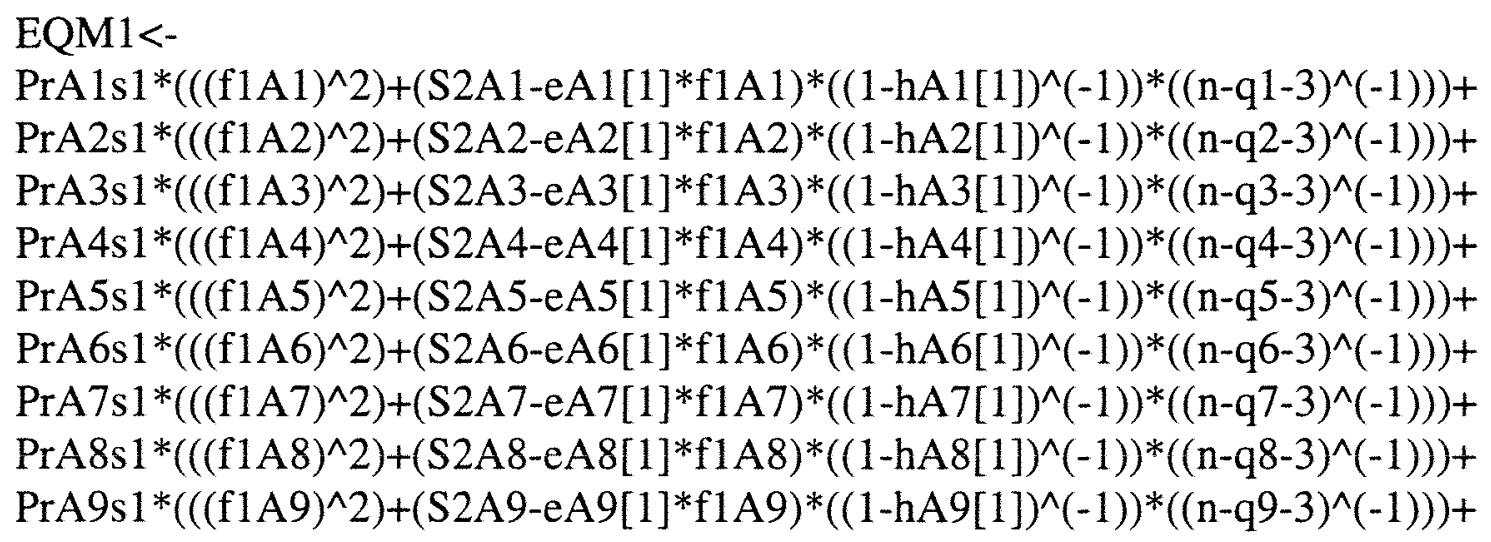


$\operatorname{PrA10s} 1 *\left(\left((\mathrm{f} 1 \mathrm{~A} 10)^{\wedge} 2\right)+\left(\mathrm{S} 2 \mathrm{~A} 10-\mathrm{eA} 10[1]^{*} \mathrm{f} 1 \mathrm{~A} 10\right) *\left((1-\mathrm{hA} 10[1])^{\wedge}(-1)\right)^{*}\left((\mathrm{n}-\mathrm{q} 10-3)^{\wedge}(-\right.\right.$ 1)))+

$\operatorname{PrA} 11 \mathrm{~s} 1 *\left(\left((\mathrm{f} 1 \mathrm{~A} 11)^{\wedge} 2\right)+\left(\mathrm{S} 2 \mathrm{~A} 11-\mathrm{eA} 11[1]^{*} \mathrm{f} 1 \mathrm{~A} 11\right) *\left((1-\mathrm{hA} 11[1])^{\wedge}(-1)\right)^{*}\left((\mathrm{n}-\mathrm{q} 11-3)^{\wedge}(-\right.\right.$ 1)))+

$\operatorname{PrA} 12 \mathrm{~s} 1 *\left(\left((\mathrm{f} 1 \mathrm{~A} 12)^{\wedge} 2\right)+\left(\mathrm{S} 2 \mathrm{~A} 12-\mathrm{eA} 12[1]^{*} \mathrm{f} 1 \mathrm{~A} 12\right) *\left((1-\mathrm{hA} 12[1])^{\wedge}(-1)\right)^{*}\left((\mathrm{n}-\mathrm{q} 12-3)^{\wedge}(-\right.\right.$ 1)))+ $\operatorname{PrA13s} 1 *\left(\left((\mathrm{f} 1 \mathrm{~A} 13)^{\wedge} 2\right)+\left(\mathrm{S} 2 \mathrm{~A} 13-\mathrm{eA} 13[1]^{*} \mathrm{f} 1 \mathrm{~A} 13\right) *\left((1-\mathrm{hA} 13[1])^{\wedge}(-1)\right) *\left((\mathrm{n}-\mathrm{q} 13-3)^{\wedge}(-\right.\right.$ 1)))+

$\operatorname{PrA14s} 1 *\left((\mathrm{f} 1 \mathrm{~A} 14)^{\wedge} 2\right)+\left(\mathrm{S} 2 \mathrm{~A} 14-\mathrm{eA} 14[1]^{*} \mathrm{f} 1 \mathrm{~A} 14\right) *\left((1-\mathrm{hA} 14[1])^{\wedge}(-1)\right)^{*}\left((\mathrm{n}-\mathrm{q} 14-3)^{\wedge}(-\right.$ 1)))+

$\operatorname{PrA15s} 1 *\left(\left((\mathrm{f} 1 \mathrm{~A} 15)^{\wedge} 2\right)+(\mathrm{S} 2 \mathrm{~A} 15-\mathrm{eA} 15[1] * \mathrm{f} 1 \mathrm{~A} 15)^{*}\left((1-\mathrm{hA} 15[1])^{\wedge}(-1)\right)^{*}\left((\mathrm{n}-\mathrm{q} 15-3)^{\wedge}(-\right.\right.$ 1)))+

$\operatorname{PrA16s} 1 *\left(\left((\mathrm{f} 1 \mathrm{~A} 16)^{\wedge} 2\right)+\left(\mathrm{S} 2 \mathrm{~A} 16-\mathrm{eA} 16[1]^{*} \mathrm{f} 1 \mathrm{~A} 16\right)^{*}\left((1-\mathrm{hA} 16[1])^{\wedge}(-1)\right)^{*}\left((\mathrm{n}-\mathrm{q} 16-3)^{\wedge}(-\right.\right.$ 1))),

após fazer o mesmo cálculo eliminando cada observação então utilizamos seus valores no erro preditivo

B.7 Erro preditivo

EP<-sqrt((1/n)*sum(QMRes1,QMRes2,QMRes3,QMRes4,QMRes5,QMRes6, QMRes7,QMRes8,QMRes9,QMRes10,QMRes11,QMRes12,QMRes13)). 


\section{APÊNDICE C - PROGRAMA PARCIAL DO LASSO}

C. 1

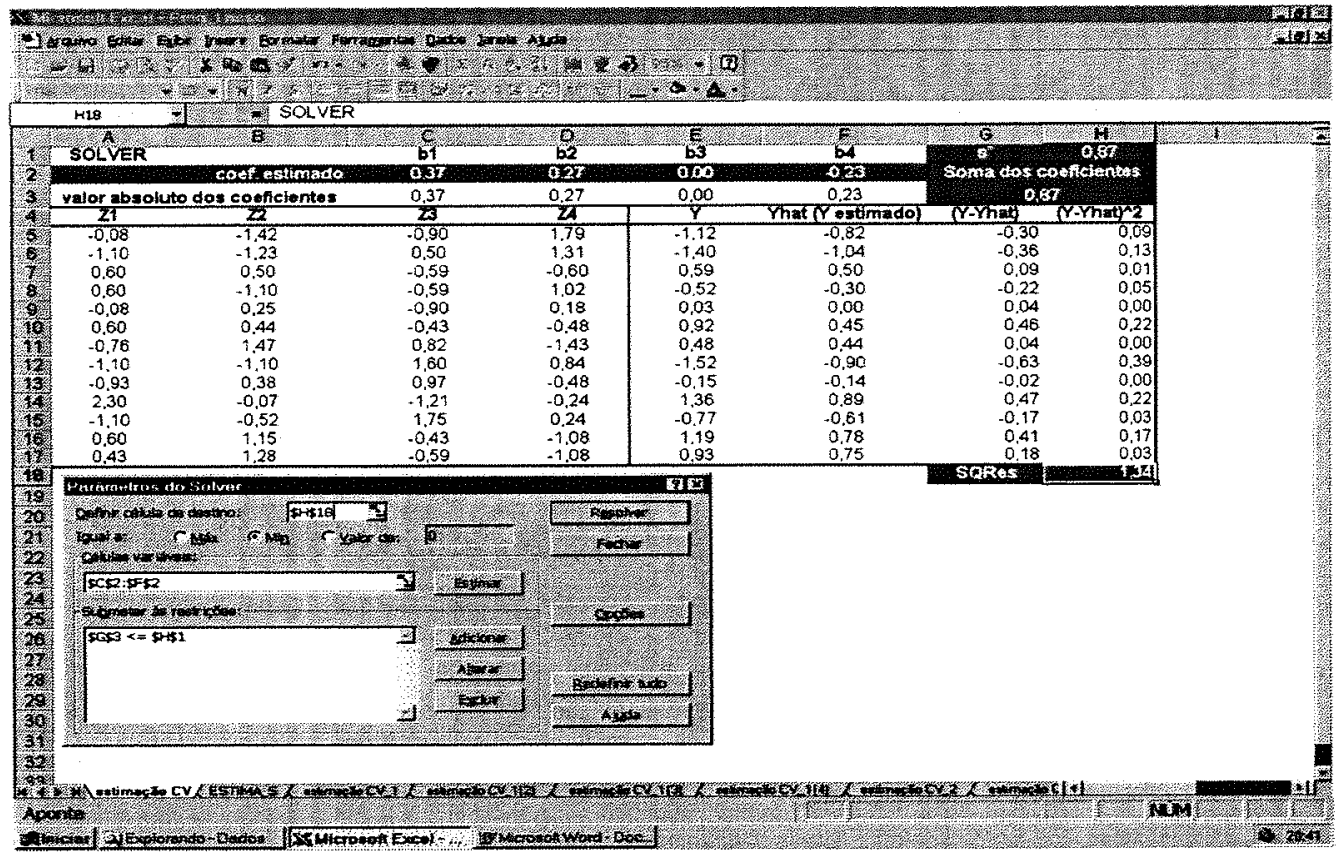

C. 2

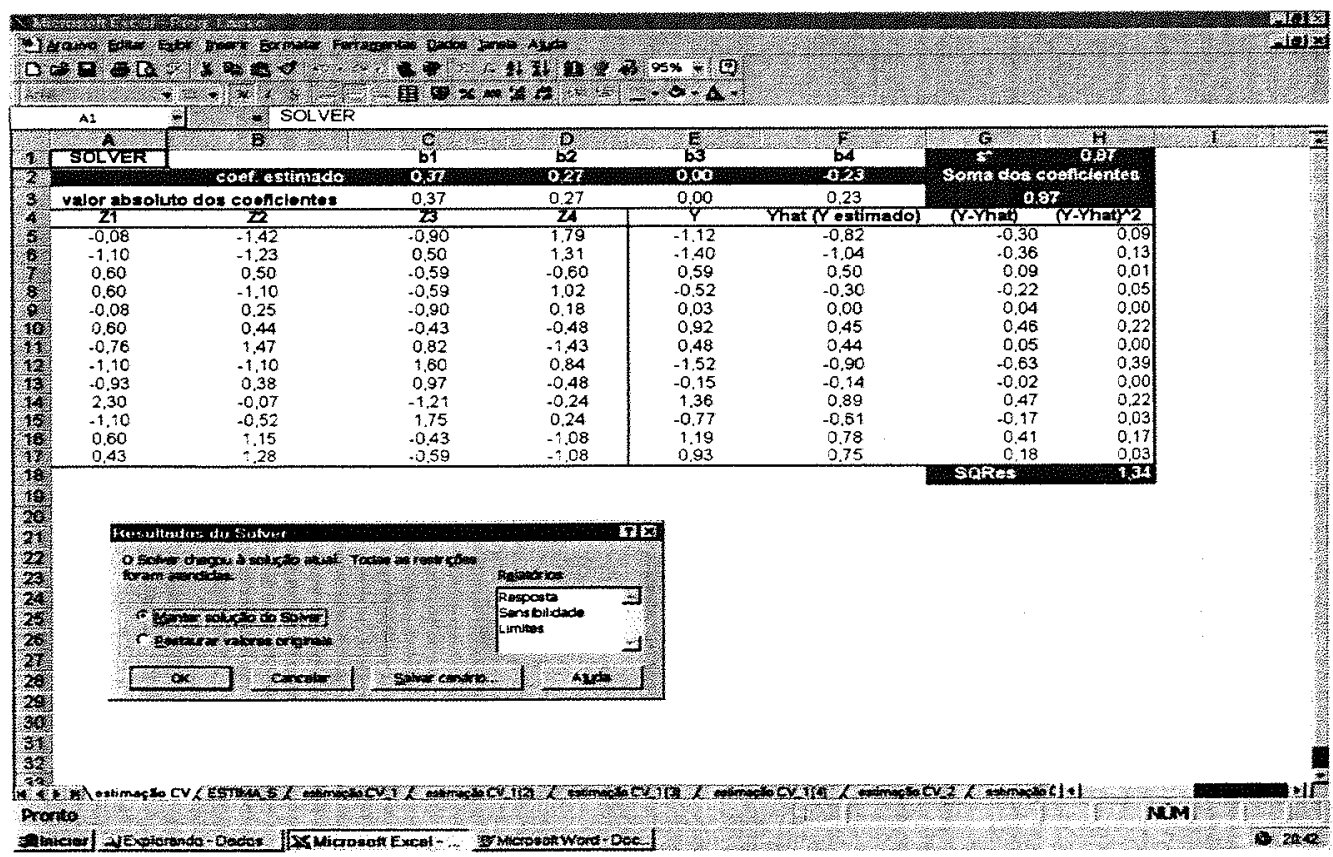




\section{REFERÊNCIAS BIBLIOGRÁFICAS}

Akaike, H. (1974). "A New Look at the Statistical Model Identification", IEEE Transations on Automatic Control, AC-19, $\mathrm{n}^{\circ}$ 6, 716-723.

Berger, J. O. and Pericchi, L. R. (1996). "The intrinsic Bayes factor for model selection and Prediction", Journal of the American Statistical Association 91, 109122.

Bernardo, J. M., Smith, A. F. M. (1994). Bayesian Theory, Wiley series in probability and mathematical statistics. 586p.

Breiman, L. (1992). "The Little Bootstrap and Other Methods for Dimensionality Selection in Regression: X-Fixed Prediction Error", Journal of the American Statistical Association, 87, 738-754.

Breiman, L. (1995). "Better Subset Selection Using the Nonnegative Garrote", Technometrics, 37, 373-384.

Breiman, L. (1996a). "The Heuristics of Instability in Model Selection", The Annals of Statistics, 24 (6), 2350-2383. 
Breiman, L. (1996b). "Stacked Regressions”, Machine Learning, 24, 49-64.

Buckland, S. T., Burnham K. P. and Augustin, N. H. (1997). "Modelo Selection: An Integral Part of Inference", Biometrics, 53, 603-618.

Draper, D. (1995). “Assessment and propagation of model uncertainty (with discussion)", Journal the Royal Statistical Society, Series B 57, 45-97.

Draper, N. R. and Smith, H. (1998). Applied Regression Analysis, Third Edition. New York: John Wiley \& Sons. 706p.

Furnival, G. and Wilson, R. (1974). "Regression by Leaps and Bounds", Technometrics, 16, 499-511.

Geisser, S. (1965). "Bayesian Estimation in Multivariate Analysis", Annals of Mathematical Statistics, 36: 150-159.

Geisser, S. (1975). "The Predictive Sample Reuse Method with Applications", Journal of the American Statistical Association, 70: 320-328.

Geisser, S. and Eddy, W. F. (1979). "A Predictive Approach to Model Selection", Journal of the American Statistical Association, 74 (365): 150-153.

Geisser, S. and Eddy, W. F. (1980). "Corrigenda", Journal of the American Statistical Association, 75: 765.

George, E. I. and McCulloch, R. E. (1993). "Variable Selection via Gibbs Sampling", Journal of the American Statistical Association, 88: 881-889. 
Hoerl, A. E. and Kennard, R. W. (1970a). "Ridge Regression: Biased Estimation for Nonorthogonal Problems", Technometrics, 12, 55-68.

Hoerl, A. E. and Kennard, R. W. (1970b). "Ridge Regression: Applications to Nonorthogonal Problems", Technometrics, 12, 69-82.

Hoeting, J. A., Madigan, D., Raftery, A. E. and Volinsky, C. T. (1999). "Bayesian Model Averaging: A Tutorial", Statistical Science, 14, 4, 382-417.

Leblanc, M. and Tibshirani, R. (1998). "Monotone Shrinkage of trees", Journal of Computational and Graphical Statistics, 7 (4), 417-433.

Levy, M. and Perng, S. K. (1984). "A Maximum Likelihood Prediction Function for de Linear Model with Consistency Results", Communications in Statistics, A-Theory and Methods, 13: 1257-1273.

Lindley, D. V. (1983). Lectures on Bayesian Statistics, Instituto de Matemática e Estatística da Universidade de São Paulo, 48p.

Mallows, C. L. (1973). "Some Comments on C C", Technometrics, 15, 661-675.

Mallows, C. L. (1995). "More Comments on C,", Technometrics, 37, 362-372.

Mitchell, T. J. and Beauchamp, J. J. (1988). "Bayesian Variable Selection in Linear Regression", Journal of the American Statistical Association, 83 (404): 1023-1036. 
Montgomery, D. C. and Peck, E. A. (1992). Introduction to Linear Regression Analysis, Second Edition. New York: John Wiley \& Sons. 527p.

Neter, J., Kutner, M. H., Nachtsheim, C. J., Wasserman, W. (1996). Applied Linear Statistical Models, Fourth Edition. Chicago: Irwin. 1408p.

Oishi, J. (1983). Regressão sobre cristas, Dissertação de Mestrado, IME-USP, 100p.

Rojas, M. D. I. (2000). Densidades Preditivas no Modelo de Regressão Linear, Dissertação de Mestrado, IME-USP, 122p.

S-Plus (2000). Pacote Estatístico Profissional - Release 3.

Tibshirani, R. (1996), "Regression Shrinkage and Selection via the "Lasso", Journal of the Royal Statistical Society B, 58, 267-288.

Zhang, P. (1993). "Model Selection via Multifold Cross-validation", The Annals of Statistics, 21, 299-313. 